\title{
Arithmetic Cryptography*
}

\author{
Benny Applebaum ${ }^{\dagger} \quad$ Jonathan Avron* $\quad$ Christina Brzuska ${ }^{\ddagger}$
}

Tuesday $14^{\text {th }}$ April, 2015

\begin{abstract}
We study the possibility of computing cryptographic primitives in a fully-black-box arithmetic model over a finite field $\mathbb{F}$. In this model, the input to a cryptographic primitive (e.g., encryption scheme) is given as a sequence of field elements, the honest parties are implemented by arithmetic circuits which make only a black-box use of the underlying field, and the adversary has a full (non-black-box) access to the field. This model captures many standard information-theoretic constructions.

We prove several positive and negative results in this model for various cryptographic tasks. On the positive side, we show that, under reasonable assumptions, computational primitives like commitment schemes, public-key encryption, oblivious transfer, and general secure two-party computation can be implemented in this model. On the negative side, we prove that garbled circuits, multiplicative-homomorphic encryption, and secure computation with low online complexity cannot be achieved in this model. Our results reveal a qualitative difference between the standard Boolean model and the arithmetic model, and explain, in retrospect, some of the limitations of previous constructions.
\end{abstract}

\footnotetext{
${ }^{*}$ A preliminary version of this paper has appeared in the 6th Innovations in Theoretical Computer Science (ITCS), 2015.

${ }^{\dagger}$ School of Electrical Engineering, Tel-Aviv University, \{bennyap@eng.tau.ac.il, avron@mail.tau.ac.il\}. Supported by ERC starting grant 639813 ERC-CLC, ISF grant 1155/11, Israel Ministry of Science and Technology grant 3-9094, GIF grant 1152/2011, and the Check Point Institute for Information Security.

${ }^{\ddagger}$ Microsoft Research Cambridge, UK, christina.brzuska@gmail.com. Work done while being a postdoctoral fellow at Tel Aviv university. Supported by Israel Ministry of Science and Technology grant 3-9094 and GIF grant $1152 / 2011$.
} 


\section{Contents}

1 Introduction 4

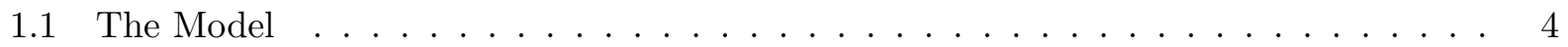

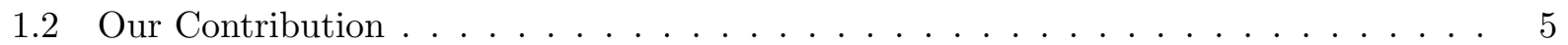

1.3 Discussion and Open Questions . . . . . . . . . . . . . . . . . 8

1.4 Previous Work $\ldots \ldots \ldots \ldots \ldots \ldots$

$\begin{array}{lll}2 & \text { Techniques } & 11\end{array}$

2.1 Negative Results . . . . . . . . . . . . . . . . . . . . . . . . . . 11

2.1 .1 Communication Lower Bounds in the PSM model . . . . . . . . . . . . 11

2.1 .2 Impossibility of Multiplicative Homomorphic Encryption. . . . . . . . . . . 13

2.2 Positive Results . . . . . . . . . . . . . . . . . . . . . . . . . . 14

$2.2 .1 \quad$ Arithmetic/Binary Symmetric Encryption $\ldots \ldots \ldots \ldots \ldots$

$2.2 .2 \quad$ Alternative approaches $\ldots \ldots \ldots \ldots \ldots \ldots \ldots \ldots$

$\begin{array}{llr}3 & \text { Preliminaries } & 16\end{array}$

3.1 Probabilistic Notation, Indistinguishability, and Entropy . . . . . . . . . . . . . 16

3.2 Polynomials and Rational Functions $\ldots \ldots \ldots \ldots$

3.3 Efficient Field Families . . . . . . . . . . . . . . . . . . . . . . . . . . 20

3.4 Arithmetic Circuits . . . . . . . . . . . . . . . . . . . . . 20

4 Cryptographic Primitives in the Arithmetic Setting 23

4.1 Pseudorandom Generators . . . . . . . . . . . . . . . . . . . . . . . . 23

4.2 Encryption Schemes $\ldots \ldots \ldots \ldots \ldots \ldots \ldots$

4.3 Commitments . . . . . . . . . . . . . . . . . . . . . . . . . 25

4.4 Randomized Encoding of Functions . . . . . . . . . . . . . . . . . . 26

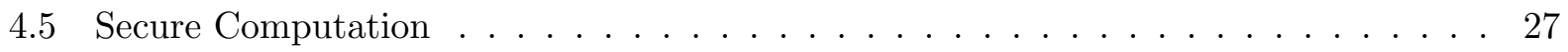

4.5 .1 The Semihonest Model . . . . . . . . . . . . . . . . . . . . . . . . . . 28

4.5 .2 The Malicious Model . . . . . . . . . . . . . . . . . . . . . . . . . . . . . . . . 29

\begin{tabular}{ll}
\hline I Lower Bounds & 30
\end{tabular}

5 Garbled Circuits and Randomized Encoding $\quad 30$

5.1 Proof of Theorem $5.1 \ldots \ldots \ldots \ldots \ldots \ldots$

5.2 Proof of Theorem $5.2 \ldots \ldots \ldots \ldots \ldots \ldots$

6 Arithmetic Multiparty Computation $\quad 35$

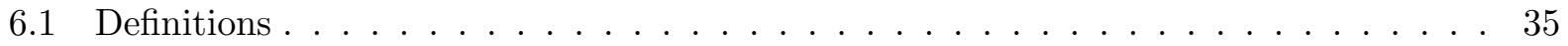

6.2 Main Result . . . . . . . . . . . . . . . . . . . . . . . . . . . . . . 36

$\begin{array}{lll}7 & \text { Homomorphic Encryption } & 39\end{array}$

7.1 Main Tool: Algorithm for the Arithmetic Predictability Problem . . . . . . . . . 39

7.2 Main Results: Attacking Strongly Homomorphic Encryption. . . . . . . . . . . . . . 41

7.3 Proof of Theorem 7.2 part $1 \ldots \ldots \ldots \ldots \ldots \ldots \ldots \ldots$ 
7.4 Proof of Theorem 7.2 part $2 \ldots \ldots \ldots \ldots \ldots$

7.4 .1 Removing random gates . . . . . . . . . . . . . . . . . 46

7.4 .2 Removing zerocheck gates . . . . . . . . . . . . . . . . . . . . 48

7.4 .3 Removing Division Gates . . . . . . . . . . . . . . . . . . . . . . 50

7.4.4 $\quad$ Putting It All Together (Proving Theorem 7.2 Part 2) . . . . . . . . . . . 50

\begin{tabular}{lll}
\hline II Positive Results & 51
\end{tabular}

8 The RLC Assumption $\quad 51$

9 Arithmetic Pseudorandom Generator $\quad 52$

9.1 Basic Observations . . . . . . . . . . . . . . . . . . . . . . . . . . . . . . . . . 52

9.2 Construction based on RLC $\ldots \ldots \ldots \ldots \ldots \ldots \ldots$

\begin{tabular}{ll}
\hline 0 Encryption & 54
\end{tabular}

10.1 One-Time Secure Arithmetic/Binary Encryption . . . . . . . . . . . . . . . 54

10.2 From ABE to Symmetric and Public-Key Encryption . . . . . . . . . . . . . . . 57

10.3 Direct Construction of Symmetric Encryption . . . . . . . . . . . . . . . 58

10.4 Direct Construction of Public Key Encryption . . . . . . . . . . . . . . . . . . 60

\begin{tabular}{ll}
\hline 1 Commitments & 64
\end{tabular}

11.1 Non-Interactive Statistically Binding String Commitment . . . . . . . . . . . . . 64

11.2 CRS Free Commitment Protocol $\ldots \ldots \ldots \ldots$

\begin{tabular}{|l|l}
\hline 2 Secure Computation & 67
\end{tabular}

$12.1\left(\begin{array}{l}2 \\ 1\end{array}\right)$-Arithmetic Oblivious Transfer . . . . . . . . . . . . . . . . . . . . 67

12.1 .1 Alternative Construction based Alekhnovich's PKE . . . . . . . . . . . . . 68

12.2 From $\left(\begin{array}{l}2 \\ 1\end{array}\right)$-AOT to Oblivious Linear Evaluation $\ldots \ldots \ldots \ldots$. . . . . . . . . . . . . . . . . . . . . .

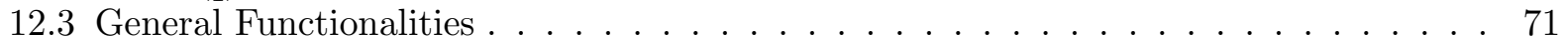

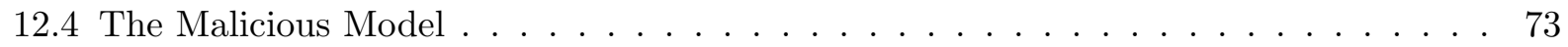

12.4.1 Proof of Theorem $12.16 \ldots \ldots \ldots \ldots \ldots$. . . . . . . . . . . . . . . . . . 


\section{Introduction}

This paper studies the possibility of solving cryptographic problems in a way which is independent from the underlying algebraic domain. We start by describing a concrete motivating example.

Consider the problem of computing over encrypted data where Alice wishes to store her private data $x=\left(x_{1}, \ldots, x_{n}\right)$ encrypted on a server while allowing the server to run some program $f$ on the data. Let us assume that each data item $x_{i}$ is taken from some large algebraic domain $\mathbb{F}$ (e.g., finite-precision reals) and, correspondingly, the program $f$ is described as a sequence of arithmetic operations over $\mathbb{F}$. Naturally, Alice would like to employ a fully homomorphic encryption (FHE) Gen09. However, standard FHE constructions typically assume that the data is represented as a binary string and the computation $f$ is represented by a Boolean circuit.

One way to solve the problem is to translate $x$ and $f$ to binary form. Unfortunately, this solution suffers from several limitations. First, such a translation is typically expensive as it introduces a large overhead (typically much larger than $\log |\mathbb{F}|)$.1 Secondly, such an emulation is not modular as it strongly depends on the bit-representation of $x$. Finally, in some scenarios Boolean emulation is simply not feasible since the parties do not have an access to the bit-wise representation of the field elements. For example, the data items $\left(x_{1}, \ldots, x_{n}\right)$ may be already "encrypted" under some algebraic scheme (e.g., given at the exponent of some group generator or represented by some graded encoding scheme [GGH13]).

A better solution would be to have an FHE that supports $\mathbb{F}$-operations. Striving for full generality, we would like to have an FHE that treats the field or ring $\mathbb{F}$ as an oracle which can be later instantiated with any concrete domain. In this paper we explore the feasibility of such schemes. More generally, we study the following basic question:

Which cryptographic primitives (if any) can be implemented independently of the underlying algebraic domain?

We formalize the above question via the following notion of arithmetic constructions of cryptographic primitives.

\section{$1.1 \quad$ The Model}

Cryptographic constructions. Standard cryptographic constructions can be typically described by a tuple of efficient (randomized) algorithms $P$ that implement the honest parties. The inputs to these algorithms consist of a binary string $x \in\{0,1\}^{*}$ (e.g., plaintext/ciphertext) and a security parameter $1^{\lambda}$ which, by default, is taken to be polynomial in the length of the input $x$. These algorithms should satisfy some syntactic properties (e.g., "correctness") as well as some security definition. We assume that the latter is formulated via a game between an adversary and a challenger. The construction is secure for a class of adversaries (e.g., polynomial-size Boolean circuits) if no adversary in the class can win the game with probability larger than some predefined threshold.

Arithmetic constructions. In our arithmetic model, the input $x=\left(x_{1}, \ldots, x_{n}\right)$ to the honest parties $P$ is a vector of generic field elements. The honest parties can manipulate field elements by applying field operations (addition, subtraction, multiplication, division, and zero-testing). There

\footnotetext{
${ }^{1}$ For example, for the case of finite fields with $n$-bit elements, the size of the best known Boolean multiplication circuits is $\omega(n \log n)$.
} 
is no other way to access the field elements. In particular, the honest parties do not have an access to the bit representation of the inputs or even to the size of $\mathbb{F}$. We also allow the honest parties to generate the field's constants 0 and 1, to sample random field elements, and to sample random bits. Overall, honest parties can be described by efficiently computable randomized arithmetic circuits. (See Section 3 for a formal definition.)

In contrast to the honest parties, the adversary is non-arithmetic and is captured, as usual, by some class of probabilistic Boolean circuits (e.g., uniform circuits of polynomial-size). Security should hold for any (adversarial) realization of $\mathbb{F}$. Formally, the standard security game is augmented with an additional preliminary step in which the adversary is allowed to specify a field by sending to the challenger a Boolean circuit which implements the field operations with respect to some (adversarially-chosen) binary representation. The game is continued as usual, where the adversary is now attacking the construction $P^{\mathbb{F}}$. Note that once $\mathbb{F}$ is specified, $P^{\mathbb{F}}$ can be written as a standard Boolean circuit. Hence security in the arithmetic setting guarantees that the construction $P^{\mathbb{F}}$ is secure for any concrete field oracle $\mathbb{F}$ which is realizable by our class of adversaries ${ }^{2}$

Example 1.1 (One-time encryption). To illustrate the model let us define an arithmetic perfectlysecure one-time encryption scheme. Syntactically, such a scheme consists of a key-generation algorithm KGen, encryption algorithm Enc, and decryption algorithm Dec which satisfy the perfect correctness condition:

$$
\underset{k \stackrel{R}{\leftarrow} \operatorname{KGen}_{n}}{\operatorname{Pr}}\left[\operatorname{Dec}_{k}\left(\operatorname{Enc}_{k}(m)\right)=m\right]=1, \quad \text { for every message } m \in \mathbb{F}^{n} .
$$

Perfect security can be defined via the following indistinguishability game: (1) For a security parameter $1^{n}$, the adversary specifies a field $\mathbb{F}$ and a pair of messages $m_{0}, m_{1} \in \mathbb{F}^{n}$; (2) The challenger responds with a ciphertext $c=\operatorname{Enc}_{k}\left(m_{b}\right)$ where $k \stackrel{R}{\leftarrow} \operatorname{KGen}_{n}$ and $b \stackrel{R}{\leftarrow}\{0,1\}$; (3) The adversary outputs a bit $b^{\prime}$ and wins the game if $b^{\prime}=b$. The scheme is perfectly-secure if no (computationallyunbounded) adversary can win the game with more than probability $\frac{1}{2}$.

A simple generalization of the well-known one-time pad gives rise to an arithmetic one-time encryption scheme. The key generation algorithm samples a random key $k \stackrel{R}{\leftarrow} \mathbb{F}^{n}$, to encrypt a message $m \in \mathbb{F}^{n}$ we output $m+k$ and to decrypt a ciphertext $c \in \mathbb{F}^{n}$ we output the message $c-k$. All the above operations can be implemented by randomized arithmetic circuits. It is not hard to see that the scheme is perfectly-secure. Namely, for any field $\mathbb{F}$ (or even group) chosen by a computationally-unbounded adversary, the winning probability cannot exceed $\frac{1}{2}$.

\subsection{Our Contribution}

Our goal in this paper is to find out which cryptographic primitives admit arithmetic constructions. We begin by observing that, similarly to the case of one-time pad, typical information-theoretic constructions naturally arithmetize. Notable examples include various secret sharing schemes Sha79, DF91, CF02], and classical information-theoretic secure multiparty protocols [BOGW88, CCD88].

\footnotetext{
${ }^{2}$ Note that the computational complexity of the field representation is limited by the computational power of the adversary. Specifically, if the primitive is secure against polynomial-size circuits then the underlying field must be implementable by a polynomial-size circuit. This limitation is inherent (for computationally-secure schemes), as otherwise, one can use an inefficient field representation to break the scheme (e.g., by embedding an NP-complete oracle).
} 
(See Section 1.4 for a detailed account of related works.) This raises the natural question of constructing computationally secure primitives in the arithmetic model. We give an affirmative answer to this question by providing arithmetic constructions of several computational primitives.

Informal Theorem 1.1. There are arithmetic constructions of public-key encryption, commitment scheme, oblivious linear evaluation (the arithmetic analog of oblivious transfer), and protocols for general secure multiparty computation without honest majority (e.g., two-party computation), assuming intractability assumptions related to linear codes.

We emphasize that our focus here is on feasibility rather than efficiency, and so we did not attempt to optimize the complexity of the constructions. The underlying intractability assumption essentially assumes the pseudorandomness of a matrix-vector pair $(M, \tilde{y})$ where $M$ is a random $m \times n$ generating matrix and $\tilde{y} \in \mathbb{F}^{m}$ is obtained by choosing a random codeword $y \in \operatorname{colSpan}(M)$ and replacing a random $p$-fraction of $y$ 's coordinates with random field elements ${ }^{3}$ This RandomLinear-Code assumption, which is denoted by $\operatorname{RLC}_{\mathbb{F}}(n, m, p)$, was previously put forward in [PS09]. If $\mathbb{F}$ is instantiated with the binary field, we get the standard Learning Parity with Noise (LPN) assumption GKL88, BFKL93. Indeed, some of the primitives in the above theorem can be constructed by extending various LPN-based schemes from the literature. (See Section 2.2.)

Theorem 1.1 shows that the arithmetic model is rich enough to allow highly non-trivial computational cryptography such as general secure two-party protocols. As a result, one may further hope to arithmetize all Boolean primitives. Our main results show that this is impossible. That is, we show that there are several cryptographic tasks which can be achieved in the standard model but cannot be implemented arithmetically. This include garbled circuits, secure computation protocols with "low" online communication, and multiplicative homomorphic encryption schemes. Details follow.

Garbled circuits. Yao's garbled circuit (GC) construction Yao86 maps any boolean circuit $C:\{0,1\}^{n} \rightarrow\{0,1\}^{m}$ together with secret randomness into a "garbled circuit" $\hat{C}$ along with $n$ "key" functions $K_{i}:\{0,1\} \rightarrow\{0,1\}^{k}$ such that, for any (unknown) input $x$, the garbled circuit $\hat{C}$ together with the $n$ keys $K_{x}=\left(K_{1}\left(x_{1}\right), \ldots, K_{n}\left(x_{n}\right)\right)$ reveal $C(x)$ but give no additional information about $x$. The latter requirement is formalized by requiring the existence of an efficient decoder which recovers $C(x)$ from $\left(\hat{C}, K_{x}\right)$ and an efficient simulator which, given $C(x)$, samples from a distribution which is computationally indistinguishable from $\left(\hat{C}, K_{x}\right)$. The keys are short in the sense that their length, $k$, depends only in the security parameter and does not grow with the input length or the size of $C$. Yao's celebrated result shows that such a transformation can be based on the existence of any pseudorandom generator [BM82, Yao82], or equivalently a one-way function [HILL99].

The definition of arithmetic garbled circuits naturally generalizes the Boolean setting. The target function $C: \mathbb{F}^{n} \rightarrow \mathbb{F}^{m}$ is now a formal polynomial (represented by an arithmetic circuit), and we would like to encode it into a garbled circuit $\hat{C}$, along with $n$ arithmetic key functions $K_{i}: \mathbb{F} \rightarrow \mathbb{F}^{k}$, such that $\hat{C}$ together with the $n$ outputs $K_{i}\left(x_{i}\right)$ reveal $C(x)$ and no additional information about $x$. As in the Boolean case, we require the existence of an arithmetic decoder

\footnotetext{
${ }^{3}$ This is contrasted with the more standard Learning-With-Errors (LWE) assumption Reg05] in which each coordinate of $y$ is perturbed with some "small" element from the ring $\mathbb{Z}_{p}$, e.g., drawn from the interval $\pm \alpha \cdot p$. Note that in the arithmetic setting it is unclear how to sample an element from an interval which grows with $p$, and so LWE constructions do not seem to arithmetize. See Section 1.3 for further discussion.
} 
and simulator. We say that the garbling is short if the key-length depends only in the security parameter (i.e., can be taken to be $n^{\varepsilon}$ for an arbitrary $\varepsilon>0$ ). A more relaxed notion is online efficiency, meaning that the key-length should be independent of the circuit complexity of $C$ but may grow with the input length. (The latter requirement is typically viewed as part of the definition of garbling schemes, cf. BHR12.)

The question of garbling arithmetic circuits has been open for a long time, and only recently some partial progress has been made [AIK11]. Still, so far there has been no fully arithmetic construction in which both the encoder and the decoder make a black-box use of $\mathbb{F}$. The next theorem shows that this is inherently impossible answering an open question from [Ish12.

Informal Theorem 1.2. There are no short arithmetic garbled circuits. Furthermore, assuming the existence of (standard) one-way functions, even online efficient arithmetic garbled circuits do not exist!

Recall that in the Boolean setting short garbled circuits can be constructed based on any oneway function, hence, Theorem 1.2 "separates" the Arithmetic model from the Boolean model. The proof of the theorem appears in Section 5.

Secure computation with low online complexity. Generalizing the above result, we prove a non-trivial lower-bound on the online communication complexity of semi-honest secure computation protocols. Roughly speaking, we allow the parties to exchange all the messages which solely depend on internal randomness at an "offline phase", and then move to an "online phase" in which the parties receive their inputs and may exchange messages based on their inputs (as well as their current view). Such an online/offline model was studied in several works [Bea95, IPS08, BDOZ11, DPSZ12, IKM $\left.{ }^{+} 13\right]$. In the standard Boolean setting, there are protocols which achieve highly efficient online communication complexity. For example, for efficient deterministic two-party functionalities $f:\{0,1\}^{n} \times\{0,1\}^{n} \rightarrow\{0,1\}^{m}$ which deliver the output to one of the parties (hereafter referred to as simple functionalities), one can obtain semi-honest protocols with online communication of $n^{1+\varepsilon}$ based on Yao's garbled circuit, or even $n+o(n)$ based on the succinct garbled circuit of [AIKW13]. In contrast, we show that in the arithmetic model the online communication complexity must grow with the complexity of the function.

Informal Theorem 1.3. Assume that arithmetic pseudorandom generators exist. Then, for every constant $c>0$ there exists a simple arithmetic two-party functionality $f: \mathbb{F}^{n} \times \mathbb{F}^{n} \rightarrow \mathbb{F}^{n^{c}}$ which cannot be securely computed by an arithmetic semi-honest protocol with online communication smaller than $\Omega\left(n^{c}\right)$ field elements.

The existence of an arithmetic pseudorandom generator follows from the RLC assumption. The theorem generalizes to the multiparty setting including the case of honest majority. (See Section 6.)

Multiplicative homomorphic encryption. A multiplicative homomorphic encryption scheme is a standard public-key encryption scheme in which, given only the public key, one can transform a ciphertext $c=\operatorname{Enc}_{\mathrm{pk}}(x)$ and a scalar $a \in \mathbb{F}$ (given in the clear) into a fresh encryption $c^{\prime}$ of the

\footnotetext{
${ }^{4}$ The theorem holds even if the simulator is allowed to be non-arithmetic or even inefficient. The latter case corresponds to an indistinguishability notion of security.
} 
product $a \cdot x$. Formally, we require an efficient (randomized) transformation $T$ such that, for every messages $x, a \in \mathbb{F}$ and almost all public keys pk, the distributions

$$
\left(c=\operatorname{Enc}_{\mathrm{pk}}(x), c^{\prime}=T(\mathrm{pk}, c, a)\right) \quad \text { and } \quad\left(c=\operatorname{Enc}_{\mathrm{pk}}(x), c^{\prime \prime}=\operatorname{Enc}_{\mathrm{pk}}(a \cdot x)\right)
$$

are statistically close. Two well known examples for such schemes (over different fields) are Goldwasser-Micali cryptosystem [GM84 and ElGamal cryptosystem [ElG84.

In Section 7 we show that multiplicative homomorphic encryption cannot be implemented arithmetically ${ }^{5}$

Informal Theorem 1.4. There are no multiplicative homomorphic encryption schemes.

In fact, the theorem holds even when the distributions in Eq. (1) are within small constant statistical distance (e.g., 1/6). Moreover, the theorem extends to the case of one-time secure private-key encryption schemes (and, under some additional conditions, to non-interactive perfectly binding commitments with multiplicative homomorphism). Interestingly, we can construct, in the arithmetic setting, (one-time secure) private-key encryption schemes which enjoy weak multiplicative homomorphism. Namely, only the marginals $c^{\prime}$ and $c^{\prime \prime}$, defined in (1), are identically distributed. So the main issue seems to be strong homomorphism, which cannot be achieved arithmetically, but can be easily achieved (for scalar multiplication) in the Boolean setting.

\subsection{Discussion and Open Questions}

Taken together, our positive and negative results suggest that the arithmetic model is highly nontrivial yet significantly weaker from the standard model. Beyond the natural interest in arithmetic constructions, our negative results explain, in retrospect, some of the limitations of previous results.

For example, AIK11] show that arithmetic garbled circuits can be constructed based on a special "key-shrinking" gadget, which can be viewed as a symmetric encryption over $\mathbb{F}$ with some homomorphic properties. They also provide an implementation of this gadget over the integers. This allows to garble circuits over the ring $\mathbb{Z}_{p}$ in a "semi-arithmetic" model, in which the encoder can treat the inputs as integers and the decoder is non-arithmetic. Theorem 1.2 shows that these limitations are inherent. Specifically, we can conclude that there are no arithmetic constructions of the key-shrinking gadget. Similarly, Theorem 1.3 partially explains the high online communication complexity of arithmetic MPC protocols such as the ones from [BOGW88, CCD88, CFIK03, IPS09.

Moreover, we believe that our results have interesting implications regarding the standard Boolean model. Inspired by computational complexity theory [BGS75, RR94, AW08], one can view our negative results as some form of a barrier.

The Arithmetization Barrier: If your construction "arithmetizes" then it faces the lower-bounds.

LPN/RLC vs. LWE. As an example, it seems that constructions which are based on the Learning-Parity-with-Noise assumption typically extend to the arithmetic setting under the RLC assumption. Therefore, "natural" LPN-based constructions are deemed to face our lower-bounds. Specifically, Theorem 1.4 suggests that it may be hard to design an LPN-based encryption with

\footnotetext{
${ }^{5}$ In the conference version of this paper, we stated a weaker impossibility result which applied only to restricted forms of arithmetic encryption schemes.
} 
(strong) multiplicative homomorphism. Since such schemes can be easily constructed under Regev's Learning-With-Errors (LWE) assumption [Reg05], this exposes a qualitative difference between the two assumptions. Indeed, this gap between strong LWE-type homomorphism (as in Eq. 1) which can be applied repeatedly, and weak LPN-type homomorphism which can be applied only a small number of times, seems to be crucial. This gap may also explain why LWE has so many powerful applications (e.g., fully homomorphic encryption [BV11]), while LPN is restricted to very basic primitives. The weak homomorphism supplied by typical LPN-based schemes was probably noticed by several researchers. The new insight, supplied by our arithmetic lower-bound, is that the lack of strong homomorphism is not just a limitation of a concrete construction, but it is, in fact, inherent to all arithmetic constructions. Quoting Pietrzak [Pie12] one may wonder: "... is there a fundamental reason why the more general LWE problem allows for such (rich cryptographic) objects, but LPN does not?" A simple answer would be: "LPN arithmetize but LWE doesn't."

IT constructions. Another example, for which the arithmetization barrier kicks in, is the case of information-theoretic (IT) constructions. Most of the standard techniques in this domain (e.g., polynomial-based error correcting codes) arithmetize, and so these constructions are deemed to be restricted by our lower-bounds. We mention that, in the area of IT-secure primitives, proving lower-bounds (even non-constructively) is notoriously hard 6 The arithmetic model restricts the honest parties, and as a result makes lower-bounds much more accessible while still capturing most existing schemes. We therefore view the arithmetic setting as a new promising starting point for proving lower-bounds for information-theoretic primitives.

From a more constructive perspective, instead of thinking of arithmetic lower-bounds as barriers, we may view them as road signs saying that in order to achieve some goals (e.g., basing homomorphic encryption on LPN), one must take a non-arithmetic route.

Open questions. We conclude with some open questions. First, there are several basic primitives whose existence in the arithmetic setting remains wide open. This includes Pseudorandom Functions, Collision Resistant Hash Functions, Message Authentication Codes, and Signatures. It will be also interesting to extend our positive results to a more restricted model which does not allow to sample random bits or to apply zero-testing. In fact, in this model we do not even know how to construct a one-way function based on a "standard assumption". On the negative side, one may ask whether our lower-bounds hold in a more liberal arithmetic model in which the parties are allowed to learn an upper-bound on the field size or to view a random representation of the field elements. (Such a model was considered by [IPS09], see Section 1.4.)

\subsection{Previous Work}

As already mentioned many information-theoretic primitives admit an arithmetic implementation. Notable examples include one-time MACs based on affine functions, Shamir's secret-sharing scheme [Sha79], the classical information-theoretic secure multiparty protocols of [BOGW88, CCD88. and the randomized encodings of [IK00]. Extensions of these results to generic black-box rings were given in [DF91, CF02, CFIK03].

\footnotetext{
${ }^{6} \mathrm{~A}$ classical example is the share size of secret-sharing schemes for general access structure. The situation becomes even more involved when it comes to more complicated objects such as secure multiparty protocols.
} 
Much less is known for computationally secure primitives. To the best of our knowledge, previous works only considered arithmetic models in which the honest parties have richer interface with the underlying field. (See below.) Therefore the resulting constructions do not satisfy our arithmetic notion.

The IPS model. Most relevant to our work is the model suggested by Ishai, Prabhakaran and Sahai [IPS09] (hereafter referred to as the IPS model) in the context of secure multiparty computation. In this model the parties are allowed to access the bit-representation of field elements, where the field and its representation are chosen by the adversary. This allows the honest parties to learn an upper-bound on the field size, and to feed field elements into a standard (Boolean) cryptographic scheme (e.g., encryption, or oblivious transfer). In contrast, such operations cannot be applied in our model $[7$ The work of Naor and Pinkas [NP99] yields semi-honest secure two-party protocols in the IPS model based on the pseudorandomness of noisy Reed-Solomon codewords. Ishai et al. [IPS09] extend this to the malicious model and to the case of general rings, and improve the efficiency and the underlying intractability assumptions. Both works rely on the existence of a Boolean Oblivious Transfer primitive.

Arithmetic reductions. Another line of works provides arithmetic constructions of high-level primitives $P$ (e.g., secure computation protocol) by making use of a lower-level primitive $Q$ (e.g., arithmetic oblivious-transfer) which is defined with respect to the field $\mathbb{F}$. This can be viewed as an arithmetic reduction from $P$ to $Q$. Arithmetic reductions from secure multiparty computation to Threshold Additive Homomorphic Encryption were given by [FH93] for the semi-honest model, and were extended by CDN01 to the malicious model (assuming that the underlying encryption is equipped with special-purpose zero-knowledge protocols). Similarly, the results of AIK11 can be viewed as an arithmetic reduction from garbling arithmetic circuits to the design of a special symmetric encryption over $\mathbb{F}$.

The Generic Group Model. It is instructive to compare our arithmetic model to the Generic Group Model (GGM) and its extensions [Sho97, MW98, Mau05, AM09]. The generic group model is an idealized model, where the adversary's computation is independent of the representation of the underlying cryptographic group (or ring). In contrast, in our model the honest players are arithmetic (independent of the field), while the adversary is non-arithmetic and has the power to specify the field and its representation. Correspondingly, these two models serve very different purposes: The GGM allows to prove unconditional hardness results against "generic attacks", while our model allows to increase the usability of cryptographic constructions by making them "field independent". Perhaps the best way to demonstrate the difference between the models is to see what happens when the ideal oracle is instantiated with a concrete field or ring. In our model, the resulting Boolean construction will remain secure by definition, whereas in the GGM the resulting scheme may become completely insecure [Den02].

\footnotetext{
${ }^{7}$ For example, in the IPS model a party can trivially commit to a field element $x \in \mathbb{F}$ by applying a binary commitment to the bit-representation of $x$. This is not possible in our model as $x$ can be manipulated only via the field operations.
} 


\section{Techniques}

\subsection{Negative Results}

At a high level, our main (negative) results are obtained by reducing the task of attacking arithmetic primitives to the task of "analyzing" arithmetic circuits. We solve the latter problem by making a novel use of tools (most notably partial derivatives) that were originally developed in the context of arithmetic complexity theory. Overall, our lower-bounds show that algorithms for arithmetic circuits can be used to attack arithmetic constructions. Below we give an outline of the proofs of the main negative results.

For ease of presentation, we sketch (in Section 2.1.1) a version of Theorems 1.2 and 1.3 in the Private Simultaneous Messages (PSM) model of [FKN94], which is conceptually simpler than garbled circuits and general secure computation protocols. Section 2.1 .2 contains an overview of the proof of Theorem 1.4 .

\subsubsection{Communication Lower Bounds in the PSM model}

The PSM model. Consider two parties Alice and Bob that have private inputs $x$ and $y$, respectively, and a shared random string $r$. Alice and Bob are each allowed to send a single message to a third party Carol, from which Carol is to learn the value of $f(x, y)$ for some predefined function $f$, but nothing else. The goal is to minimize the communication complexity. In the standard (Boolean) setting, one can use garbled circuits to obtain a protocol in which Alice's communication depends only on her input length and the security parameter $k$, and is independent of Bob's input length or the complexity of $f$. Specifically, under standard cryptographic assumptions, Alice's message $A(x ; r)$ can be of length $|x| \cdot k$ [FKN94], or even $|x|+k$ [AIKW13]. In contrast, we will prove that, in the arithmetic model, the length of Alice's message $A(x ; r)$ must grow with Bob's input.

Let Alice's input $x \in \mathbb{F}$ be a single field element, let Bob's input $y$ consist of two column vectors $y_{1}, y_{2} \in \mathbb{F}^{n}$, and let $f\left(x,\left(y_{1}, y_{2}\right)\right)=x \cdot y_{1}+y_{2}$ be the target function. We will show that if Alice's message is shorter than $n$, Carol can learn some non-trivial information about Bob's input. In patricular, Carol will output a non-zero vector which is orthogonal to $y_{1}$. (This clearly violates privacy as it allows Carol to exclude all but a $1 /|\mathbb{F}|$ fraction of all possible inputs for Bob.) Let us assume, for now, that the parties do not use division or zero-testing gates, and so all the parties are simply polynomials over $\mathbb{F}$.

We begin with a few observations. Fix the shared randomness $\mathbf{r}$, Bob's input $\mathbf{y}$, and Bob's message $\mathbf{b}=B(\mathbf{y} ; \mathbf{r})$, and consider the residual polynomials of Alice and Carol ${ }^{8}$ Alice computes a vector of univariate polynomials $A_{\mathbf{r}}(x): \mathbb{F} \rightarrow \mathbb{F}^{n-1}$ which takes her input $x \in \mathbb{F}$ and outputs a message $a \in \mathbb{F}^{n-1}$, and Carol computes a vector of multivariate polynomials $C_{\mathbf{b}}(a): \mathbb{F}^{n-1} \rightarrow \mathbb{F}^{n}$ which maps Alice's message $a \in \mathbb{F}^{n-1}$ to a vector of field elements $z \in \mathbb{F}^{n}$. By the correctness of the protocol, we have that

$$
f_{\mathbf{y}_{1}, \mathbf{y}_{\mathbf{2}}}(x)=C_{\mathbf{b}}\left(A_{\mathbf{r}}(x)\right), \quad \text { for every } x \in \mathbb{F},
$$

where $f_{\mathbf{y}_{1}, \mathbf{y}_{\mathbf{2}}}(x)=x \cdot \mathbf{y}_{\mathbf{1}}+\mathbf{y}_{\mathbf{2}}$. Let us fix a field $\mathbb{F}$ whose characteristic is larger than the degree of the polynomial $C_{\mathbf{b}}\left(A_{\mathbf{r}}(x)\right) 9$ Over such a large field, the univariate polynomial in the RHS of (2)

\footnotetext{
${ }^{8}$ We use bold fonts for fixed value, and standard fonts for non-fixed values which are treated as formal variables.

${ }^{9}$ Since the polynomial $C_{\mathbf{b}}\left(A_{\mathbf{r}}(x)\right)$ can be computed by a circuit of size $s=\operatorname{poly}(n)$, its degree is at most $2^{s}$ and so we can just use the field $\operatorname{GF}(p)$ where $p$ is a prime of bit length $2 s=\operatorname{poly}(n)$.
} 
and the univariate polynomial in the LHS are formally equivalent, namely, they represent the same polynomial in $\mathbb{F}[X]$. As a result, their formal partial derivatives are also equivalent:

$$
\partial f_{\mathbf{y}_{1}, \mathbf{y}_{\mathbf{2}}}(x) \equiv \partial C_{\mathbf{b}}\left(A_{\mathbf{r}}(x)\right) .
$$

By the definition of $f$ the LHS simplifies to $\mathbf{y}_{\mathbf{1}}$, and by applying the chain rule to the RHS we get

$$
\mathbf{y}_{\mathbf{1}} \equiv \mathcal{J} C_{\mathbf{b}}\left(A_{\mathbf{r}}(x)\right) \cdot \partial A_{\mathbf{r}}(x)
$$

Syntactically, $\partial A_{\mathbf{r}}(x)$ is a (column) vector of $n-1$ univariate polynomials that contains, for each output of $A_{\mathbf{r}}(x): \mathbb{F} \rightarrow \mathbb{F}^{n-1}$, the derivative with respect to the formal variable $x$. Similarly, the Jacobian matrix $\mathcal{J} C_{\mathbf{b}}(a): \mathbb{F}^{n-1} \rightarrow \mathbb{F}^{n \times n-1}$ is a matrix of multivariate polynomials whose $(i, j)$-th entry is the partial derivative of the $i$-th output of $C_{\mathbf{b}}(a): \mathbb{F}^{n-1} \rightarrow \mathbb{F}^{n}$ with respect to the $j$-th input (the formal variable $a_{j}$ ).

Let us now get back to Carol's attack. Carol does not know $\mathbf{r}$ and therefore she cannot compute neither $A_{\mathbf{r}}(x)$ nor its derivative $\partial A_{\mathbf{r}}(x)$. However, she knows $\mathbf{b}$ and therefore can compute a circuit for $C_{\mathbf{b}}$, which, by using standard techniques, can be transformed into a circuit for the Jacobian $\mathcal{J} C_{\mathbf{b}}$. Carol also received from Alice a message $\mathbf{a}=A_{\mathbf{r}}(\mathbf{x})$, where $\mathbf{x}$ is Alice's input, and so Carol can evaluate the circuit $\mathcal{J} C_{\mathbf{b}}$ at the point $\mathbf{a}$ and obtain the matrix $\mathbf{M}=\mathcal{J} C_{\mathbf{b}}(\mathbf{a}) \in \mathbb{F}^{n \times(n-1)}$. Now, the key observation is that

$$
\mathbf{y}_{\mathbf{1}}=\mathbf{M} \cdot \mathbf{v}, \quad \text { for some (unknown) vector } \mathbf{v} \text {. }
$$

Indeed, this follows by evaluating the RHS of (4) at the point $\mathbf{x}$ (and taking $\mathbf{v}=\partial A_{\mathbf{r}}(\mathbf{x})$ ). Overall, Carol now holds a matrix $\mathbf{M}$ whose columns span Bob's input $\mathbf{y}_{\mathbf{1}} \in \mathbb{F}^{n}$. Since $\mathbf{M}$ has only $n-1$ columns, Carol can find a non-zero vector which is orthogonal to $\mathbf{y}_{\mathbf{1}}$ and break the security of the protocol.

Handling zero-test gates. If the parties use zero-test gates then the functions computed by Alice and Carol are not polynomials anymore. As a result, (3) does not hold since the partial derivative of the function $P(x)=C_{\mathbf{b}}\left(A_{\mathbf{r}}(x)\right)$ is not defined. To solve the problem we show that it is possible to remove the zero-test gates. Assume, for simplicity, that the circuit $P(x)$ contains a single zero-test gate which is applied to the expression $Q(x)$. Note that $Q(x)$ is a polynomial of degree $d$ which is much smaller than the field. We distinguish between two cases: If $Q$ is the zero polynomial we remove the gate and replace its outcome with the constant 0 ; otherwise, we replace the gate with the constant 1 . This transformation changes the value of $P$ on at most $d$ points (the roots of $Q$ ), and therefore, the resulting polynomial $P^{\prime}$ agrees with the polynomial $f_{\mathbf{y}_{1}, \mathbf{y}_{2}}$ on all but $d$ points. Since both functions are low degree polynomials we conclude that they must be equal. The above argument easily generalizes to a large number of zero-test gates.

Some technicality arises due to the fact that the attacker Carol does not have an access to $P$, and can only compute its "outer part" $C_{\mathbf{b}}$. To see the problem, imagine that $C_{\mathbf{b}}$ contains a zero-check gate which is applied to a non-zero polynomial $Q$ which vanishes over the image of $A_{\mathbf{r}}$. In this case, the above procedure (applied to $C_{\mathbf{b}}$ alone) will fail miserably. We solve this issue by showing that, given a random point in the image of $A_{\mathbf{r}}$, one can remove the zero-test gates from $C_{\mathbf{b}}$ in a way which is consistent with the "inner part" $A_{\mathbf{r}}$. Since Carol can get such a point $a=A_{\mathbf{r}}(x)$ from Alice the attack goes through. The more general setting in which the parties may also use division gates is handled similarly (except for some minor technicalities). 
Extensions. The above argument shows that Alice's communication grows with the length of Bob's input. A stronger result would say that Alice's communication grows with the complexity of the function (even if Bob's input is also short). We can prove such a result via the use of a pseudorandom generator (PRG). Roughly speaking, we embed a PRG in the function $f$ such that a low communication protocol allows to break the pseudorandomness of the PRG. This approach extends to the setting of arithmetic garbled circuits and general secure multiparty protocols yielding Theorems 1.2 and 1.3 .

\subsubsection{Impossibility of Multiplicative Homomorphic Encryption}

Theorem 1.4 strongly relies on the existence of efficient algorithm for the following promise problem, denoted Arithmetic Predictability (AP): Given a pair of arithmetic circuits $P: \mathbb{F}^{n} \rightarrow \mathbb{F}^{m}$ and $T: \mathbb{F}^{n} \rightarrow \mathbb{F}$ distinguish between the case where

- (Predictable) For a randomly chosen $\boldsymbol{x} \stackrel{R}{\leftarrow} \mathbb{F}^{n}$, the random variable $T(\boldsymbol{x})$ is predictable given $P(\boldsymbol{x})$, i.e., there exits an efficient ${ }^{10}$ predictor which given $P(\boldsymbol{x})$ can guess, with high probability, the value of $T(\boldsymbol{x})$; and

- (Unpredictable) For a randomly chosen $\boldsymbol{x} \stackrel{R}{\leftarrow} \mathbb{F}^{n}$, the random variable $T(\boldsymbol{x})$ is (informationtheoretically) unpredictable given $P(\boldsymbol{x})$, i.e., any (computationally unbounded) predictor which gets to see $P(\boldsymbol{x})$, fails, with high probability, to guess the value of $T(\boldsymbol{x})$.

To prove Theorem 1.4 we show that attacking multiplicative homomorphic encryption reduces to solving AP. The idea is simple: given a public-key pk and a ciphertext $c=$ Enc $_{\mathrm{pk}}(b)$ of an unknown plaintext $b \in\{0,1\}$, we use the multiplicative homomorphism to construct the circuit $P(a)=f_{c, \text { pk }}(a)$ which maps a plaintext $a \in \mathbb{F}$ into a fresh encryption of $a \cdot b$. Consider the probability distribution of $P(\boldsymbol{a})$ induced by a uniform choice of $\boldsymbol{a} \stackrel{R}{\leftarrow} \mathbb{F}$ and the internal randomness of the homomorphic evaluator (here $c$ and pk are viewed as fixed constants). If $c$ is an encryption of 0 then $P(\boldsymbol{a})$ is simply a fresh encryption of the zero element, and $P$ loses all information regarding $\boldsymbol{a}$. As a result, $\boldsymbol{a}$ is unpredictable given $P(\boldsymbol{a})$. In contrast, if $c$ is an encryption of 1 then $P(\boldsymbol{a})$ is a fresh encryption of $\boldsymbol{a}$, and so, $\boldsymbol{a}$ can be predicted given $P(\boldsymbol{a})$ (e.g., by using the decryption algorithm). Hence, an efficient algorithm for predictability allows us to break the security of the multiplicative homomorphic encryption.

Building on the techniques of Dvir et al. [DGW07, we design an algorithm that solves AP in the case where the underlying field $\mathbb{F}$ is sufficiently large and the circuits $(P, T)$ compute polynomials (i.e., do not use division gates, zero-testing gates, and random bits). In fact, the algorithm in this case is surprisingly simple: Choose a random point $\boldsymbol{x} \stackrel{R}{\leftarrow} \mathbb{F}^{n}$ and check if the rows of the Jacobian $\mathcal{J} P(\boldsymbol{x}) \in \mathbb{F}^{m \times n}$ span the gradient $\partial T(\boldsymbol{x}) \in \mathbb{F}^{n}$ of the target polynomial $T(x)$. We further show that the case of a generalized arithmetic circuit $P$ with division gates, zero-testing gates, and random bits, reduces to the case where the circuit $P: \mathbb{F}^{n} \rightarrow \mathbb{F}^{m}$ computes polynomials. (The reduction introduces some "error terms" which force us to consider a more robust form of AP. Fortunately, the above algorithm generalizes to this setting as well. See Section 7)

\footnotetext{
${ }^{10}$ In fact, only the degree of $P$ should be upper-bounded (by $2^{\text {poly }(n)}$ ), while its circuit size may be arbitrary.
} 


\subsection{Positive Results}

Our positive results (Theorem 1.1) are based on three different approaches - outlined below.

\subsubsection{Arithmetic/Binary Symmetric Encryption}

One main approach is based on a new abstract notion of arithmetic/binary symmetric encryption (ABE). An ABE is an arithmetic symmetric encryption scheme which allows to encrypt a field element using a binary key. That is, while the scheme works in the arithmetic model, the key is essentially a string of bits given as a sequence of $0-1$ field elements. Such an encryption scheme allows us to import binary constructions to the arithmetic setting, and can be therefore viewed as a bridge between the binary world to the arithmetic world.

Given, for example, a standard binary public-key encryption scheme we obtain a new arithmetic public-key encryption by working in a hybrid mode. Namely, to encrypt a message $x \in \mathbb{F}$, encrypt $x$ via the ABE under a fresh private binary key $k$, and then use the binary public-key encryption to encrypt the binary message $k$. Conveniently, for this purpose it suffices to have a one-time secure ABE 11

Similarly to the case of public-key encryption, ABE can be used to obtain arithmetic constructions of CPA-secure symmetric-key encryption, and commitment schemes. In order to achieve arithmetic secure computation protocols, we will need an additional "weak homomorphism property": Given a ciphertext $E_{k}(x)$ and field elements $a, b \in \mathbb{F}$, it should be possible to generate a new ciphertext $c^{\prime}$ which decrypts to $a x+b$. (The new ciphertext $c^{\prime}$ does not have to look like a fresh ciphertext - hence the term "weak homomorphism" - and so this does not contradict our negative results.) For technical reasons, we also require a "simple" decryption algorithm (e.g., one that can be implemented by a polynomial-size arithmetic formula or branching program).

ABE based on RLC. We show that such a one-time secure ABE can be obtained under the (generalized) Random Linear Code assumption $\operatorname{RLC}_{\mathbb{F}}(n, m, p)$. To encrypt a message $x$, sample a random generating matrix $A \stackrel{R}{\leftarrow} \mathbb{F}^{m \times n}$ together with a random $p$-noisy codeword $y$, encode the message $x$ via a repetition code, and use the noisy codeword $y$ to mask the encoded message $x \cdot \mathbf{1}_{m}$. The resulting ciphertext consists of the pair $\left(A, y+x \cdot \mathbf{1}_{m}\right)$. The private-key is the set of all noisy coordinates, described as a binary vector. Decryption can be implemented by ignoring the noisy coordinates and solving a set of linear equations over $\mathbb{F}$. For properly chosen constants $m / n$ and $p$, the system will have a unique solution, with all but negligible probability.

From ABE to secure computation. Let us explain how to construct secure arithmetic twoparty computation from an ABE with weak homomorphism. The construction can be viewed as a variant of the construction of [IPS09]. Recall that a (binary) one-out-of-two oblivious transfer $\left(\left(\begin{array}{l}2 \\ 1\end{array}\right)\right.$-OT $)$ is a two-party functionality which takes two inputs $a_{0}, a_{1} \in\{0,1\}^{n}$ from a sender, and a selection bit $x \in\{0,1\}$ from the receiver and delivers to the receiver the value $a_{x}$.

We begin by converting a maliciously-secure binary $\left(\begin{array}{l}2 \\ 1\end{array}\right)$-OT into a maliciously-secure $\left(\begin{array}{l}2 \\ 1\end{array}\right)$ arithmetic oblivious transfer $\left(\left(\begin{array}{l}2 \\ 1\end{array}\right)\right.$-AOT) in which the sender's inputs $a_{0}, a_{1} \in \mathbb{F}$ are two field elements. The transformation uses an ABE in the natural way: The sender encrypts the arithmetic messages

\footnotetext{
${ }^{11}$ Although only one-time security is required, ABE cannot be achieved unconditionally as the message space (the size of $\mathbb{F}$ ) is larger than the key space which depends only on the security parameter and cannot grow with $\mathbb{F}$.
} 
$a_{0}$ and $a_{1}$ under binary keys $k_{0}, k_{1}$, and sends the ciphertexts to the receiver; then the receiver uses the binary $\left(\begin{array}{l}2 \\ 1\end{array}\right)$-OT to select one of two keys $k_{0}, k_{1}$.

Next, we convert $\left(\begin{array}{l}2 \\ 1\end{array}\right)$-AOT to Oblivious Linear Evaluation (OLE). The latter functionality takes two field elements $a, b \in \mathbb{F}$ from a sender, and another field element $x \in \mathbb{F}$ from the receiver and delivers to the receiver the value $a x+b$. The construction makes use of the ABE again, this time exploiting the weak homomorphism. Specifically, the receiver sends the ciphertext $c=E_{k}(x)$, and the sender uses the homomorphism to generate a ciphertext $c^{\prime}$ which decrypts to $a x+b$. This ciphertext cannot be sent back to the receiver as it leaks information on $a$ and $b$. Instead, a secure two-party computation protocol for decrypting $c^{\prime}$ is invoked. Since the input of the receiver is binary (and decryption has low-complexity), such a protocol can be implemented efficiently via a $\left(\begin{array}{l}2 \\ 1\end{array}\right)$-AOT (e.g., via the protocols of [CFIK03]) ${ }^{12}$ This gives a semi-honest OLE.

At this point, we can use the semi-honest OLE together with an arithmetic variant of the classical GMW protocol [GMW87] to obtain an arithmetic secure computation protocol for general arithmetic functions in the semi-honest model. This protocol can be transformed to the malicious setting using the IPS compiler [IPS08. To make the compiler work in our arithmetic setting, we need two additional tools: arithmetic multiparty protocol with security against a constant fraction of malicious parties (which can be constructed based on [BOGW88] or [CFIK03]), and a maliciously-secure $\left(\begin{array}{l}2 \\ 1\end{array}\right)$-AOT (which we already constructed).

\subsubsection{Alternative approaches}

Let us briefly mention two alternative approaches that can be used to derive arithmetic constructions for some of the primitives mentioned in Theorem 1.1 .

Arithmetizing LPN-based scheme. As already mentioned, existing LPN-based schemes easily extend to the arithmetic setting under the (generalized) Random Linear Code assumption. This gives alternative arithmetic constructions for primitives like symmetric encryption [GRS08, commitments AIK10, $\mathrm{KPC}^{+} 11$, and even public-key encryption Ale03 and $\left(\begin{array}{l}2 \\ 1\end{array}\right)$-AOT. This "direct approach" is inferior to the first (ABE-based) approach in terms of the strength of the underlying assumption. For example, using the direct approach, in order to obtain an arithmetic public-key encryption, we have to assume $\operatorname{RLC}(n, m, p)$ for constant-rate code $m=O(n)$ and sub-constant noise rate $p=O(1 / \sqrt{n})$. In the case of CPA-secure symmetric encryption, the direct approach requires hardness for any polynomial $m=m(n)$ and constant noise $p$. In contrast, for both primitives, the ABE-based approach requires only hardness for constant rate codes $m=O(n)$ and constant noise rate $p$. While all three assumptions are consistent with our knowledge, the third assumption is formally weaker than (i.e., implied by) the first two. Nevertheless, we also provide proofs based on the direct approach, as it is beneficial to demonstrate that known LPN-based schemes generalize to the arithmetic setting. (See discussion in Section 1.3.)

Arithmetizing Cryptographic Transformations. Another way to construct arithmetic primitives is to start with some concrete construction of a simple primitive $P$, and then use a standard (binary) cryptographic transformation from $P$ to a more complex primitive $Q$. For this, we have to translate the binary transformation to the arithmetic setting. Indeed, in some cases, existing binary transformations have a straightforward arithmetic analog. For example, we already mentioned

\footnotetext{
${ }^{12}$ For our concrete ABE, one can directly use $\left(\begin{array}{l}2 \\ 1\end{array}\right)$-AOT to securely deliver a re-randomized version of $c^{\prime}$.
} 
that the classical GMW construction [GMW87] of semi-honest secure computation from oblivious transfer (OT) naturally extends to the arithmetic setting [IPS09]. Similarly, we show that Naor's transform from PRGs to commitments has an arithmetic analog. This provides another arithmetic construction of commitments whose security can be reduced to the RLC assumption.

Interestingly, some binary cryptographic transforms do not seem to arithmetize. This typically happens if the construction inspects some input $x_{i} \in\{0,1\}$ and applies different operations depending on whether $x_{i}$ equals to zero or $x_{i}$ equals to one. This kind of arbitrary conditioning cannot be implemented in the arithmetic setting as $x_{i}$ varies over a huge (possibly exponential size) domain. As a typical example, consider the classical GGM construction [GGM86] of pseudorandom functions (PRFs) from pseudorandom generators (PRGs). In the GGM construction, the value of the PRF $F_{k}$ on a point $x \in\{0,1\}^{n}$ is computed by walking on an exponential size tree of lengthdoubling PRGs, where the $i$-th step is chosen based on the $i$-th bit of the input. It is not clear how to meaningfully adopt such a walk to the arithmetic case in which $x_{i} \in \mathbb{F}$. Similar "conditioning structure" appears in the Goldreich-Levin construction of hardcore predicates [GL89], and Yao's construction of garbled circuits from one-way functions. In fact, in the latter case our negative results show that finding an arithmetic analog of the binary construction is provably impossible. The problem of proving a similar negative result for the case of PRF, or, better yet, coming up with an arithmetic construction of a PRF, is left open for future research.

Organization. Following some preliminaries (Section 3) and definitions of arithmetic cryptographic primitives (Section 4), the main body of this work is divided into two parts. Part II is dedicated to lower bounds, and includes a section for each primitive: Randomized Encodings (Section 5), Secure Computation (Section 6) and Multiplicative Homomorphic Encryption (Section 7). The positive results appear in Part II] beginning with a presentation of the Random Linear Code intractability assumption (section 8), and proceeding with a section for each primitive: Pseudorandom Generators (Section 9), Encryption Schemes (Section 10), Commitments (Section 11) and Secure Computation Protocols (Section 12).

\section{Preliminaries}

In this section we provide some general preliminaries. We begin with standard background on probabilistic notation, indistinguishability, and entropy (Section 3.1), and basic facts about polynomials, rational functions and their derivatives (Section 3.2), and proceed with somewhat non-standard definitions of efficient field representations (Section 3.3), and generalized arithmetic circuits (Section 3.4).

\subsection{Probabilistic Notation, Indistinguishability, and Entropy}

Statistical distance and indistinguishability. A function $\mu(n)$ is said to be negligible in $n$ (denoted by neg $(n)$ ) if for any polynomial function $p(n)$ there exists $n^{\prime} \in \mathbb{N}$ such that $\mu(n) \leq 1 /|p(n)|$ for any $n>n^{\prime}$. Let $P$ and $Q$ be two distributions over the finite domain $\mathcal{U}$, we denote the statistical distance between $P$ and $Q$ by $\Delta(P, Q):=\frac{1}{2} \sum_{x \in \mathcal{U}}|\operatorname{Pr}[P(x)]-\operatorname{Pr}[Q(x)]|$. When $\Delta(P, Q)=0$ we

say the distributions are identically distributed and denote this by $P \stackrel{i}{\approx} Q$. We say that two distribution ensembles $\left\{P_{n}\right\}$ and $\left\{Q_{n}\right\}$ are statistically indistinguishable if $\Delta\left(P_{n}, Q_{n}\right)=\operatorname{neg}(n)$, we 
denote this this by $P_{n} \stackrel{s}{\approx} Q_{n}$. We say $P_{n}$ and $Q_{n}$ are computationally indistinguishable (denoted by $P_{n} \stackrel{c}{\approx} Q_{n}$ ) if for every polynomial-size family of circuits $13 \mathcal{A}=\left\{\mathcal{A}_{n}\right\}$, it holds that

$$
\left|\operatorname{Pr}_{x \leftarrow P_{n}}\left[\mathcal{A}_{n}\left(1^{n}, x\right)=1\right]-\operatorname{Pr}_{x \leftarrow Q_{n}}\left[\mathcal{A}_{n}\left(1^{n}, x\right)=1\right]\right|=\operatorname{neg}(n) .
$$

Entropy. For $p \in(0,1)$ and an integer $q>1$ we denote by $H_{q}(p)$ be the $q$-ary entropy function defined as $H_{q}(p):=-p \log _{q}(p)-(1-p) \log _{q}(1-p)$. The min-entropy $\mathbf{H}_{\infty}(X)$ of a random variable $X$ distributed over a finite domain, is defined as $\min _{x \in \operatorname{Supp}(X)} \log \left(\frac{1}{\operatorname{Pr}[X=x]}\right)$. For jointly distributed random variables $(X, Y)$, we define the predictability DORS08 of $Y$ given $X$, by $\operatorname{Pred}(Y \mid X)=$ $\max _{\mathcal{A}} \operatorname{Pr}[\mathcal{A}(X)=Y]$, where the maximum ranges over all possible (inefficient) algorithms $\mathcal{A}$. It is not hard to verify that the best guessing strategy for $\mathcal{A}(x)$ is to output the heaviest element $y$ in the conditional distribution $(Y \mid X=x)$, hence,

$$
\operatorname{Pred}(Y \mid X) \stackrel{\text { def }}{=} \underset{x \leftarrow ્ R}{\mathbb{E}}\left[\max _{y} \operatorname{Pr}[Y=y \mid X=x]\right]=\underset{x \leftarrow \mathbb{R}^{-}}{\mathbb{E}}\left[2^{-\mathbf{H}_{\infty}(Y \mid X=x)}\right] .
$$

A logarithmic version of predictability is captured by Average Min-Entropy [DORS08]:

$$
\tilde{\mathbf{H}}_{\infty}(Y \mid X) \stackrel{\text { def }}{=}-\log (\operatorname{Pred}(Y \mid X)) \text {. }
$$

We will need the following useful facts, which include (1) a variant of the Markov-inequality for average min-entropy, (2) the fact that applying a function to a random variable can only loose information, as well as (3) that conditioning on a random variables with $\lambda$ bits output can only reduce the average min-entropy by $\lambda$.

Fact 3.1. Let $W, X, Y$ be (possibly correlated) random variables. Then:

1. For any $\delta>0$, it holds that

$$
\underset{w \leftarrow \text { Pr }}{\operatorname{Pr}}\left[\tilde{\mathbf{H}}_{\infty}\left(Y_{W=w} \mid X_{W=w}\right)>\tilde{\mathbf{H}}_{\infty}(Y \mid X, W)-\log (1 / \delta)\right]>1-\delta,
$$

where $Y_{W=w}, X_{W=w}$ denote the joint distribution of $(X, Y)$ conditioned on the event $W=w$. In particular, $\operatorname{Pr}_{x \leftarrow X}\left[\mathbf{H}_{\infty}(Y \mid X=x)>\tilde{\mathbf{H}}_{\infty}(Y \mid X)-\log (1 / \delta)\right]>1-\delta$.

2. For every function $g$ it holds that $\tilde{\mathbf{H}}_{\infty}(Y \mid g(X)) \geq \tilde{\mathbf{H}}_{\infty}(Y \mid X)$. Furthermore, this holds even if $g$ is a randomized function which uses some internal random coins which are statistically independent of $Y$.

3. If $W$ has at most $2^{\lambda}$ possible values, then

$$
\tilde{\mathbf{H}}_{\infty}(Y \mid W, X) \geq \tilde{\mathbf{H}}_{\infty}((Y, W) \mid X)-\lambda \geq \tilde{\mathbf{H}}_{\infty}(Y \mid X)-\lambda .
$$

\footnotetext{
${ }^{13}$ We could use a uniform variant of indistinguishability, however, for our positive results (especially for secure computation) the non-uniform version is more natural and simplifies the treatment of auxiliary inputs. (The latter are necessary for composition theorems, cf. [Gol04, Chapter 7].)
} 
Proof. By definition,

$$
2^{-\tilde{\mathbf{H}}_{\infty}(Y \mid(X, W))}=\underset{w \leftarrow W}{\mathbb{R}}\left[\underset{2}{\mathbb{E}}\left[2^{-\mathbf{H}_{\infty}\left(Y_{W=w} \mid X_{W=w}\right)}\right],\right.
$$

hence, by Markov's inequality,

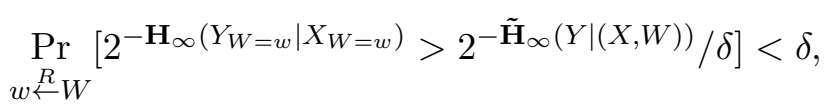

and the first item follows by taking logarithms.

To prove the second part note that if $\mathcal{A}$ predicts $Y$ given $g(X)$ with probability $p$, then we can define $\mathcal{A}^{\prime}$ which predicts $Y$ given $X$ with probability $p^{\prime} \geq p$ by letting $\mathcal{A}(x)=\mathcal{A}(g(x))$. Hence, $\operatorname{Pred}(Y \mid g(X)) \leq \operatorname{Pred}(Y \mid X)$. Finally, the third item is proved in [DORS08, Lemma 2.2.b].

\subsection{Polynomials and Rational Functions}

Notation. We let $\mathbb{F}$ denote a finite field. For a vector $\boldsymbol{x} \in \mathbb{F}^{n}$, we use the notation $|\boldsymbol{x}|$ to denote the number of elements in the vector $\boldsymbol{x}$. By $w(\boldsymbol{x})$ we denote the number of non-zero elements in $\boldsymbol{x}$. For a vector in $\mathbb{F}^{n}$ where all coordinates have the same value $\boldsymbol{x}$ we use the notation $\boldsymbol{x}^{n}$. We denote the inner product of two vectors $\boldsymbol{x}$ and $\boldsymbol{y}$ by $\boldsymbol{x} \cdot \boldsymbol{y}:=\sum_{i=1}^{n} \boldsymbol{x}_{i} \boldsymbol{y}_{i}$.

We will use bold fonts to emphasize the distinction between a formal variable $\mathrm{x}$ and its assignment on a point $\boldsymbol{x} \in \mathbb{F}$. A multivariate monomial $M(x)$ in variables $x=\left(x_{1}, \ldots, x_{n}\right)$ over a finite field $\mathbb{F}$ is defined as $M(x)=a x_{1}^{c_{1}} \cdots x_{n}^{c_{n}}$, where $a \in \mathbb{F}$ and $c_{i}$ are positive integers. A multivariate polynomial $P(x)$ is a sum of monomials. If $P(x)$ is a formal polynomial in $n$ variables $x=\left(x_{1}, \ldots, x_{n}\right)$, then it induces a function $P(x): \mathbb{F}^{n} \rightarrow \mathbb{F}$. A pair of polynomials $P(x)$ and $Q(x)$ are formally equivalent (denoted by $P \equiv Q$ ) if they compute the same formal polynomial, i.e., each monomial $M(x)$ appears in $P$ and $Q$ with the same coefficient. Clearly, if $P \equiv Q$ then the corresponding functions are also equal. The converse direction also holds as long as the degrees are smaller than the characteristic of the field.

A rational function $f$ is a quotient $v(x) / u(x)$ of two polynomials $v(x)$ and $u(x)$ where $u(x)$ is not the identically zero function. Note that $f$ is a partial function that is undefined at points $\boldsymbol{x}$ where $u(\boldsymbol{x})=0$. We say that a pair of rational functions $f(x)=f_{1}(x) / f_{2}(x)$ and $g(x)=g_{1}(x) / g_{2}(x)$ are equal (denoted $f=g$ ) if they agree on all inputs for which they are both defined. Note that this means that the polynomials $P(x)=f_{1}(x) g_{2}(x)$ and $Q(x)=f_{2}(x) g_{1}(x)$ compute the same function. The functions $f$ and $g$ are formally equivalent (denoted by $f \equiv g$ ) if the polynomials $P(x)$ and $Q(x)$ are formally equivalent.

Derivatives We now give the standard definitions of the formal derivative of multivariate polynomials over finite fields as well as some background, for a comprehensive treatment of derivatives over finite fields, see [SY10].

As opposed to a Euclidean space such as $\mathbb{R}$, in finite fields, there is no distance measure and thus, there is no notion of "limit" and "infinitely small numbers". Hence, instead of defining partial derivatives via limits as done for Euclidean spaces, over finite fields, derivative for polynomials and rational functions are a formal notion, that is, the derivative of $x^{n}$ is defined to be equal to $n x^{n-1}$ and this definition is extended to polynomials by additivity and to rational functions by using the quotient rule for derivatives. Throughout the paper, we will use formal derivatives only over sufficiently large fields whose characteristic is strictly larger than the degree of the underlying 
polynomial. It turns out that in this setting, formal derivatives inherit many of the properties of "standard derivatives" 14

Definition 3.2 (Partial Derivative). For a finite field $\mathbb{F}$ and monomial $M(x)=a x_{1}^{c_{1}} \cdots x_{n}^{c_{n}}$ with $a \in \mathbb{F}$ and for all $1 \leq i \leq n$, the (formal) partial derivative with respect to $x_{i}$ is defined as the monomial

$$
\partial_{x_{i}} M(x):=c_{i} a \cdot x_{1}^{c_{1}} \cdots x_{i}^{c_{i}-1} \cdots x_{n}^{c_{n}},
$$

where $c_{i}$ refers to $c_{i}$ times adding up the unit element of the field $\mathbb{F}$. The partial derivative of a polynomial is defined as the sum of the derivatives of its monomials. The partial derivative of a rational function $\frac{v(x)}{u(x)}$ is defined by

$$
\partial_{x_{i}}\left(\frac{v(x)}{u(x)}\right):=\frac{u(x) \partial_{x_{i}} v(x)-v(x) \partial_{x_{i}} u(x)}{(u(x))^{2}}
$$

Notice that the partial derivative of a polynomial (respectively rational function) is also a polynomial (respectively rational function). For $f(x)$ a vector of $\ell$ rational functions in $n$ variables $\left(f_{1}(x), \ldots, f_{\ell}(x)\right)$ we denote by $\partial_{x_{i}} f(x)$ the column vector $\left(\partial_{x_{i}} f_{1}(x), \ldots, \partial_{x_{i}} f_{\ell}(x)\right)^{T}$. In line with using normal font $x$ for variables and bold font $\boldsymbol{x}$ for a specific point, the notation $\partial_{x_{i}} f(\boldsymbol{x})$ refers to the partial derivative of $f(x)$ with respect to $x_{i}$ evaluated at the point $\boldsymbol{x}$.

Definition 3.3 (Formal Jacobian). For $f(x)=\left(f_{1}(x), \ldots, f_{\ell}(x)\right)$ a vector of $\ell$ rational functions in $n$ variables over a finite field $\mathbb{F}$, the Jacobian matrix $\mathcal{J} f(x)$ is the $\ell \times n$ matrix of formal derivatives $\left[\partial_{x_{1}} f(x), \ldots, \partial_{x_{n}} f(x)\right]$, that is, the $(i, j)$-th entry of $\mathcal{J} f(x)$ is $\partial_{x_{j}} f_{i}(x)$.

By $\mathcal{J} f(\boldsymbol{x})$ we denote all partial derivatives when evaluated at the same point $\boldsymbol{x}$. We denote the sub-matrix that contains only the columns of the subset $s \subseteq\{1, \ldots, n\}$ by $\mathcal{J}_{s} f(x)$. For the formal derivative of rational functions over finite fields, the product rule and the chain rule as commonly known, apply.

Fact 3.4 (Product Rule for Rational Functions). Let $f(x)$ and $g(x)$ be two rational functions in $n$ variables over a finite field $\mathbb{F}$. Then, for all $1 \leq i \leq n$, it holds that

$$
\partial_{x_{i}}(f(x) \cdot g(x))=g(x) \partial_{x_{i}} f(x)+f(x) \partial_{x_{i}} g(x) .
$$

Fact 3.5 (Chain Rule). Let $g(x)=\left(g_{1}(x), \ldots, g_{n}(x)\right)$ be a vector of rational functions in $n$ variables over a finite field $\mathbb{F}$, and let $f(y)$ be a rational function in $n$ variables over $\mathbb{F}$. Then, for all $1 \leq i \leq n$, it holds that

$$
\partial_{x_{i}} f(g(x))=\mathcal{J} f(g(x)) \cdot \partial_{x_{i}} g(x)
$$

Lemma 3.6 (Schwartz-Zippel [Sch80, Zip79]). Let $f\left(x_{1}, \ldots, x_{n}\right)$ be a non-zero polynomial of degree at most $d$ over a field $\mathbb{F}$ then the number of roots of $f$ is at most $d \cdot|\mathbb{F}|^{n-1}$ and the probability that $f\left(\boldsymbol{x}_{1}, \ldots, \boldsymbol{x}_{n}\right)=0$ for random $\boldsymbol{x}_{1}, \ldots, \boldsymbol{x}_{n}$ is smaller than $\frac{d}{|\mathbb{F}|}$.

\footnotetext{
${ }^{14}$ For example, in this case, a polynomial is constant if and only if its derivative is the all-zero function. This rule does not apply when the degree exceeds the field's characteristic, as demonstrated by the non-zero polynomial $x^{|\mathbb{F}|}$ whose formal derivative $|\mathbb{F}| x^{|\mathbb{F}|-1}$ is the zero function.
} 


\subsection{Efficient Field Families}

Throughout the paper, we consider finite fields whose elements have an efficient representation and whose field operations are efficiently computable. We begin by defining what it means for a Boolean circuit to implement a field.

Definition 3.7 (Circuit implementation of a field). Let $F$ be a Boolean circuit which takes as an input, an operation op $\in\{$ add, subtract, multiply, divide, constant, zerocheck, sample, bitsample $\}$ and up to two $\ell$ bits strings (using an appropriate encoding). $F$ is said to be a valid implementation of the finite field $\mathbb{F}$, if there is an injective mapping label : $\mathbb{F} \rightarrow\{0,1\}^{m}$ such that the following holds:

- For every command op $\in\{$ add, subtract, multiply, divide $\}$ and any $x, y \in \mathbb{F}$ it holds that $F($ op, label $(x)$, label $(y))=\operatorname{label}\left(x *_{\mathbb{F}} y\right)$ where $*_{\mathbb{F}}$ is the corresponding field operation.

- $F\left(\right.$ constant, $\left.0^{\ell}\right)=\operatorname{label}(0)$, and $F\left(\right.$ constant, $\left.1^{\ell}\right)=\operatorname{label}(1)$.

- If $a=\operatorname{label}(0)$, then $F$ (zerocheck, $a)=$ label(1).

- If $a=\operatorname{label}(x)$ for $x \in \mathbb{F}, x \neq 0$, then $F$ (zerocheck, $a)=\operatorname{label}(0)$.

- $F$ (sample) implements the uniform distribution over $\{$ label $(x): x \in \mathbb{F}\}$.

- $F$ (sample) implements the uniform distribution over $\{$ label $(x): x \in\{0,1\}\}$.

Definition 3.8 (Efficient field family). A polynomial-size Boolean circuit family $F=\left\{F_{n}\right\}$ implements a family of fields $\left\{\mathbb{F}_{n}\right\}$ if each circuit $F_{n}$ implements $\mathbb{F}_{n}$. In the uniform setting, we say that a PPT F implements a field if $F\left(1^{n}\right)$ outputs, with all but negligible probability, a circuit $F_{n}$ which implements some field (as per Definition 3.7). That is, $F\left(1^{n}\right)$ defines a probability distribution over finite fields.

We often speak of the family of (distributions over) fields $\left\{\mathbb{F}_{n}\right\}$ and its efficient implementation $F$ interchangeably. Moreover, we omit the subscript $n$ when it is clear from context. As an example of an efficient field family, for each sequence of $n$-bit primes $\left\{p_{n}\right\}_{n \in \mathbb{N}}$, we can implement the field family $\left\{\mathbb{F}_{n}=\operatorname{GF}\left(p_{n}\right)\right\}$ by a (non-uniform) Boolean circuit family $\left\{F_{n}\right\}$. The uniform definition captures, for example, a PPT $A$ which chooses a random $n$-bit prime $p$ and outputs a circuit which implements the field $\operatorname{GF}(p)$. These variants correspond to the standard distinction between uniform and non-uniform adversaries. We remark that all our results are not sensitive to uniformity issues. Specifically, our negative results hold even if the adversary is uniform, while our positive results hold both in the uniform and non-uniform adversarial model (depending on the underlying assumptions).

\subsection{Arithmetic Circuits}

An arithmetic circuit is a circuit whose wires carry field elements, and whose gates perform arithmetic operations. The circuit is black-box with respect to the field representation, i.e., it does not have access to the bit representation of the elements carried by its wires. In addition to the standard arithmetic operations, we allow "checking for zero". Although not an arithmetic operation, we find it hard to design meaningful schemes when even equality can not be recognized and so we allow for this operation in our model. 
Randomized arithmetic circuits also have two special, randomized gates: The sample gate drawns elements from the underlying field $\mathbb{F}$ uniformly at random, and the bitsample gate draws a uniformly random element from the set $\{0,1\}$ which contains the zero and one elements of the field.

Definition 3.9 (Arithmetic Circuit). An arithmetic circuit is a directed acyclic graph. Each input gate (source) is labeled by an input variable $x_{i}$, a constant 1 or 0 , or a randomized gate sample or bitsample. Internal Gates are labeled by:

\{add, subtract, multiply, divide, zerocheck $\}$

For an arithmetic circuit $C(x)$ with $n$ input variables and $m$ output variables any field $\mathbb{F}$ induces in the natural way a (randomized) mapping $C^{\mathbb{F}}(x): \mathbb{F}^{n} \rightarrow \mathbb{F}^{m}$. Furthermore a field implementation $F$ naturally induces a Boolean circuit $C^{F}(x)$ which implements the mapping $C^{\mathbb{F}}(x)$ with the representation $F$. We use $C^{F}(x), C^{\mathbb{F}}(x)$ and $C(x)$ interchangeably, when $F$ and $\mathbb{F}$ are clear from context.

Throughout this work when discussing a family of circuits $C=\left\{C_{n}\right\}$ we assume by default polynomial time uniformity. That is, there exist a PPT Turing machine that on input $1^{n}$ outputs a description of $C_{n}$.

We will occasionally consider restricted forms of arithmetic circuits that use only subset of the types of gates. Most notably, we will consider deterministic arithmetic circuits (which do not use sample, bitsample gates), deterministic arithmetic circuits without zerocheck gates (which compute rational functions) and deterministic arithmetic circuits without zerocheck, divide gates (which compute polynomials). We refer to the latter (most restricted) type of circuits as strictly arithmetic circuits. The following fact states that one can efficiently compute derivatives for deterministic arithmetic circuits without zerocheck gates.

Fact 3.10 (Efficient Differentiation [BS83]). Let $C(x)$ be a deterministic arithmetic circuit of size $s$ in $n$ variables without zerocheck gates, and let $f(x)$ be the corresponding rational function. Then for all $1 \leq i \leq n$, we can construct a circuit $C^{\prime}(x)$ which evaluates the partial derivative $\partial_{x_{i}} f(x)$ in time $O(s)$, and constructing $C^{\prime}(x)$ from $C(x)$ is a polynomial-time operation.

The derivative of a circuits with zerocheck gates is not well define. (In fact, such a circuit may not compute a rational function). Still, it turns out that, over large fields, such circuits can be "approximated" well by arithmetic circuits without zerocheck gates. Specifically, the following fact will be useful for our lower-bounds.

Proposition 3.11 (Removing zerocheck gates). Let $C: \mathbb{F}^{n} \rightarrow \mathbb{F}^{m}$ be deterministic arithmetic circuit with zerocheck gates of size $s$ which never divides by zero, and let $\mathbb{F}=\mathrm{GF}(p)$ be a primeorder field of cardinality larger than $(s+1) 2^{s+1}$. Then, there exists a deterministic arithmetic circuit $D: \mathbb{F}^{n} \rightarrow \mathbb{F}^{m}$ without zerocheck gates of size $s$ and a strictly arithmetic circuit $G: \mathbb{F}^{n} \rightarrow \mathbb{F}$ of size at most $s^{2}$ that computes a polynomial of degree $s 2^{s+1}$ which satisfy the following properties:

1. For every $\boldsymbol{x} \in \mathbb{F}^{n}$ which is not a root of $G$ it holds that $C(\boldsymbol{x})=D(\boldsymbol{x})$.

2. $D$ is obtained from $C$ be replacing the $i$-th zerocheck gate with some constant gate $b_{i} \in\{0,1\}$. Furthermore, the $i$-th zerocheck gate of $D$ evaluates to $b_{i}$ on all the inputs $\boldsymbol{x} \in \mathbb{F}^{n}$ which are not roots of $G$. 
3. If $C$ computes a polynomial then $D$ computes a rational function which is formally equivalent to $C$.

4. There exists a probabilistic algorithm that given $(C, p)$ outputs with probability $1-\beta$ the circuits $D$ and $G$ in time $\operatorname{poly}(s, \log p, \log (1 / \beta))$.

Proof. The circuit $D$ is defined by repeatedly applying the following subroutine: Choose the first zerocheck gate (according to some topological order) and consider the function $g$ computed by its incoming wire. If $g$ is the zero function replace the gate by the constant 1 ("the zero-test passes"); otherwise, replace the gate with the constant 0 ("the zero-test fails").

Observe that the function $g_{i}$ considered in the $i$-th iteration, is computed by a zerocheck-free circuit of size at most $s$, and therefore it is a rational function of the form $u_{i} / v_{i}$ where the degrees of the numerator $u_{i}$ and the denominator $v_{i}$ are bounded by $2^{s}$. Furthermore, observe that none of the $v_{i}$ is identically zero, since otherwise, the original circuit $C$ tries to divide by zero (when it is applied to some inputs). Using standard techniques (cf. [SY10, Proof of Thm. 2.11]) we can extract a strictly arithmetic circuit of size $s$ that computes $u_{i}$ (resp., $v_{i}$ ).

Call a point $\boldsymbol{x} \in \mathbb{F}^{n}$ bad if it is a root of some non-zero $u_{i}$ or some (non-zero) $v_{i}$, and call it good otherwise. Observe that $D$ and $C$ agree on all the good points. Moreover, the sequence of values that a good point $\boldsymbol{x}$ induces on the zerocheck gates of the original circuit $C$, corresponds exactly to the constants used by $D$ to replace these gates. Letting $G$ be the product of all the $u_{i}$ and $v_{i}$ we derive the first two items. Furthermore, the forth item follows by checking if each of the $u_{i}$ 's is identically zero via the standard Polynomial Identity Testing algorithm. (E.g., by evaluating $u_{i}$ on poly $(\log (s / \beta))$ random points and accepting if and only if all the outcomes equal to zero.)

It is left to prove the third item. Since the $u_{i}$ 's and $v_{i}$ 's are low-degree polynomials, we have, by the Schwartz-Zippel Lemma and by a union bound, that all but a $s 2^{s+1} /|\mathbb{F}|$ fraction of the inputs are good. Recall that $D$ and $C$ can be computed by a circuit of size $s$, and therefore the degrees of the polynomial $C$ and the numerator and denominator of $D=p / q$ are also upper-bounded by $2^{s}$. It is not hard to show that such low-degree functions which agree on so many (good) points must be formally equivalent. Indeed, if this is not the case, then the polynomial $C(x) q(x)-p(x)$ is a non-zero polynomial of degree $2^{s+1}$ with a fraction of $1-\left(s 2^{s+1} /|\mathbb{F}|\right)$ roots. Since our field is large $|\mathbb{F}|>(s+1) 2^{s+1}$, this contradicts the Schwartz-Zippel Lemma.

For some of our attacks, we will need the following variant of the zerocheck removal procedure.

Definition 3.12 (The algorithm $\mathcal{T}$ ). The algorithm $\mathcal{T}$ takes as input an arithmetic circuit $C$ : $\mathbb{F}^{n} \rightarrow \mathbb{F}^{m}$ defined over a prime field $\mathbb{F}$, and a point $\boldsymbol{x} \in \mathbb{F}^{n}$. The algorithm $\mathcal{T}$ evaluates the circuit $C$ on the point $\boldsymbol{x}$, and replaces each zerocheck gate $g$ with the constant 1 or the constant 0 depending on the value of that $\boldsymbol{x}$ induces on $g$. At the end, it outputs the resulting zerocheck-free circuit $C^{\prime}(x)$.

Imagine that the circuit $C=B \circ A$ consists of a composition of two arithmetic circuits an inner part $A$ and an outer part $B$. Further, assume that $\mathcal{T}$ is applied separately to $A$ and $B$, i.e., $A^{\prime}=\mathcal{T}(A, \boldsymbol{x})$ and $B^{\prime}=\mathcal{T}(B, A(\boldsymbol{x}))$. The following key lemma shows that, for a random $\boldsymbol{x}$ and sufficiently large $\mathbb{F}$, the composed circuit $B^{\prime} \circ A^{\prime}$ computes $f$, and, more importantly for our attacks, the chain rule applies to the corresponding derivatives.

Lemma 3.13. Let $f(x)$ be a strictly arithmetic circuit and let $A(x)$ and $B(y)$ be two deterministic arithmetic circuits such that $B(A(\boldsymbol{x}))$ agrees with the function $f(\boldsymbol{x})$ on every input $\boldsymbol{x} \in \mathbb{F}^{n}$ where 
$\mathbb{F}$ is a field of prime order $p>2^{2 s+1}$ and $s=\max$ size $\{f, B \circ A\}$. Then, for every $1 \leq i \leq n$

$$
\underset{\boldsymbol{x} \leftarrow \mathbb{F}^{n}}{\operatorname{Pr}}\left[\partial_{x_{i}} f(\boldsymbol{x})=\mathcal{J} B^{\prime}(A(\boldsymbol{x})) \cdot \partial_{x_{i}} A^{\prime}(\boldsymbol{x})\right] \geq 1-\frac{2 s 2^{s}}{|\mathbb{F}|},
$$

where $A^{\prime}:=\mathcal{T}(A, \boldsymbol{x})$, and $B^{\prime}:=\mathcal{T}(B, A(\boldsymbol{x}))$.

Proof. Let $C(x)=B(A(x))$ be the circuit obtained by composing $B$ on $A$. By Proposition 3.11 it holds that

$$
\underset{\boldsymbol{x} \leftarrow \mathbb{F}^{n}}{\operatorname{Pr}}[f \equiv \mathcal{T}(C, \boldsymbol{x})] \geq 1-\frac{2 s 2^{s}}{|\mathbb{F}|} .
$$

Fix some $\boldsymbol{x}$ for which the function $C^{\prime}=\mathcal{T}(C, \boldsymbol{x})$ is formally equivalent to $f$, and let $A^{\prime}=\mathcal{T}(A, \boldsymbol{x})$ and $B^{\prime}(B, A(\boldsymbol{x}))$. Observe that, by the definition of $\mathcal{T}$, the circuit $C^{\prime}$ can be written as $B^{\prime} \circ A^{\prime}$. Now, taking derivatives in Eq. (6) and applying the chain rule yields that for all $1 \leq i \leq n$

$$
\partial_{x_{i}} f(x) \equiv \mathcal{J} B^{\prime}\left(A^{\prime}(x)\right) \cdot \partial_{x_{i}} A^{\prime}(x)
$$

Fix some $1 \leq i \leq n$. Denote by $g(x)$ the polynomial which is computed by the LHS, and by $h(x)$ the rational function computed in the RHS. Our goal is to show that $g(\boldsymbol{x})=h(\boldsymbol{x})$ for our fixed $\boldsymbol{x}$. This boils down to showing that $h(\boldsymbol{x})$ is well-defined, i.e., the denominator of $h$ does not vanish in the point $\boldsymbol{x}$. To prove this note that: (1) $B^{\prime} \circ A^{\prime}$ is well defined on $\boldsymbol{x}$ (since $\mathcal{T}$ guarantees that $\left.B^{\prime}\left(A^{\prime}(\boldsymbol{x})\right)=f(\boldsymbol{x})\right) ;(2) h$ is the derivative of $B^{\prime} \circ A^{\prime}$; and (3) If a rational function $H$ is well-defined on a point $\boldsymbol{x}$ then its derivative $h$ is also well-defined on $\boldsymbol{x}$.

\section{Cryptographic Primitives in the Arithmetic Setting}

We define the arithmetic versions of the main cryptographic primitives studied in the work. The reader may want to skip this section for now and refer to it later when appropriate. In the following, we will let $n$ denote the security parameter.

\subsection{Pseudorandom Generators}

We begin with a definition of an arithmetic pseudorandom generator (APRG). Our definition of APRG requires the output to be pseudorandom over $\mathbb{F}^{\ell}$ but allows the input (the seed) to contain both random field elements and random bits as long their total number is $n$. We discuss this choice later in Section 9.

Definition 4.1 (Arithmetic Pseudorandom Generator). Let $A P R G=\left\{\mathrm{APRG}_{n}\right\}$ be a sequence of polynomial sized arithmetic circuits where $\mathrm{APRG}_{n}$ outputs a vector of $\ell(n)$ field elements and uses at most $n$ random gates (some of them may be for random field elements and some maybe be for sampling random bits) and no deterministic input gates. We say that APRG is an arithmetic pseudorandom generator (APRG) if it satisfies the following two properties:

1. Expansion: $\ell>n$. We refer to the ratio $\frac{\ell}{n}$ as the expansion factor and to the difference $\ell-n$ as the additive expansion.

2. Pseudorandomness: The winning probability of every efficient adversary $\mathcal{A}$ in the following pseudorandomness game is at most $1 / 2+\operatorname{neg}(n)$ : 
(a) Given a security parameter $1^{n}$, the adversary $\mathcal{A}$ picks a field implementation $\mathbb{F}$ and sends it to the challenger.

(b) The challenger samples $b \stackrel{R}{\leftarrow}\{0,1\}$.

$i$. If $b=0$ : the challenger sends to the adversary a sample $y \stackrel{R}{\leftarrow} \mathrm{APRG}_{n}$.

ii. If $b=1$ : the challenger sends to the adversary a sample $u \stackrel{R}{\leftarrow} \mathbb{F}^{\ell}$.

(c) The adversary outputs $b^{\prime}$ and wins if $b^{\prime}=b$.

An APRG is simple if it does not contain division or zerocheck gates (but may use random bits). We will sometimes view the APRG as a mapping from the seed s, i.e., randomness sampled by the random gates, to the output, denoted by APRG $(s)$. Note that the total length of $s$ is $n$ and it may consist random field elements and random bits.

\subsection{Encryption Schemes}

We now define the arithmetic version of an encryption scheme.

Definition 4.2 (Arithmetic Encryption Scheme). Let $\mathrm{KGen}=\left\{\mathrm{KGen}_{n}\right\}$, Enc $=\left\{\mathrm{Enc}_{n}\right\}$ and $\mathrm{Dec}=$ $\left\{\operatorname{Dec}_{n}\right\}$ be a uniform sequence of poly $(n)$ sized arithmetic circuits. (KGen, Enc, Dec) is an arithmetic public-key encryption scheme if:

1. Correctness: for every field $\mathbb{F}$, and for every message $x \in \mathbb{F}$

$$
\underset{(\mathrm{sk}, \mathrm{pk}) \stackrel{R}{\leftarrow} \mathrm{KGen}_{n}}{\operatorname{Pr}}\left[\operatorname{Dec}_{n}^{\mathbb{F}}\left(\operatorname{Enc}_{n}^{\mathbb{F}}(x, \mathrm{pk}), \mathrm{sk}\right)=x\right] \geq 1-\operatorname{neg}(n)
$$

where the probability is taken over the sampling of keys and the randomness of the circuits $\mathrm{Enc}_{n}^{\mathbb{F}}, \operatorname{Dec}_{n}^{\mathbb{F}}$.

2. Computational Security: For every efficient adversary $\mathcal{A}$ the wining probability in the following IND-CPA game is at most $1 / 2+\operatorname{neg}(n)$ :

IND-CPA-Game $\left(1^{n}\right)$ :

- The adversary receives $1^{n}$, chooses a field $\mathbb{F}$ and sends $\mathbb{F}$ to challenger.

- The challenger samples (pk, sk) $\stackrel{R}{\leftarrow} \mathrm{KGen}{ }_{n}^{\mathbb{F}}$ and passes pk to the adversary.

- (Chosen Plaintext queries:) Repeatedly do as long as adversary requests:

- The adversary sends some $x \in \mathbb{F}$.

- The challenger responds with $\operatorname{Enc}_{n}^{\mathbb{F}}(x, \mathrm{pk})$.

- The adversary sends some pair $x_{0}, x_{1} \in \mathbb{F}$.

- The challenger samples $b \stackrel{R}{\leftarrow}\{0,1\}$ and responds with $c \stackrel{R}{\leftarrow} \operatorname{Enc}_{n}^{\mathbb{F}}\left(x_{b}, \mathrm{pk}\right)$.

- The adversary outputs $b^{\prime}$ and wins if $b^{\prime}=b$.

In the symmetric-key setting $\mathrm{pk}=\mathrm{sk}$ and the challenger does not pass $\mathrm{pk}$ to the adversary. The scheme is one-time secure if it is secure against adversaries which make no chosen plaintext queries. An arithmetic symmetric encryption scheme with one-time security is called arithmetic/binary encryption $(A B E)$ if all the elements of the secret key sk are taken from the subset $\{0,1\} \subset \mathbb{F}$. That is, the key is essentially a string of bits given as a sequence of 0-1 Field elements. 
Remark 4.3 (Encrypting long messages). The above definition assumes that the message contains a single field element. One could naturally extend the definition to support longer vectors (either with fixed block-length $\ell$ or with unbounded block length). We note that, as in the binary setting, a CPA-secure construction that supports a single message can be easily extended to encrypt a sequence of field elements by encrypting each element separately each time with fresh randomness (cf. [KL08, Section 3.4]).

\subsection{Commitments}

We consider non-interactive commitments. Such schemes are parameterized by a public key pk which is chosen by some trusted party or given as part of a Common Reference String (CRS).

Definition 4.4 (Statistically Binding Commitment Scheme). Let KGen $=\left\{\mathrm{KGen}_{n}\right\}$, Com $=$ $\left\{\mathrm{Com}_{n}\right\}$ and $\mathrm{Ver}=\left\{\mathrm{Ver}_{n}\right\}$ be a uniform sequence of polynomial sized arithmetic circuits. We denote the output of the circuit $\operatorname{Com}_{n}$ by $(c, d)$, where $c$ is the commitment and $d$ the decommitment. We say (KGen, Com, Ver) are a statistically binding commitment scheme if:

1. Correctness: For every field $\mathbb{F}$ and for every message $x \in \mathbb{F}$ it holds:

$$
\underset{\mathrm{pk} \stackrel{R}{\leftarrow} \mathrm{KGen}_{n}^{\mathbb{P}}}{\operatorname{Pr}}\left[\operatorname{Ver}_{n}^{\mathbb{F}}\left(\mathrm{pk}, x, \operatorname{Com}_{n}^{\mathbb{F}}(x, \mathrm{pk})\right)\right] \geq 1-\operatorname{neg}(n)
$$

where the probability is taken over the randomness of the circuits $\operatorname{Com}_{n}^{\mathbb{F}}$ and $\operatorname{Ver}_{n}^{\mathbb{F}}$.

2. Statistically Binding: For every field $\mathbb{F}$, with overwhelming probability over the choice of $\mathrm{pk} \stackrel{R}{\leftarrow} \mathrm{KGen}_{n}^{\mathbb{F}}$, no commitment $c$ can be opened in two different ways, i.e.,

$$
\left(\operatorname{Ver}_{n}^{\mathbb{F}}(\mathrm{pk}, x, c, d)=1\right) \wedge\left(\operatorname{Ver}_{n}^{\mathbb{F}}\left(\mathrm{pk}, x^{\prime}, c, d\right)=1\right) \Rightarrow x=x^{\prime}
$$

3. Computationally Hiding: No efficient adversary $\mathcal{A}$ can win the following game with probability greater than $1 / 2+\operatorname{neg}(n)$.

\section{IND-CPA Game $\left(1^{n}\right)$ :}

- $\mathcal{A}$ receives $1^{n}$, chooses a field $\mathbb{F}$ and sends $\mathbb{F}$ to the challenger.

- The challenger samples $\mathrm{pk} \stackrel{R}{\leftarrow} \mathrm{KGen}_{n}^{\mathbb{F}}$ and passes pk to the adversary.

- The adversary chooses $x_{0}, x_{1} \in \mathbb{F}$.

- The challenger samples $b \in\{0,1\}$, computes $(c, d) \stackrel{R}{\leftarrow} \operatorname{Com}_{n}^{\mathbb{F}}\left(x_{b}, \mathrm{pk}\right)$ and sends $c$.

- The adversary outputs $b^{\prime}$ and wins if $b^{\prime}=b$.

Without loss of generality, we assume that the de-committment string d consists of the internal randomness of the committer (which is a sequence of field elements and zero-one values). Under this convention, we let the output of $\mathrm{Com}_{n}^{\mathbb{F}}$ denote only the commitment string c.

Remark 4.5 (CRS-free variant). We will also consider a CRS-free variant of commitments. Syntactically, we still consider a non-interactive commitment function parameterized by a public-key which is generated by a key-generation algorithm. However, the hiding property should hold for every (adversarially chosen) public-key. This gives rise to a two-message commitment protocol in the standard model (no CRS) in which the receiver chooses the public-key pk. 


\subsection{Randomized Encoding of Functions}

We formalize arithmetic garbled circuits via the framework of randomized encoding of functions [IK00, AIK04. Roughly speaking, a deterministic function $f$ is encoded by a randomized arithmetic function $\hat{f}$ if for every input $x$ the distribution $\hat{f}(x)$, induced by the internal randomness of $\hat{f}$, reveals the value of $f(x)$ and nothing else.

Definition 4.6 (Arithmetic Randomized Encoding). Let $f=\left\{f_{n}\right\}$ be a family of polynomialsized strictly arithmetic circuits where $f_{n}$ has $n$ inputs. A family of polynomial-sized randomized arithmetic circuits $\hat{f}=\left\{\hat{f}_{n}\right\}$ and a family of polynomial-sized deterministic arithmetic circuits $\mathrm{Dec}=\left\{\operatorname{Dec}_{n}\right\}$ form a Randomized Encoding of $f$ if:

1. Perfect Correctness: for every field $\mathbb{F}$, for every $n \in \mathbb{N}$ and every $\boldsymbol{x} \in \mathbb{F}^{n}$

$$
\operatorname{Pr}\left[\operatorname{Dec}_{n}^{\mathbb{F}}\left(\hat{f}_{n}^{\mathbb{F}}(\boldsymbol{x})\right)=f_{n}^{\mathbb{F}}(\boldsymbol{x})\right]=1,
$$

where the probability is taken over the randomness of the encoding circuit $\hat{f}_{n}^{\mathbb{F}}$.

2. Computational Security (Indistinguishability):

For any PPT $\mathcal{A}\left(1^{n}\right)$ the winning probability in the following game is at most $1 / 2+\operatorname{neg}(n)$ :

- The adversary $\mathcal{A}$ chooses an implementation of a field $\mathbb{F}$ and $\boldsymbol{x}_{0}, \boldsymbol{x}_{1} \in \mathbb{F}^{n}$ s.t. $f_{n}^{\mathbb{F}}\left(\boldsymbol{x}_{0}\right)=$ $f_{n}^{\mathbb{F}}\left(\boldsymbol{x}_{1}\right)$ and sends to the challenger $\mathbb{F}, \boldsymbol{x}_{0}, \boldsymbol{x}_{1}$.

- The challenger samples $b \stackrel{R}{\leftarrow}\{0,1\}$ and $c=\hat{f}_{n}^{\mathbb{F}}\left(\boldsymbol{x}_{b}\right)$ and sends $c$ to $\mathcal{A}$.

- The adversary $\mathcal{A}$ outputs a bit $b^{\prime}$ and wins if $b^{\prime}=b$.

Remark 4.7. We mention that security is typically formalized via a stronger simulation-based definition. However, since we will be proving lower-bounds, a weaker security definition only makes our results stronger. Also, note that we restrict the encoded function $f$ to be computed by a strictly arithmetic circuit (i.e., by an arithmetic circuit over addition and multiplication gates only), whereas the encoding $\hat{f}$ is allowed to be an arbitrary arithmetic circuit. Again, this only makes our lower-bounds stronger.

Without any efficiency restriction on the encoder $\hat{f}$, the notion of randomized encoding becomes trivial (simply take $\hat{f}=f$ ). Below we present several common syntactic and efficiency requirements which are motivated by different RE applications.

Decomposable encoding. For many applications it makes sense to distinguish between the online part, the part of the output of $\hat{f}$ that depends on the input vector $x$, and the offline part, the part of the output that depends only on the randomness $r$ of the encoding. We will emphasize this distinction by splitting $\hat{f}(x ; r)$ into the functions $\hat{x}(x ; r)$ and $\hat{r}(r)$, where $r$ denotes the outcome of the random gates. A randomized encoding $(\hat{f}, \mathrm{Dec})$ is fully decomposable if each of the outputs of the encoder $\hat{f}(x ; r)$ depends on at most a single element in the input vector $x$. In such a case we will write $\hat{x}(x ; r)$ as $\left(\hat{x}_{1}\left(x_{1} ; r\right), \ldots, \hat{x}_{n}\left(x_{n} ; r\right)\right)$ to emphasize which part of the encoding depends on which input. One may define a weaker form of decomposability. Suppose that the encoded function $f$ is defined over two input vectors $x$ and $y$, then an encoding ( $\hat{f}$, Dec) is 2-decomposable if each output of $\hat{f}(x, y ; r)$ depends either on $x$ or on $y$ but not on both (but may depend arbitrarily on 


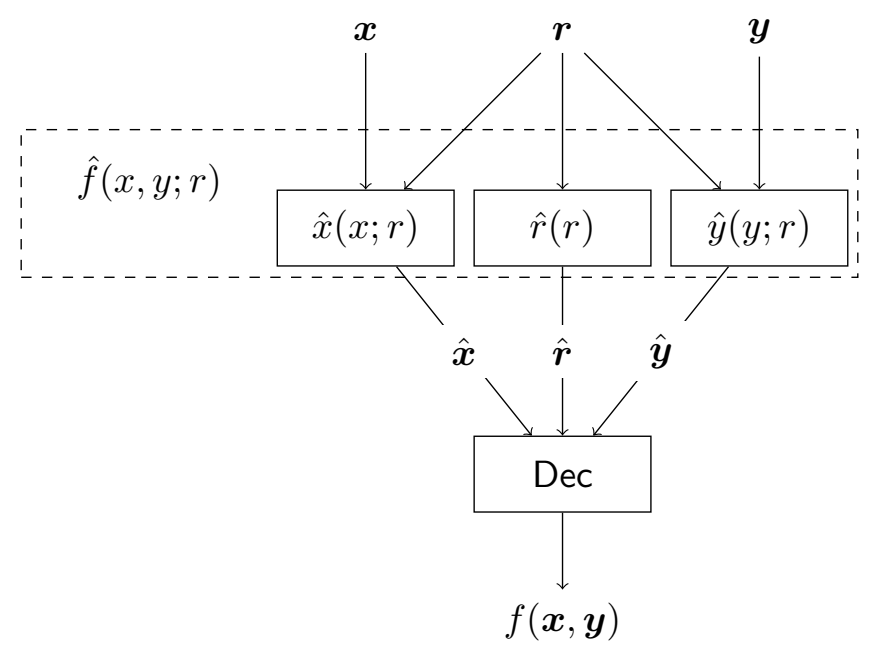

Figure 1: 2-Decomposable Randomized Encoding

$r)$. In this case we will usually denote $\hat{f}(x, y ; r)=(\hat{x}(x ; r), \hat{y}(y ; r), \hat{r}(r))$, to clarify which part of the encoding depends on which input. This notion corresponds to the PSM model described at the introduction 15

\subsection{Secure Computation}

Arithmetic functionalities. A two party arithmetic functionality $f: \mathbb{F}^{*} \times \mathbb{F}^{*} \rightarrow \mathbb{F}^{*} \times \mathbb{F}^{*}$ is described by a sequence of polynomial-size arithmetic circuits $\left\{f_{n}\right\}$ where each input and output gates are labeled by $A$ (for Alice) or by $B$ (for Bob). For simplicity, we will consider only deterministic functionalities and always assume that in $f_{n}$ both Alice and Bob have exactly $n$ inputs. Both assumptions hold without loss of generality. (See Gol04] Proposition 7.3.4. and Section 7.2.1.1.) For a field implementation $\mathbb{F}$ and pair of inputs $x, y \in \mathbb{F}^{n}$ for Alice and Bob, we let $f_{1}^{\mathbb{F}}(x, y)$ (resp., $\left.f_{2}^{\mathbb{F}}(x, y)\right)$ denote the outputs of Alice (resp., Bob).

Arithmetic Protocols A two-party arithmetic protocol $\Pi$ is a pair of (uniform) polynomial size arithmetic circuits $A=\left\{A_{n}\right\}$ and $B=\left\{B_{n}\right\}$, indexed by input length $n$. The circuits $A_{n}$ and $B_{n}$ interact via special "interaction gates" Send and Receive. Send gates have unbounded fan-in and no fan-out and Receive gates have an unbounded fan-out and zero fan-in. These gates are assumed to be topologically ordered from 1 to $n(n)$. We assume that the gates are aligned so that in $A_{n}$, odd-numbered interaction gates are all Send gates and even-numbered interaction gates are Receive gates, and, vise versa for $B_{n}$. Semantically, we assume that the $i$-th Send gate of a party (say $A$ ) corresponds to the $i$-th Receive gate of the other party, i.e., if $x \in \mathbb{F}^{*}$ is the input to Send then Receive $=x$. For every field implementation $\mathbb{F}_{n}$, the protocol naturally defines a joint computation which maps a pair of inputs $(x, y) \in \mathbb{F}_{n}^{n} \times \mathbb{F}_{n}^{n}$, to a pair of outputs Output ${ }^{\Pi, \mathbb{F}_{n}}(x, y)=$ (Output $_{1}$, Output $_{2}$ ) where Output 1 is the output of $A_{n}$ and Output ${ }_{2}$ is the output of $B_{n}$. The view

\footnotetext{
${ }^{15}$ To see this, let $r$ be the randomness shared between Alice and Bob, and let Alice (resp., Bob) send $\hat{x}(x ; r)$ (resp., $\hat{y}(y ; r))$ to Carol. In addition, the value $\hat{r}(r)$ can be sent to Carol in an offline phase or by one of the parties. Given all these values, Carol can learns $f(x, y)$ (using the decoder) without learning anything else.
} 
of $A$ during an execution of $\Pi$ over $\mathbb{F}_{n}$ on inputs $x, y \in \mathbb{F}^{n}$, denoted by $\operatorname{View}_{1}^{\Pi, \mathbb{F}}(x, y)$, is the random variable which consists of the deterministic and random inputs of $A_{n}$ and all the messages sent by $B_{n}$. The view of $B$ is defined similarly, and is denoted by $\operatorname{View}_{2}^{\Pi, \mathbb{F}}(x, y)$.

\subsubsection{The Semihonest Model}

Definition 4.8 (Semi-Honest Secure Computation (Deterministic Functionalities)). We say that a two party arithmetic protocol $\Pi=\left(\left\{A_{n}\right\},\left\{B_{n}\right\}\right)$ privately computes a (deterministic) functionality $f: \mathbb{F}^{*} \times \mathbb{F}^{*} \rightarrow \mathbb{F}^{*} \times \mathbb{F}^{*}$ if the following hold for every efficient field family $\left\{\mathbb{F}_{n}\right\}$ :

- Correctness: For every $x, y \in \mathbb{F}_{n}^{n}$,

$$
\operatorname{Pr}\left[\text { Output }^{\Pi, \mathbb{F}_{n}}(x, y) \neq f^{\mathbb{F}_{n}}(x, y)\right] \leq \operatorname{neg}(n) .
$$

- Privacy: There exist probabilistic polynomial time algorithms $\operatorname{Sim}_{1}$ and $\operatorname{Sim}_{2}$ such that

$$
\left\{\operatorname{View}_{1}^{\Pi, \mathbb{F}_{n}}(x, y)\right\}_{x, y \in \mathbb{F}_{n}^{n}} \stackrel{c}{\approx}\left\{\operatorname{Sim}_{1}\left(x, f_{1}^{\mathbb{F}_{n}}(x, y)\right)\right\}_{x, y \in \mathbb{F}_{n}^{n}}
$$

and

$$
\left\{\operatorname{View}_{2}^{\Pi, \mathbb{F}_{n}}(x, y)\right\}_{x, y \in \mathbb{F}_{n}^{n}} \stackrel{c}{\approx}\left\{\operatorname{Sim}_{2}\left(y, f_{2}^{\mathbb{F}_{n}}(x, y)\right)\right\}_{x, y \in \mathbb{F}_{n}^{n}}
$$

Note that the definition allows the simulators to depend on the field family $\left\{\mathbb{F}_{n}\right\}$ in a non-blackbox way. Nevertheless, in all our constructions the simulators will also be defined by arithmetic circuits and will access the underlying field $\mathbb{F}$ only in a black-box way. For our lower-bounds, we will consider a weaker version of One-Sided Semi-Honest Security (see Section 6 for details).

Partial functionalities, finite functionalities, and different input lengths. We sometimes consider partial functionalities (e.g., when some of the inputs are binary strings) in this case the above requirements should be adopted by indexing the ensembles by appropriate inputs. Furthermore, we will use finite functionalities (e.g., oblivious transfer) and in this case incorporate the security parameter $1^{n}$ as part of the parties input. We will also consider functionalities in which the input length of Alice may be different from the input length of Bob, with the implicit convention that we can always guarantee equal input length by padding.

Privacy reductions. To define secure reductions, consider the following hybrid model. A twoparty protocol augmented with an oracle to the two-party arithmetic functionality $g$ is a standard protocol in which the parties are allowed to invoke $g$, i.e., a trusted party to which they can securely send inputs and receive the corresponding outputs. Formally, the circuits $A_{n}$ and $B_{n}$ are equipped with an equal number of special $g$ gates, where the $i$-th $g$-gate takes an input $a_{i}$ from $A$ and an input $b_{i}$ from $B$, and returns an output $g_{1}(a, b)$ to $A$ and $g_{2}(a, b)$ to $B$. When $A_{n}$ and $B_{n}$ are instantiated with a field $\mathbb{F}$, the functionality $g$ is instantiated with the same field $\mathbb{F}$ as well. The notion of private computation (Definition 4.8) generalizes to protocols augmented with an oracle in the natural way.

A private reduction from an arithmetic two-party functionality $f$ to an arithmetic two-party functionality $g$ is a two party protocol that given an oracle access to the functionality $g$, privately realizes the functionality $f$. Appropriate composition theorems, (e.g. Gol04, Thms. 7.3.3, 7.4.3]), guarantee that the call to $g$ can be replaced by any private protocol realizing $g$, without violating the privacy of the high-level protocol for $f$. 


\subsubsection{The Malicious Model}

We define arithmetic secure two party computation in the malicious setting using the Real-Ideal paradigm. Following our general convention, an honest party in this model is an arithmetic circuit, while a malicious party is allowed to be any polynomial size binary circuit. Formally, we say that an arithmetic protocol $(A, B)$ securely realizes an arithmetic (possibly randomized) two-party functionality $f$ in the malicious setting (in short, $(A, B)$ securely realizes $f$ ) if for any efficient field family $\mathbb{F}=\left\{\mathbb{F}_{n}\right\}$ the binary protocol $(A, B)^{\mathbb{F}}$ securely realizes the binary two-party functionality $f^{\mathbb{F}}$. For completeness, we briefly review the standard definitions of maliciously secure two-computation in the binary model. (See [Gol04, Chapter 7] for more detailed and concrete definitions.)

Let $f(x, y)$ be a two-party functionality, i.e., a (possibly randomized) mapping from a pair of inputs of equal length into a pair of outputs. Let $\pi$ be an two-party protocol. We formulate the requirement that $\pi$ securely computes $f$ by comparing the following "real process" and "ideal process".

The real process. An adversary $A^{*}$ attacking Alice in the real process is a family of probabilistic polynomial-size circuits, who observe all the internal data of Alice, and in addition, has full control over the messages sent by Alice. At the end of the interaction, the adversary may output an arbitrary function of its view, which consists of the inputs, the random coin tosses, and the incoming messages of the corrupted parties. Given a pair of inputs $(x, y) \in\left(\{0,1\}^{n}\right)^{2}$ and an auxiliary input for the adversary aux $\in\{0,1\}^{*}$, the output of the real process is defined as the random variable containing the concatenation of the adversary's output with the outputs and identities of the uncorrupted party. We denote this output by $\operatorname{Real}_{\pi, A^{*}(\operatorname{aux})}(x, y)$. The case of an adversary $B^{*}$

that attacks Bob is defined similarly and in this case we let $\operatorname{Real}_{\pi, B^{*}(\operatorname{aux})}(x, y)$ denote the output of the real process.

The ideal process. In the ideal process, an incorruptible trusted party is employed for computing the given functionality. That is, the "protocol" in the ideal process instructs each party to send its input to the trusted party, who computes the functionality $f$ and sends to each party its output. The interaction of an adversary $\operatorname{Sim}_{A}$, which attacks Alice, (resp., Sim $B$ which attacks $B$ ) with the ideal process and the output of the ideal process are defined analogously to the above definitions for the real process. Except that we allow the adversary to send a special "abort" message (possibly after receiving his output), and, in this case the trusted party sends an abort message to the honest party who halts with a special abort symbol. The adversary attacking the ideal process will also be referred to as a simulator. We denote the output of the ideal process on the inputs $(x, y) \in\left(\{0,1\}^{n}\right)^{2}$ and auxiliary input aux by $\operatorname{Ideal}_{f, \operatorname{Sim}_{A}(\operatorname{aux})}(x, y)$ (resp., $\left.\operatorname{Ideal}_{f, \operatorname{Sim}_{B}(\operatorname{aux})}(x, y)\right)$.

Definition 4.9 (Maliciously Secure Two-Party Computation). The protocol $\pi$ is said to securely realize the given functionality $f$ if for any probabilistic polynomial-size circuit $A^{*}$ attacking Alice (resp., $B^{*}$ attacking Bob) in the real process there exists a probabilistic polynomial-size circuit simulator $\operatorname{Sim}_{A}$ attacking Alice (resp., $\operatorname{Sim}_{B}$ attacking Bob) in the ideal process, such that

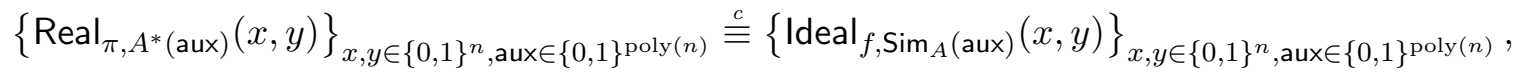

and similarly for Bob:

$$
\left\{\operatorname{Real}_{\pi, B^{*}(\operatorname{aux})}(x, y)\right\}_{x, y \in\{0,1\}^{n}, \operatorname{aux} \in\{0,1\}^{\text {poly }(n)}} \stackrel{c}{=}\left\{\operatorname{Ideal}_{f, \operatorname{Sim}_{B}(\operatorname{aux})}(x, y)\right\}_{x, y \in\{0,1\}^{n}, \operatorname{aux} \in\{0,1\}^{\text {poly }(n)}} .
$$


An arithmetic protocol $(A, B)$ securely realizes an arithmetic (possibly randomized) two-party functionality $f$ if for any efficient field family $\mathbb{F}=\left\{\mathbb{F}_{n}\right\}$ the binary protocol $(A, B)^{\mathbb{F}}$ securely realizes the binary two-party functionality $f^{\mathbb{F}}$

Secure reductions (in the malicious model) are defined analogously to private reductions (in the semi-honest model).

\section{Part I}

\section{Lower Bounds}

In the following sections we will show that some cryptographic primitives cannot be realized in the arithmetic setting. Section 5 is dedicated to Garbed Circuits (formalized under the framework of Randomized Encodings), Section 6 discusses communication lower-bounds for Secure Computation protocols, and Section 7 is devoted to Homomorphic Encryption.

\section{$5 \quad$ Garbled Circuits and Randomized Encoding}

Recall that a deterministic function $f(x, y)$ is encoded by a two-decomposable randomized arithmetic function $\hat{f}(x, y ; r)=(\hat{x}(x ; r), \hat{y}(y ; r), \hat{r}(r))$ if for every input $(x, y)$ the distribution $\hat{f}(x, y ; r)$, induced by a random choice of the internal randomness $r$ of $\hat{f}$, reveals the value of $f(x, y)$ and nothing else. (See Section 4.4 for relevant definitions.)

We show that an arithmetic 2-decomposable encoding must have large communication. In particular, we prove (in Section 5.1 that the encoding $|\hat{x}|$ of $x$ needs to be as long as the output $|f(x, y)|$.

Theorem 5.1. There exists an arithmetic function $f_{n}(x, y)$ which maps $x \in \mathbb{F}$ and $y \in \mathbb{F}^{2 n}$ to an output in $\mathbb{F}^{n}$ for which in any 2-decomposable arithmetic randomized encoding $\hat{f}_{n}(x, y ; r)=$ $(\hat{x}(x ; r), \hat{y}(y ; r), \hat{r}(r))$ of $f$ the function $\hat{x}$ consists of at least $n$ field elements.

In contrast, in the binary setting, Yao's garbled circuit construction Yao86 provides, for any efficiently computable function $f(x, y)$, a 2-decomposable encoding $\hat{f}_{n}(x, y ; r)=(\hat{x}(x ; r), \hat{y}(y ; r), \hat{r}(r))$ with $|\hat{x}|=|x| \cdot \kappa$ where $\kappa$ is the security parameter which can be taken to $n^{\varepsilon}$ for arbitrary small constant $\varepsilon>0$.

For fully decomposable encoding, we prove (in Section 5.2 that the total online complexity $|\hat{x}|$ needs to be roughly as long as the product of the input length and the output length.

Theorem 5.2. Assuming the existence of a binary PRG, there exists an arithmetic function $f$ : $\mathbb{F}^{3 n} \rightarrow \mathbb{F}^{n}$ for which any fully decomposable arithmetic randomized encoding

$$
\hat{f}(x ; r)=\left(\hat{x}_{1}\left(x_{1} ; r\right), \ldots, \hat{x}_{3 n}\left(x_{3 n} ; r\right), \hat{r}(r)\right)
$$

has online complexity $\sum_{i}\left|\hat{x}_{i}\right|$ of at least $n^{2}$.

Again, in the binary setting, Yao's garbled circuit construction provides, for any efficiently computable function $f(x)$, a fully-decomposable encoding $\hat{f}(x ; r)=\left(\hat{x}_{1}\left(x_{1} ; r\right), \ldots, \hat{x}_{n}\left(x_{3 n} ; r\right), \hat{r}(r)\right)$ with online complexity $\sum_{i}\left|\hat{x}_{i}\right|=\left|x_{i}\right| \cdot n^{\varepsilon}$ for arbitrary small constant $\varepsilon>0$. 


\subsection{Proof of Theorem 5.1}

Overview. We follow the outline sketched in the introduction. Roughly speaking, we will exploit the correctness property $f(x)=\operatorname{Dec}(\hat{f}(x ; r))$ to argue that, over sufficiently large field $\mathbb{F}$, for every fixing of the randomness $\boldsymbol{r}$ the circuits $f$ and Dec $\circ \hat{f}(\cdot ; \boldsymbol{r})$ compute the same rational functions. Therefore, by the chain rule, we have that for any input variable $x_{i}$

$$
\partial_{x_{i}} f(x)=\mathcal{J} \operatorname{Dec}(\hat{f}(x ; \boldsymbol{r})) \cdot \partial_{x_{i}} \hat{f}(x ; \boldsymbol{r}) .
$$

Hence, given Dec and an encoding $\hat{f}(\boldsymbol{x} ; \boldsymbol{r})$ of some point $\boldsymbol{x}$, we can deduce that $\partial_{x_{i}} f(\boldsymbol{x})$ is spanned by the columns of $\mathcal{J} \operatorname{Dec}(\hat{f}(\boldsymbol{x} ; \boldsymbol{r}))$. We will show that if the communication is small, this information violates privacy (for a properly chosen function $f$ ). This argument assumes that $\hat{f}$ and Dec compute rational functions and so their derivatives are well defined. This is not the case when the encoder $\hat{f}$ or decoder Dec use zerocheck gates. To cope with this, we apply the robust zerocheck-removal algorithm $\mathcal{T}$ from Definition 3.12 .

We proceed with a formal proof of Theorem 5.1 .

Proof of Theorem 5.1. Consider the function $f: \mathbb{F} \times\left(\mathbb{F}^{n} \times \mathbb{F}^{n}\right) \rightarrow \mathbb{F}^{n}$ that maps $(x,(y, z))$ to $x y+z$ where $x \in \mathbb{F}$ is a scalar and $y$ and $z$ are vectors of length $n$. Assume towards contradiction that $\hat{f}(x,(y, z) ; r)=(\hat{x}, \hat{y}, \hat{r})$ and Dec form a 2-decomposable arithmetic RE with $|\hat{x}|=m<n$. (Note that $\hat{y}$ depends on $(y, z)$ and $r$.) We will show how to break the security of the RE via the following adversary. (From now on, we will often omit the underlying field $\mathbb{F}$ and the security parameter $n$, when clear from the context, i.e. $f(x):=f_{n}^{\mathbb{F}}(x)$.)

Adversary $\mathcal{A}\left(1^{n}\right)$ :

- Choose a prime $p>2^{2 s+1}$ for $s=\max \operatorname{size}\left\{f_{n}, \operatorname{Dec}_{n} \circ \hat{f}_{n}\right\}$.

- Let $\mathbb{F}=\operatorname{GF}(p)$ and send some standard implementation of $\mathbb{F}$ to the challenger.

- Sample $\boldsymbol{x}_{0} \stackrel{R}{\leftarrow} \mathbb{F}$, together with $\boldsymbol{y}_{0}, \boldsymbol{z}_{0} \stackrel{R}{\leftarrow} \mathbb{F}^{n}$.

- Sample $\boldsymbol{x}_{1} \stackrel{R}{\leftarrow} \mathbb{F}$ together with $\boldsymbol{y}_{1} \stackrel{R}{\leftarrow} \mathbb{F}^{n}$ and compute $\boldsymbol{z}_{1}=\boldsymbol{x}_{0} \boldsymbol{y}_{0}+\boldsymbol{z}_{0}-\boldsymbol{x}_{1} \boldsymbol{y}_{1}$.

- Send $\left(\boldsymbol{x}_{0}, \boldsymbol{y}_{0}, \boldsymbol{z}_{0}\right)$ and $\left(\boldsymbol{x}_{1}, \boldsymbol{y}_{1}, \boldsymbol{z}_{1}\right)$ to the challenger.

- Given an encoding $\boldsymbol{c}=(\hat{\boldsymbol{x}}, \hat{\boldsymbol{y}}, \hat{\boldsymbol{r}})$, define the circuit $B(\hat{x})=\operatorname{Dec}_{n}(\hat{x}, \hat{\boldsymbol{y}}, \hat{\boldsymbol{r}})$.

- Compute the zerocheck-free arithmetic circuit $B^{\prime}=\mathcal{T}(B, \hat{\boldsymbol{x}})$.

- Output 1 if $\boldsymbol{y}_{1}$ is spanned by the columns of $\mathcal{J} B^{\prime}(\hat{\boldsymbol{x}})$; Otherwise output 0 .

Observe that this is a valid adversary as $f\left(\boldsymbol{x}_{0}, \boldsymbol{y}_{0}, \boldsymbol{z}_{0}\right)=f\left(\boldsymbol{x}_{1}, \boldsymbol{y}_{1}, \boldsymbol{z}_{1}\right)$. The following claims show that $\mathcal{A}$ distinguishes between the two cases with advantage of at least $\frac{3}{4}-\operatorname{neg}(n)$.

Claim 5.3. If the challenge bit $b=1$, i.e., the challenger sends a sample $\boldsymbol{c}=\hat{f}\left(\boldsymbol{x}_{1}, \boldsymbol{y}_{1}, \boldsymbol{z}_{1} ; \boldsymbol{r}\right)$ for randomly chosen $\boldsymbol{r}$, then the adversary accepts with probability $1-\operatorname{neg}(n)$.

Proof. Fix the randomness of the challenger to some value $\boldsymbol{r}$ and fix the values $\boldsymbol{y}_{1}, \boldsymbol{z}_{1}$. Let $f_{\boldsymbol{y}_{1}, \boldsymbol{z}_{1}}(x)=f_{n}\left(x, \boldsymbol{y}_{1}, \boldsymbol{z}_{1}\right)$, let $A(x)$ denote the circuit $\hat{f}_{n}\left(x, \boldsymbol{y}_{1}, \boldsymbol{z}_{1} ; \boldsymbol{r}\right)$, and let $B(\hat{x})=\operatorname{Dec}_{n}\left(\hat{x}, \hat{\boldsymbol{y}}_{1}, \hat{\boldsymbol{r}}_{1}\right)$, where $\hat{\boldsymbol{y}}_{1}$ and $\hat{\boldsymbol{r}}$ are the outcome of $\hat{f}$ when applied to $\boldsymbol{y}_{1}, \boldsymbol{z}_{1}$ and $\boldsymbol{r}$. (Due to the decomposability these values are independent of $\hat{x}$.) Syntactically,

$$
f_{\boldsymbol{y}_{1}, \boldsymbol{z}_{1}}: \mathbb{F} \rightarrow \mathbb{F}^{n}, \quad A: \mathbb{F}^{n} \rightarrow \mathbb{F}^{m}, \quad B: \mathbb{F}^{m} \rightarrow \mathbb{F}^{n} .
$$


By the perfect correctness and the decomposability property, it holds that $B(A(x))=f_{\boldsymbol{y}_{1}, \boldsymbol{z}_{1}}(x)$ for every $x$. Hence, by Lemma 3.13 , we have

$$
\operatorname{Pr}_{\substack{R \\ \boldsymbol{x}_{1}}}\left[\partial_{x} f_{\boldsymbol{y}_{1}, \boldsymbol{z}_{1}}\left(\boldsymbol{x}_{1}\right)=\mathcal{J} B^{\prime}\left(A\left(\boldsymbol{x}_{1}\right)\right) \cdot \partial_{x} A^{\prime}\left(\boldsymbol{x}_{1}\right)\right] \geq 1-\operatorname{neg}(n),
$$

where $A^{\prime}=\mathcal{T}\left(A, \boldsymbol{x}_{1}\right)$ and $B^{\prime}=\mathcal{T}\left(B, \hat{\boldsymbol{x}}_{1}\right)$. Since $\boldsymbol{y}_{1}=\partial_{x} f_{\boldsymbol{y}_{1}, \boldsymbol{z}_{1}}\left(\boldsymbol{x}_{1}\right)$ and $\boldsymbol{c}=A\left(\boldsymbol{x}_{1}\right)$, we conclude that

$$
\operatorname{Pr}_{\boldsymbol{x}_{1} \leftarrow \mathbb{R}}\left[\boldsymbol{y}_{1} \in \operatorname{colSpan}\left(\mathcal{J} B^{\prime}(\boldsymbol{c})\right)\right] \geq 1-\operatorname{neg}(n)
$$

and the claim follows.

We move on to the case where $b=0$.

Claim 5.4. If the challenge bit $b=0$, i.e., the challenger sends a sample $\boldsymbol{c}=\hat{f}\left(\boldsymbol{x}_{0}, \boldsymbol{y}_{0}, \boldsymbol{z}_{0} ; \boldsymbol{r}\right)$ for randomly chosen $\boldsymbol{r}$, then the adversary accepts with probability at most $1 / 4$.

Proof. Fix $\boldsymbol{x}_{0}, \boldsymbol{y}_{0}, \boldsymbol{z}_{0}$ as well as the randomness of the challenger and note that $\boldsymbol{y}_{1}$ is still uniformly distributed. Let $M$ denote the matrix $\mathcal{J} B^{\prime}\left(\hat{f}_{r}\left(\boldsymbol{x}_{0}, \boldsymbol{y}_{0}, \boldsymbol{z}_{0}\right)\right)$. Since the input $\hat{x}$ of $B^{\prime}$ is of length $m<n$, the matrix $M$ is of dimensions $n \times m$, and its columns span at most $|\mathbb{F}|^{m}$ vectors in $\mathbb{F}^{n}$. It follows that

$$
\underset{\boldsymbol{y}_{1} \stackrel{R}{\leftarrow} \mathbb{F}^{n}}{\operatorname{Pr}}\left[\boldsymbol{y}_{1} \text { is spanned by the columns of } M\right] \leq|\mathbb{F}|^{m-n},
$$

and so $\mathcal{A}$ outputs 1 with probability at most $|\mathbb{F}|^{m-n}$ which is upper-bounded by $1 / 4$ for $m<n$.

Overall, $\mathcal{A}$ wins the game with probability greater than $1-\operatorname{neg}(n)-1 / 4>3 / 4-\operatorname{neg}(n)$, and the theorem follows.

\subsection{Proof of Theorem 5.2}

Let $\mathrm{PRG}=\left\{\mathrm{PRG}_{n}\right\}$ with $\mathrm{PRG}_{n}:\{0,1\}^{n} \rightarrow\{0,1\}^{n^{2}}$ be a pseudorandom generator over the binary field. We can transform the boolean circuit that computes it into an arithmetic circuit by substituting $\operatorname{AND}\left(w_{1}, w_{2}\right)$ gates with $w_{1} \times w_{2}$, and $\operatorname{NOT}(w)$ gates by $1-w$. By abuse of notation, we let $\mathrm{PRG}_{n}: \mathbb{F}^{n} \rightarrow \mathbb{F}^{n^{2}}$ also denote the transformed circuit. Observe that when the input to the circuit consists of $0-1$ values, the functionality is preserved. Specifically, for a random $\boldsymbol{x} \in\{0,1\}^{n}$, the vector PRG $(\boldsymbol{x})$ consists of a pseudorandom sequence of $0-1$ elements.

To prove Theorem 5.2, we will consider the function $f: \mathbb{F}^{n} \times \mathbb{F}^{n} \times \mathbb{F}^{n} \rightarrow \mathbb{F}^{n}$ that maps $(x, y, z)$ to $Y x+z$, where $Y$ is the $n \times n$ matrix which consists of the elements generated by PRG $(y)$. Assume, towards a contradiction, that $f$ is encoded by a fully decomposable arithmetic randomized encoding

$$
\hat{f}(x, y, z ; r)=(\hat{x}, \hat{y}, \hat{z}, \hat{r})
$$

with decoder Dec where $|\hat{x}|$ is shorter than $n^{2}$. Since $\hat{f}$ is fully decomposable $\hat{x}=\left(\hat{x}_{1}, \ldots, \hat{x}_{n}\right)$, and, by an averaging argument, at least some $\hat{x}_{i}$ is shorter than $n$. We will exploit this to property to break the original binary PRG. Specifically, we define the following adversary $\mathcal{A}$.

Adversary $\mathcal{A}\left(1^{n}, Y \in\{0,1\}^{n^{2}}\right)$ :

- Choose a prime $p>2^{2 s+1}$ for $s=\max \operatorname{size}\left\{f_{n}, \operatorname{Dec}_{n} \circ \hat{f}_{n}\right\}$ and let $\mathbb{F}=\operatorname{GF}(p)$. 
- Parse the challenge $Y \in\{0,1\}^{n^{2}}$ into columns $\left(Y_{1}, \ldots, Y_{n}\right)$.

- Sample a point $\boldsymbol{x}, \boldsymbol{z} \stackrel{R}{\leftarrow} \mathbb{F}^{n}$ at random and fix $\boldsymbol{y}$ to some default value, e.g., $0^{n}$. Sample randomness $\boldsymbol{r}$ for the encoding $\hat{f}_{n}$ and let $(\hat{\boldsymbol{x}}, \hat{\boldsymbol{y}}, \hat{\boldsymbol{z}}, \hat{\boldsymbol{r}})=\hat{f}(\boldsymbol{x}, \boldsymbol{y}, \boldsymbol{z} ; \boldsymbol{r})$.

- Compute $\operatorname{Dec}^{\prime}:=\mathcal{T}(\operatorname{Dec},(\hat{\boldsymbol{x}}, \hat{\boldsymbol{y}}, \hat{\boldsymbol{z}}, \hat{\boldsymbol{r}}))$.

- Output 1 if $Y_{i} \in \operatorname{colSpan}\left(\mathcal{J}_{\hat{x}_{i}} \operatorname{Dec}^{\prime}((\hat{\boldsymbol{x}}, \hat{\boldsymbol{y}}, \hat{\boldsymbol{z}}, \hat{\boldsymbol{r}}))\right)$, for all $i=1, \ldots, n$; Otherwise, output 0 .

Analysis of the Adversary. We will show that the adversary $\mathcal{A}$ accepts, with probability $1-\operatorname{neg}(n)$, a pseudorandom string $Y=\operatorname{PRG}(\boldsymbol{y})$ where $\boldsymbol{y} \stackrel{R}{\leftarrow}\{0,1\}^{n}$. We will prove this in two steps. First, we will show that this is the case if the adversary sets $\boldsymbol{y}$ to be the seed of $Y$ (Claim 5.5), and then we show (by relying on the security of the encoding) that this is the case even when $\boldsymbol{y}$ is set to an arbitrary value (Claim 5.6). We will later show (Claim 5.7) that a random $Y \in\{0,1\}^{n^{2}}$ is accepted with probability $\frac{1}{2}$, and conclude that $\mathcal{A}$ breaks the PRG.

Claim 5.5. For any fixed $\boldsymbol{r}, \boldsymbol{y}, \boldsymbol{z}$, it holds, with overwhelming probability over $\boldsymbol{x}$, that for every $i$ :

$$
Y_{i} \in \operatorname{colSpan}\left(\mathcal{J}_{\hat{x}_{i}} \operatorname{Dec}^{\prime}(\hat{f}(\boldsymbol{x}, \boldsymbol{y}, \boldsymbol{z} ; \boldsymbol{r}))\right),
$$

where $Y=\operatorname{PRG}(\boldsymbol{y}), \operatorname{Dec}^{\prime}:=\mathcal{T}(\operatorname{Dec}, \hat{f}(\boldsymbol{x}, \boldsymbol{y}, \boldsymbol{z} ; \boldsymbol{r}))$.

Proof. Let $A(x)=\hat{f}_{\boldsymbol{y}, \boldsymbol{z}, \boldsymbol{r}}(x)$. By perfect correctness, $f_{\boldsymbol{y}, \boldsymbol{z}}(x)=\operatorname{Dec}(A(x))$ for every $x$. Hence, by Lemma 3.13 , with overwhelming probability over $\boldsymbol{x}$, for every $i$ we have

$$
\partial_{x_{i}} f_{\boldsymbol{y}, \boldsymbol{z}}(\boldsymbol{x})=\mathcal{J} \operatorname{Dec}^{\prime}(A(\boldsymbol{x})) \cdot \partial_{x_{i}} A^{\prime}(\boldsymbol{x}),
$$

where $A^{\prime}$ is the circuit outputted by $\mathcal{T}(A, \boldsymbol{x})$ and $\operatorname{Dec}^{\prime}$ is the circuit outputted by $\mathcal{T}(\mathrm{Dec}, \boldsymbol{x})$. (The circuit $A^{\prime}$ is unknown to the attacker, who does not know $\boldsymbol{r}$, yet such a circuit exists.)

Fix some $i \in\{1, \ldots, n\}$. Observe that $A\left(x_{1}, \ldots, x_{n}\right)=\left(\hat{x}_{1}, \ldots, \hat{x}_{n}, \hat{\boldsymbol{y}}, \hat{\boldsymbol{z}}, \hat{\boldsymbol{r}}\right)$ and the only output that depends on $x_{i}$ is $\hat{x}_{i}$ (since the encoding is decomposable). Hence, when deriving $A^{\prime}$ with respect to $x_{i}$, all the entries that do not correspond to the outputs $\hat{x}_{i}$ vanish. The above equation therefore simplifies to

$$
\partial_{x_{i}} f_{\boldsymbol{y}, \boldsymbol{z}}(\boldsymbol{x})=\mathcal{J}_{\hat{x}_{i}} \operatorname{Dec}^{\prime}(A(\boldsymbol{x})) \cdot \partial_{x_{i}} A^{\prime}
$$

Recalling that $Y_{i}=\partial_{x_{i}} f_{\boldsymbol{y}, \boldsymbol{z}}(\boldsymbol{x})$, completes the proof.

We move on to analyze the probability that $\mathcal{A}$ accepts a pseudorandom $Y$.

Claim 5.6. It holds that

$$
\operatorname{Pr}_{\boldsymbol{y}_{1} \stackrel{R}{\leftarrow}\{0,1\}^{n}}\left[\mathcal{A}\left(Y^{1}\right)=1\right]=1-\operatorname{neg}(n),
$$

where $Y^{1}=\operatorname{PRG}_{n}\left(\boldsymbol{y}_{1}\right)$.

Proof. Assume, towards a contradiction, that the claim does not hold and, for $\boldsymbol{y}_{1} \stackrel{R}{\leftarrow}\{0,1\}^{n}$, the adversary $\mathcal{A}$ accepts $Y^{1}=\operatorname{PRG}\left(\boldsymbol{y}_{1}\right)$ with probability $1-\varepsilon(n)$ for some non-negligible $\varepsilon$. Consider the following adversary $\mathcal{B}\left(1^{n}\right)$ that attacks the computational privacy of randomized encodings:

Adversary $\mathcal{B}\left(1^{n}\right)$ : 
- Choose a prime $p>2^{2 s+1}$ for $s=\max \operatorname{size}\left\{f_{n}, \operatorname{Dec}_{n} \circ \hat{f}_{n}\right\}$.

- Let $\mathbb{F}=\operatorname{GF}(p)$ and send some standard implementation of $\mathbb{F}$ to the challenger.

- Sample $\boldsymbol{x}_{0}, \boldsymbol{z}_{0} \stackrel{R}{\leftarrow} \mathbb{F}^{n}$, let $\boldsymbol{y}_{0}=0^{n}$ and $Y^{0}=\operatorname{PRG}\left(\boldsymbol{y}_{0}\right)$.

- Sample $\boldsymbol{x}_{1} \stackrel{R}{\leftarrow} \mathbb{F}^{n}, \boldsymbol{y}_{1} \stackrel{R}{\leftarrow}\{0,1\}^{n}$, let $Y^{1}=\operatorname{PRG}\left(\boldsymbol{y}_{1}\right)$ and $\boldsymbol{z}_{1}=Y^{0} \boldsymbol{x}_{0}+\boldsymbol{z}_{0}-Y^{1} \boldsymbol{x}_{1}$.

- Send $\left(\boldsymbol{x}_{0}, \boldsymbol{y}_{0}, \boldsymbol{z}_{0}\right)$ and $\left(\boldsymbol{x}_{1}, \boldsymbol{y}_{1}, \boldsymbol{z}_{1}\right)$ to the challenger.

- On input challenge $\boldsymbol{c}$, let $\operatorname{Dec}^{\prime}:=\mathcal{T}(\operatorname{Dec}, \boldsymbol{c})$.

- Output 1 if $Y_{i}^{1} \in \operatorname{colSpan}\left(\mathcal{J}_{\hat{x}_{i}} \operatorname{Dec}^{\prime}(\boldsymbol{c})\right)$ for all $i=1, \ldots, n$. Otherwise, output 0 .

Observe that $f$ agrees on the 0 -inputs $\left(\boldsymbol{x}_{0}, \boldsymbol{y}_{0}, \boldsymbol{z}_{0}\right)$ and on the 1-inputs $\left(\boldsymbol{x}_{1}, \boldsymbol{y}_{1}, \boldsymbol{z}_{1}\right)$, and so $\mathcal{B}$ is a valid adversary. When $b=1$, by Claim 5.5, $\mathcal{B}$ outputs 1 with overwhelming probability. When $b=0$, the probability that $\mathcal{B}$ returns 1 is exactly $1-\varepsilon(n)$, since this is the probability that $\mathcal{A}$ accepts $Y_{1}=\operatorname{PRG}\left(\boldsymbol{y}_{1}\right)$ for $\boldsymbol{y}_{1} \stackrel{R}{\leftarrow}\{0,1\}^{n}$. (Indeed, observe that in both $\mathcal{A}$ and $\mathcal{B}$ the values $\boldsymbol{x}_{1}, \boldsymbol{z}_{1}$ are uniform over $\mathbb{F}^{n}$.) Overall the advantage of $\mathcal{B}$ is $\varepsilon(n)$, which, under our assumption, is non-negligible, contradicting the computational privacy of the randomized encoding.

We move on to show that if the encoding $\hat{x}$ is shorter than $n^{2}$, a random binary string $Y \in$ $\{0,1\}^{n^{2}}$ is unlikely to be accepted by $\mathcal{A}$. As in the previous section, the proof uses a dimension argument.

Claim 5.7. It holds that $\operatorname{Pr}_{Y \stackrel{R}{\leftarrow}\{0,1\}^{n}}[\mathcal{A}(Y)=1] \leq \frac{1}{2}$.

Proof. Since $\hat{x}$ is shorter than $n^{2}$, there must be some $i \in\{1, \ldots, n\}$ for which $\hat{x}_{i}$ is shorter than $n$. We will focus on this $i$ and show that for every fixed randomness $\boldsymbol{r}$ and for every $\boldsymbol{x}, \boldsymbol{y}, \boldsymbol{z} \in \mathbb{F}^{n}$ it holds that:

$$
\underset{Y \stackrel{R}{\leftarrow}\{0,1\}^{n^{2}}}{\operatorname{Pr}}\left[Y_{i} \in \operatorname{colSpan}\left(\mathcal{J}_{\hat{x}_{i}} \operatorname{Dec}^{\prime}(\hat{f}(\boldsymbol{x}, \boldsymbol{y}, \boldsymbol{z} ; \boldsymbol{r}))\right)\right] \leq \frac{1}{2} .
$$

Indeed, Dec has $n$ outputs, and so $M:=\mathcal{J}_{\hat{x}_{i}} \operatorname{Dec}^{\prime}(\hat{f}(\boldsymbol{x}, \boldsymbol{y}, \boldsymbol{z} ; \boldsymbol{r}))$ is a $n \times\left|\hat{x}_{i}\right|$ matrix of elements in $\mathbb{F}$. Since $\left|\hat{x}_{i}\right|<n$, there exists a non-trivial vector $\boldsymbol{u} \in \mathbb{F}^{n}$ in the Kernel of $M$, i.e., $M \cdot \boldsymbol{u}=0^{\left|\hat{x}_{i}\right|}$. Let $j$ denote some non-zero coordinate of $\boldsymbol{u}$, namely, $\boldsymbol{u}_{j} \neq 0$. For every vector $\boldsymbol{v} \in \operatorname{colSpan}(M)$, it holds that $\boldsymbol{u}^{\mathrm{T}} \cdot \boldsymbol{v}=0$. We will establish Eq. (8) by showing that

$$
\underset{Y_{i} \stackrel{R}{\leftarrow}\{0,1\}^{n}}{\operatorname{Pr}}\left[\boldsymbol{u}^{\mathrm{T}} \cdot Y_{i}=0\right] \leq \frac{1}{2} .
$$

To see this observe that for any $w \in\{0,1\}^{n}$ which is orthogonal to $\boldsymbol{u}$, one can find a unique vector $w^{\prime}$ which is not orthogonal to $\boldsymbol{u}$ by letting $w^{\prime}=w \oplus e_{j}$. (Here $e_{j}$ denotes the $j$-th unit vector, whose $j$-th coordinate equals to 1 , and whose other coordinates are equal to 0 .) Indeed, if $\boldsymbol{u}^{\mathrm{T}} \cdot w=0$ then $\boldsymbol{u}^{T} \cdot w^{\prime} \in\left\{ \pm \boldsymbol{u}_{j}\right\}$. The claim follows.

Overall, $\mathcal{A}$ accepts random bitstrings with probability less than $1 / 2$, and pseudorandom strings with probability $1-\operatorname{neg}(n)$, and so it breaks the security of the PRG. 


\section{Arithmetic Multiparty Computation}

In this section will prove lower-bounds on the online communication of secure two-party computation in the arithmetic model.

\subsection{Definitions}

Secure computation with one-sided privacy. We consider a minimal form of Secure Computation with one-sided privacy. (This restriction only makes our lower bounds stronger.) Specifically, we will consider two-party functionalities in which only one party receives the output. Namely, Alice holds input $x$, Bob holds input $y$, and we want Alice to learn the functionality $f(x, y)$ without learning anything else. We require only one-sided security, and so Bob may learn everything. This notion of security is formalized below.

Recall that a two-party arithmetic protocol $\Pi$ is a pair of (uniform) polynomial size arithmetic circuits $A=\left\{A_{n}\right\}$ and $B=\left\{B_{n}\right\}$, which communicate with each other via special Send and Receive gates. Further recall that, for a field $\mathbb{F}$ and inputs $x, y \in \mathbb{F}^{n}$, the output of $A$ is denoted by

Output $^{\Pi, \mathbb{F}_{n}}(x, y)$ and the view of $A$ is denoted by $\operatorname{View}_{A}^{\Pi, \mathbb{F}}(x, y)$. (The latter is the random variable which consists of the deterministic and random inputs of $A_{n}$ and all the messages sent by $B_{n}$.)

Definition 6.1 (Arithmetic Two-Party Computation with One-Sided Semi-Honest Security). We say that a two party arithmetic protocol $\Pi=\left(\left\{\right.\right.$ Alice $\left._{n}\right\},\left\{\right.$ Bob $\left.\left._{n}\right\}\right)$ realizes a (deterministic) functionality $f: \mathbb{F}^{*} \times \mathbb{F}^{*} \rightarrow \mathbb{F}^{*}$ with one-sided privacy if the following hold:

- Correctness: For every field $\mathbb{F}$ and inputs $x, y \in \mathbb{F}^{n}$,

$$
\operatorname{Pr}\left[\text { Output }{ }^{\Pi, \mathbb{F}}(x, y) \neq f^{\mathbb{F}}(x, y)\right]=0 .
$$

- One-sided Privacy (against a semi-honest Alice): For each polynomial-time Turing machine adversary $\mathcal{A}$, there exist a probabilistic polynomial-time Turing machine Sim such that the adversary $\mathcal{A}\left(1^{n}\right)$ can win the following game with probability at most $1 / 2+n e g(n)$ :

- Given $1^{n}$, the adversary $\mathcal{A}$ picks a field implementation $\mathbb{F}$, inputs $\boldsymbol{x}, \boldsymbol{y} \in \mathbb{F}^{\text {poly }(n)}$, and outputs $\mathbb{F}, \boldsymbol{x}, \boldsymbol{y}$.

- The challenger samples $b \stackrel{R}{\leftarrow}\{0,1\}$ and returns a view $z$ where $z \stackrel{R}{\leftarrow} \operatorname{View}_{A}^{\Pi, \mathbb{F}}(\boldsymbol{x}, \boldsymbol{y})$ if $b=0$; and $z \stackrel{R}{\leftarrow} \operatorname{Sim}(\boldsymbol{x}, f(\boldsymbol{x}, \boldsymbol{y}), \mathbb{F})$ if $b=1$.

- The adversary $\mathcal{A}$ outputs a bit $b^{\prime}$ and wins if $b^{\prime}=b$.

Note that the above definition is uniform in the sense that the inputs $x, y$ are chosen by a uniform adversary. Again, this only makes our lower-bound stronger.

Clearly, there is a trivial protocol that achieves one-sided privacy: Alice sends her input $x$ to Bob, who computes the function $f(x, y)$, and returns the result to Alice. In this protocol Alice receives $m$ field elements, where $m$ is the output length of $f$. We will show that this is necessary even if the parties are allowed to exchange an arbitrary number of messages at an offline phase.

Online/Offline MPC. Without loss of generality, we assume that a protocol $\Pi=$ (Alice, Bob) has the following format. The parties Alice and Bob use the randomness $r_{a}, r_{b}$ respectively. They first run the offline phase of the MPC protocol. We denote by $\hat{r}\left(r_{a}, r_{b}\right)$ the transcript of all the 
messages that Alice gets during the offline phase. After the offline phase, Alice receives an input $x$ and Bob receives an input $y$. They exchange some further messages. We denote the messages sent by Bob as $\hat{t}=\hat{t}\left(r_{a}, r_{b}, x, y\right)$ and let $|\hat{t}|$ denote the incoming online communication complexity of Alice. (For simplicity, we assume that this amount depends only on the security parameter and not on the messages themselves.) At the end of the protocol, Alice runs a final algorithm on her view $\left(x, r_{a}, \hat{r}\left(r_{a}, r_{b}\right), \hat{t}\left(r_{a}, r_{b}, x, y\right)\right)$ to get the output of the protocol. We refer to this final algorithm as the decoder, $\operatorname{Dec}\left(x, r_{a}, \hat{r}, \hat{t}\right)$.

\subsection{Main Result}

We will prove that the online communication from Bob to Alice is as long as the output length of $f$. This lower-bound applies even to the case where $f$ is strictly arithmetic, i.e., it does not contain division, zerocheck gates or bitsample gates and simply computes a polynomial over $\mathbb{F}$.

Our lower-bound relies on the existence of an arithmetic pseudorandom generator (APRG) (see Definition 4.1). Recall that the output of an APRG is pseudorandom over $\mathbb{F}^{\ell}$ and its input (the seed) may contain both random field elements and random bits as long their total number is $n<\ell$. We will later (Section 9) show that APRG with polynomial stretch can be constructed based on the RLC assumption (with constant rate and constant noise rate). Furthermore, this APRG is simple, i.e., it does not contain division or zerocheck gates (but uses random bits).

Theorem 6.2. Assume the existence of a simple arithmetic PRG. Then, for any arbitrary constant $c>1$, there is a strictly arithmetic function $f: \mathbb{F} \times \mathbb{F}^{n} \rightarrow \mathbb{F}^{n^{c}}$ that cannot be realized with onesided semi-honest security by an arithmetic two-party protocol $\Pi$ for which the incoming online communication complexity of Alice is shorter than $n^{c}-1$ field elements.

Remark 6.3 (The multiparty case). A similar bound on the communication applies in the multiparty setting even in the case of honest majority. Indeed, any t-party protocol $\Pi$ for $f$ in which the first two parties play the roles of Alice and Bob and all the other parties act as "servers" with no input or output, immediately yields a two party protocol $\Pi^{\prime}$ with one-sided semi-honest security even if $\Pi$ is only semi-honest secure for coalitions of size 1.

Proof of Theorem 6.2. Let $m=n^{c}$. We will later show (Lemma 9.2) that the existence of any (simple) APRG implies the existence of a (simple) APRG with seed of length $n$ and output length of $m$. We view APRG as an arithmetic function APRG $: \mathbb{F}^{n} \rightarrow \mathbb{F}^{m}$ by thinking of the random gates as inputs. This is done even for gates that sample random bits. We follow the convention that whenever a random seed $\boldsymbol{y}$ is chosen, it is chosen from the "right" distribution, i.e., binary inputs are chosen as random $0-1$ values. As a result, $Y=\operatorname{APRG}(\boldsymbol{y})$ is pseudorandom over $\mathbb{F}^{2 m}$. (Note however, that the pseudorandomness of the function $A P R G_{n}$ is not guaranteed for a $y$ sampled from other distributions.)

We consider the function $f(x, y)=Y_{0} x+Y_{1}$ where $\left(Y_{0}, Y_{1}\right)=\operatorname{APRG}(y)$. Alice's input to the function is $x$, while Bob holds $y$. Our proof shows that if APRG is a strictly arithmetic PRG, then we can break the computational privacy of any MPC protocol where the online communication $\ell$ from Bob to Alice is (strictly) shorter than $m-1$. We define the following adversary against the computational privacy of the MPC protocol. As in the previous section, we use subscripts to denote fixed inputs (e.g., $f_{\boldsymbol{y}}(x)=f(x, \boldsymbol{y})$ ).

\section{Distinguisher $\mathcal{A}$ :}


- Let $\mathbb{F}=\mathrm{GF}(p)$ for a prime $p>2 \cdot 2^{2 s}$ where $s$ is an upper bound on the accumulated size of all the circuits in Alice, Bob and Dec, and the circuit which computes $f$.

- Sample $\boldsymbol{x} \stackrel{R}{\leftarrow} \mathbb{F}$.

- Sample a random seed $\boldsymbol{y}$ for the APRG and compute $\left(Y_{0}, Y_{1}\right):=\operatorname{APRG}(\boldsymbol{y})$.

- Send to the challenger an implementation of $\mathbb{F}$ and the inputs $(\boldsymbol{x}, \boldsymbol{y})$.

- Given a challenge $\left(\boldsymbol{x}, \boldsymbol{r}_{a}, \hat{\boldsymbol{r}}, \hat{\boldsymbol{t}}\right)$, call the procedure SpanCheck $\left(Y_{0},\left(\boldsymbol{x}, \boldsymbol{r}_{a}, \hat{\boldsymbol{r}}, \hat{\boldsymbol{t}}\right)\right)$ defined as follows:

- Let $B(x, \hat{t})$ denote the computation of the decoder with the values $\boldsymbol{r}_{a}$ and $\hat{\boldsymbol{r}}$ fixed, i.e., $\operatorname{Dec}_{\boldsymbol{r}_{a}, \hat{\boldsymbol{r}}}(x, \hat{t})$.

- Compute $B^{\prime}=\mathcal{T}(B,(\boldsymbol{x}, \hat{\boldsymbol{t}}))$, where $\mathcal{T}$ is the zero-check removal procedure from Lemma 3.13 .

- If $Y_{0} \in \operatorname{colSpan} \mathcal{J} B^{\prime}(\boldsymbol{x}, \hat{\boldsymbol{t}})$ return 0; Otherwise, return 1.

In the following, we will show that if $\left(\boldsymbol{x}, \boldsymbol{r}_{a}, \hat{\boldsymbol{r}}, \hat{\boldsymbol{t}}\right)=\operatorname{View}_{A}\left(\boldsymbol{r}_{a}, \boldsymbol{r}_{b}, \boldsymbol{x}, \boldsymbol{y}\right)$ (i.e., the challenge bit $b$ is zero), then $\mathcal{A}$ returns 0 with overwhelming probability (Claim 6.4). On the other hand, we will show (Claim 6.6) that if $\left(\boldsymbol{x}, \boldsymbol{r}_{a}, \hat{\boldsymbol{r}}, \hat{\boldsymbol{t}}\right) \stackrel{R}{\leftarrow} \operatorname{Sim}\left(\boldsymbol{x}, Y_{0} \boldsymbol{x}+Y_{1}, \mathbb{F}\right)$ (i.e., the challenge bit $b$ is one), $\mathcal{A}$ errs (outputs 0 ) with probability at most $1 / 2+\operatorname{neg}(n)$.

Claim 6.4. For every fixed $\boldsymbol{y} \in \mathbb{F}^{n}$ and fixed randomness $\boldsymbol{r}_{a}$ and $\boldsymbol{r}_{b}$, it holds that

$$
\operatorname{Pr}_{\boldsymbol{x} \in \mathbb{F}}\left[Y_{0} \in \operatorname{colSpan} \mathcal{J} B^{\prime}(\boldsymbol{x}, \hat{\boldsymbol{t}})\right] \geq 1-\operatorname{neg}(n),
$$

where $Y_{0}$ is the first half of the output of $\operatorname{APRG}(\boldsymbol{y})$, and $B^{\prime}$ is defined as in $\mathcal{A}$, i.e., $B^{\prime}=\mathcal{T}\left(\operatorname{Dec}_{\boldsymbol{r}_{a}, \hat{\boldsymbol{r}}},(\boldsymbol{x}, \hat{\boldsymbol{t}})\right)$ where $\left(\boldsymbol{x}, \boldsymbol{r}_{a}, \hat{\boldsymbol{r}}, \hat{\boldsymbol{t}}\right)=\operatorname{View}_{A}\left(\boldsymbol{r}_{a}, \boldsymbol{r}_{b}, \boldsymbol{x}, \boldsymbol{y}\right)$.

Proof. Fix $\boldsymbol{r}_{a}, \boldsymbol{r}_{b}$ and $\boldsymbol{y}$, and consider the arithmetic circuit $A(x)$ that outputs $(x, \hat{t})$ where $\hat{t}$ denotes Bob's online messages that correspond to the inputs $\left(\boldsymbol{r}_{a}, \boldsymbol{r}_{b}, x, \boldsymbol{y}\right)$. The circuit $A$ can be obtained by composing the subcircuits of Alice and Bob, and therefore, $A$ is of size is smaller than $s$. From perfect correctness we know that

$$
f_{\boldsymbol{y}}(x)=B(A(x))
$$

where $f_{\boldsymbol{y}}(x)=f(x, \boldsymbol{y})$. Since the mapping APRG is strictly arithmetic, so is $f_{\boldsymbol{y}}(x)$. Therefore, we can apply Lemma 3.13 , for the field $\mathbb{F}$, and get that

$$
\underset{\boldsymbol{x} \leftarrow \mathbb{R}}{\operatorname{Pr}}\left[\partial_{x} f_{\boldsymbol{y}}(\boldsymbol{x})=\mathcal{J} B^{\prime}(A(\boldsymbol{x})) \cdot \partial_{x} A^{\prime}(\boldsymbol{x})\right] \geq 1-\operatorname{neg}(n)
$$

where $A^{\prime}:=\mathcal{T}(A, \boldsymbol{x})$. (Note that, by definition, $B^{\prime}:=\mathcal{T}(B, A(\boldsymbol{x}))$.)

Plugging in the definitions of $A$ and $f$ yields that, with overwhelming probability over $\boldsymbol{x}$,

$$
Y_{0}=\mathcal{J} B^{\prime}(\boldsymbol{x}, \hat{\boldsymbol{t}}) \cdot \partial_{x} A^{\prime}(\boldsymbol{x}),
$$

and the claim follows.

We showed that if $b=0$, then the adversary returns 0 and wins with overwhelming probability. We now prove that if $b=1$, the adversary returns 0 with probability at most $\frac{1}{2}+\operatorname{neg}(n)$. For this we focus on the SpanCheck subroutine of $\mathcal{A}$. We will analyze the acceptance probability of SpanCheck on three different (hybrid) distributions, where the first distribution corresponds to the distribution used by $\mathcal{A}$ when $b=1$. The hybrid distributions are defined in Table 1 . 


\begin{tabular}{|l|l|l|l|}
\hline & $\mathcal{D}_{1}$ & $\mathcal{D}_{2}$ & $\mathcal{D}_{3}$ \\
\hline & Sample random seed $\boldsymbol{y}$ & $\left(Y_{0}, Y_{1}\right) \stackrel{R}{\leftarrow} \mathbb{F}^{2 m}$ & $\left(Y_{0}, Z\right) \stackrel{R}{\leftarrow} \mathbb{F}^{2 m}$ \\
& $\left(Y_{0}, Y_{1}\right)=$ APRG $(\boldsymbol{y})$ & \\
& $\boldsymbol{x} \stackrel{R}{\leftarrow} \mathbb{F}$ & $\left(\boldsymbol{x}, \boldsymbol{r}_{a}, \hat{\boldsymbol{r}}, \hat{\boldsymbol{t}}\right) \stackrel{R}{\leftarrow} \operatorname{Sim}(\boldsymbol{x}, Z, \mathbb{F})$ \\
& $\left(\boldsymbol{x}, \boldsymbol{r}_{a}, \hat{\boldsymbol{r}}, \hat{\boldsymbol{t}}\right) \stackrel{R}{\leftarrow} \operatorname{Sim}\left(\boldsymbol{x}, Y_{0} \boldsymbol{x}+Y_{1}, \mathbb{F}\right)$ & & \\
\hline Output: & $\left(Y_{0},\left(\boldsymbol{x}, \boldsymbol{r}_{a}, \hat{\boldsymbol{r}}, \hat{\boldsymbol{t}}\right)\right)$ & & \\
\hline
\end{tabular}

Table 1: Three distributions, all of which output a tuple $\left(Y_{0},\left(\boldsymbol{x}, \boldsymbol{r}_{a}, \hat{\boldsymbol{r}}, \hat{\boldsymbol{t}}\right)\right)$. In order to avoid cluttering, we only write modifications with respect to the previous distribution. For example, $x$ in $\mathcal{D}_{2}$ is sampled as in $\mathcal{D}_{1}$.

Claim 6.5. For $\left(Y_{0}, \boldsymbol{x}, \boldsymbol{r}_{a}, \hat{\boldsymbol{r}}, \hat{\boldsymbol{t}}\right) \stackrel{R}{\leftarrow} \mathcal{D}_{3}$ it holds that:

$$
\operatorname{Pr}\left[Y_{0} \in \operatorname{colSpan} \mathcal{J} B^{\prime}(\boldsymbol{x}, \hat{\boldsymbol{t}})\right] \leq \frac{1}{2},
$$

where $B^{\prime}=\mathcal{T}\left(\operatorname{Dec}_{\boldsymbol{r}_{a}, \hat{\boldsymbol{r}}},(\boldsymbol{x}, \hat{\boldsymbol{t}})\right)$.

Proof. Fix $\boldsymbol{x}, Z$ and the coins of the simulator to some arbitrary values. Then, $M=\mathcal{J} B^{\prime}(\boldsymbol{x}, \hat{\boldsymbol{t}})$ is an $m \times(\ell+1)$ matrix over $\mathbb{F}$, and therefore its columns span a set $S$ of at most $|\mathbb{F}|^{\ell+1}$ vectors in $\mathbb{F}^{m}$. Since $Y_{0}$ is distributed uniformly over $\mathbb{F}^{m}$ and independently of $M$, it falls in $S$ with probability at most $\frac{|\mathbb{F}|^{\ell+1}}{|\mathbb{F}|^{m}}$ which is upper-bounded by $1 / 2$ since $\ell+1<m$.

Claim 6.6. It holds that $\mathcal{D}_{1} \stackrel{c}{\approx} \mathcal{D}_{3}$.

Proof. The proof proceeds via a hybrid argument. First, we claim that $\mathcal{D}_{1} \stackrel{c}{\approx} \mathcal{D}_{2}$. Indeed, let $\mathcal{C}$ be a distinguisher between the two distributions, we construct an adversary $\mathcal{B}$ against the APRG. On input $\left(Y_{0}, Y_{1}\right)$, the adversary $\mathcal{B}$ samples $\boldsymbol{x} \stackrel{R}{\leftarrow} \mathbb{F}$ and returns $\mathcal{C}\left(Y_{0}, \operatorname{Sim}\left(\boldsymbol{x}, Y_{0} \boldsymbol{x}+Y_{1}\right)\right)$. If $\mathcal{B}$ receives as input a sample $\left(Y_{0}, Y_{1}\right)$ from the APRG then it passes $\mathcal{C}$ a random sample from $\mathcal{D}_{1}$. In turn, if $\left(Y_{0}, Y_{1}\right) \stackrel{R}{\leftarrow} \mathbb{F}^{2 m}$, then it passes $\mathcal{C}$ a random sample from $\mathcal{D}_{2}$. Hence, $\mathcal{B}$ breaks the APRG with the same advantage as $\mathcal{C}$ distinguishes between the two distributions.

Next, it is not hard to verify that $\mathcal{D}_{2}$ is identically distributed to $\mathcal{D}_{3}$ as both $Z$ and $Y_{0} \boldsymbol{x}+Y_{1}$ are uniformly distributed in $\mathbb{F}^{m}$.

By combining Claims 6.5 and 6.6 , we conclude that for $\left(Y_{0}, \boldsymbol{x}, \boldsymbol{r}_{a}, \hat{\boldsymbol{r}}, \hat{\boldsymbol{t}}\right) \stackrel{R}{\leftarrow} \mathcal{D}_{1}$ it holds that:

$$
\operatorname{Pr}\left[Y_{0} \in \operatorname{colSpan} \mathcal{J} B^{\prime}(\boldsymbol{x}, \hat{\boldsymbol{t}})\right] \leq \frac{1}{2},
$$

where $B^{\prime}=\mathcal{T}\left(\operatorname{Dec}_{\boldsymbol{r}_{a}, \hat{\boldsymbol{r}}},(\boldsymbol{x}, \hat{\boldsymbol{t}})\right)$. This means that, when the challenge bit $b$ equals to 1 , the adversary $\mathcal{A}$ outputs 1 with probability larger than $1 / 2-\operatorname{neg}(n)$. Recall that, by Claim 6.4 if $b=0$ the adversary $\mathcal{A}$ wins with probability $1-\operatorname{neg}(n)$. Overall, it follows that $\mathcal{A}$ wins the game with a probability of at least $1 / 2-\operatorname{neg}(n)$, thus breaking the computational privacy of the MPC protocol. 


\section{Homomorphic Encryption}

In this section we show that an encryption scheme with strong multiplicative homomorphism cannot be achieved in the arithmetic model. As explained in the introduction, our attack will rely on a reduction to the Arithmetic Predictability Problem. In Section 7.1, we will define this problem, and show that it can be efficiently solved. (Some of the proofs will be deferred to Sections 7.3 and 7.4). Homomorphic encryption will be defined and attacked in Section 7.2 .

\subsection{Main Tool: Algorithm for the Arithmetic Predictability Problem}

We define the Arithmetic Predictability Problem, denoted by $\operatorname{AP}_{d, \varepsilon, \Delta, p}$, which is parameterized by a degree bound $d \in \mathbb{N}$, proximity parameter $\varepsilon \in(0,1)$, entropy gap $\Delta>0$, and some prime $p$ which defines the field $\mathbb{F}=\mathrm{GF}(p)$.

Definition 7.1 (The Arithmetic Predictability Problem $\operatorname{AP}_{d, \varepsilon, \Delta, p}$ ). Given an arithmetic circuit $P=\left(P_{1}, \ldots, P_{m}\right)$ with $m$ outputs and $n$ inputs $x=\left(x_{1}, \ldots, x_{n}\right)$, and an additional target polynomial $T\left(x_{1}, \ldots, x_{m}\right)$ (represented by an arithmetic circuit) distinguish between the following two cases:

- (Yes case:) There exists a (possibly inefficient) rational function $Q(z)=A(z) / B(z)$, where $A: \mathbb{F}^{m} \rightarrow \mathbb{F}$ and $B: \mathbb{F}^{m} \rightarrow \mathbb{F}$ are non-zero polynomials of degree at most $d$, such that

$$
\underset{\boldsymbol{x} \leftarrow \mathbb{F}^{n}}{\operatorname{Pr}}[Q(P(\boldsymbol{x}))=T(\boldsymbol{x}) \mid B(P(\boldsymbol{x})) \neq 0]=1 .
$$

- (No case:) There exists a probability distribution $\left(\mathbf{P}^{\prime}, \mathbf{T}^{\prime}\right)$ over $\mathbb{F}^{m} \times \mathbb{F}$ such that:

$$
\left(\mathbf{P}^{\prime}, \mathbf{T}^{\prime}\right) \quad \text { is } \varepsilon \text {-close to }(P, T)(\boldsymbol{x}) \quad \text { where } \boldsymbol{x} \stackrel{R}{\leftarrow} \mathbb{F}^{n}
$$

and

$$
\tilde{\mathbf{H}}_{\infty}\left(\mathbf{T}^{\prime} \mid \mathbf{P}^{\prime}\right)>\log p-\Delta .
$$

Recall that the average min-entropy $\tilde{\mathbf{H}}_{\infty}(Y \mid X)$ of a random variable $Y$ given a random variable $X$ measures (in logarithmic scale) the probability of guessing $Y$ given X. (See Section 3.1).

About the parameters. We briefly explain the role of the parameters. Consider, for starters, the case where $Q$ is a polynomial (i.e., the denominator $B$ is taken to be some non-zero scalar), and let us focus on a Yes instance. In this case, $Q$ guesses $T(\mathbf{x})$ given $P(\mathbf{x})$, with probability 1 over $\mathbf{x} \stackrel{R}{\leftarrow} \mathbb{F}^{n}$. So $T\left(U_{n}\right)$ is perfectly predictable given $P\left(U_{n}\right)$. In the more general case, where the denominator of $Q$ is a degree $d$ polynomial, the predictor is allowed to "ignore" some of the inputs $\boldsymbol{x}$ (the ones which zero $B$ ). Hence, larger $d$ weakens the predictability of Yes instances and allows to consider richer predictors.

The parameters $\varepsilon$ and $\Delta$ control the unpredictability of No instances. In the extreme case of $\varepsilon=\Delta=0$, the random variable $T\left(U_{n}\right)$ is completely unpredictable given $P\left(U_{n}\right)$ and it cannot be guessed with probability better than $1 / p$. Larger $\varepsilon, \Delta>0$ weaken this condition and make No instances more predictable 16

\footnotetext{
${ }^{16}$ We remark that the use of two parameters for unpredictability (i.e., $\varepsilon$ and $\Delta$ ), as opposed to a single unpredictability parameter, makes the notion more robust in a way which is crucial for our results.
} 
A binary-version of the above problem (denoted by the Gap-Learning Problem) was studied by $\mathrm{ABX} 08$ in the context of proving lower-bounds for PAC-learning. Indeed, thinking of $(P, T)(\mathbf{x})$ as a joint distribution over $m$-dimensional points $p \in \mathbb{F}^{m}$ and their label $t \in \mathbb{F}$, Yes instances are learnable (by a possibly inefficient learner), while No instances are information-theoretic unlearnable. The AP problem is also closely related to the problem of estimating the entropy of a given distribution (represented by a sampling circuit) which was studied by [GV99] for binary circuits and by [DGRV11] for arithmetic circuits. These binary variants are known to be complete for the class SZK of languages that admit a statistical-zero knowledge proofs [GV99, ABX08], and so they are believed to be intractable.

Solving AP. We say that $\operatorname{AP}_{d, \varepsilon, p, \Delta}$ is efficiently solvable if given an input $(P, T)$ of circuit size $s$ it is possible to determine in time poly $(s, \log p)$ whether $(P, T)$ is a Yes instance or a No instance with error probability of $1 / 3$. We will show that, when $\mathbb{F}$ is sufficiently large, the problem can be solved efficiently. Recall that $A$ is a strictly arithmetic circuit if it does not contains any bitsample gates, sample gates, zerocheck gates, or divide gates. (Recall that a generalized arithmetic circuit may use such gates.) We will always assume that the target polynomial $T$ is a strictly arithmetic circuit as this will be the case in our attack. (Specifically, for our attack, the target polynomial is simply a projection of the first variable $T(x)=x_{1}$.)

Theorem 7.2. The problem $\mathrm{AP}_{d, \varepsilon, p, \Delta}$ can be efficiently solved in the following cases.

1. The inputs $P: \mathbb{F}^{n} \rightarrow \mathbb{F}^{m}$ and $T: \mathbb{F}^{n} \rightarrow \mathbb{F}$ are strictly arithmetic circuits which compute degree $d$ polynomials, $\varepsilon<\frac{1}{2}$ and $p>\frac{(n+1) d^{n+1}\left(1+2^{\Delta}\right)}{(1 / 2-\varepsilon)^{2}}$.

2. The input $P: \mathbb{F}^{n} \rightarrow \mathbb{F}^{m}$ is an s-size (generalized) arithmetic circuit, the input $T$ is an s-size strictly arithmetic circuit, $\varepsilon \leq 0.199, p \geq(d+s)^{2 s} \cdot 2^{3 s+\Delta}$, and $s$ is sufficiently large ${ }^{17}$

In the second part $P$ is allowed to use internal random gates (which sample bits or field elements). In such a case, we assume that the probability distributions of Equations (9) and (10) are defined over the internal randomness of $P$, in addition to the random choice of $x \stackrel{R}{\leftarrow} \mathbb{F}^{n}$.

Proof outline. The first part is proved via the following simple algorithm: Choose a random point $\boldsymbol{x} \stackrel{R}{\leftarrow} \mathbb{F}^{n}$ and check if the rows of the Jacobian $\mathcal{J} P(\boldsymbol{x}) \in \mathbb{F}^{m \times n}$ span the gradient $\partial T(\boldsymbol{x}) \in \mathbb{F}^{n}$ of the target polynomial $T(x)$. The analysis follows the techniques of [DGW07] and is differed to Section 7.3. For the second part (which will be useful for our attack) we show how to map the input $(P, T)$ into a strictly arithmetic circuit $\left(P^{\prime}, T\right)$ via a sequence of (randomized) Karp reductions. The proof then follows by applying the first part of the theorem. (See Section 7.4.) We note that the second part of the theorem extends to the case where $\varepsilon \leq 1 / 5-\alpha$ for some inverse polynomial $\alpha$ at the expense of letting $p=\Omega\left(\frac{\exp \left(2 s^{3}+s \log (d+1)+\Delta\right)}{\alpha^{2}}\right)$.

Remark 7.3. As we will later see in order to attack arithmetic multiplicative encryption, it suffices to solve a very special case of $\mathrm{AP}_{d, \varepsilon, p, \Delta}$ where $\Delta=0, \varepsilon$ is negligible (or even taken to be zero), $d=2^{\text {poly(s) }}$ and $p$ is a huge (exponentially large) prime. However, this should be done with respect to a generalized arithmetic circuit $P$ which may use division gates, zero-testing gates and bit-sample

\footnotetext{
${ }^{17}$ Note that $s$ implicitly upper-bounds the input and output lengths ( $n$ and $m$ ) and therefore we can state the result without putting an explicit bound on $n$ and $m$.
} 
gates. The only way we know how to solve this problem is via a reduction to the strictly arithmetic case with a more general setting of parameters $d, \Delta, \varepsilon>0$.

\subsection{Main Results: Attacking Strongly Homomorphic Encryption}

Recall that an arithmetic encryption scheme is defined by a triple (KGen, Enc, Dec) where KGen = $\left\{\mathrm{KGen}_{n}\right\}, \mathrm{Enc}=\left\{\mathrm{Enc}_{n}\right\}$ and $\mathrm{Dec}=\left\{\mathrm{Dec}_{n}\right\}$ are uniform sequences of polynomial sized arithmetic circuits. (See Definition 4.2.) We assume that $\mathrm{KGen}_{n}$ outputs an additional evaluation key ek and that security holds even when the adversary is given ek at the first step of the security game. We further assume that the scheme satisfies the following notion of homomorphic evaluation (related notions have appeared in [IKO05, Gen09, BL13]).

Definition 7.4 (Multiplicative Homomorphic Evaluator). An $\varepsilon$-strong multiplicative homomorphic evaluator for an encryption scheme (KGen, Enc, Dec) is a uniform sequence of polynomial sized (randomized) arithmetic circuits $\mathrm{Eval}=\left\{\mathrm{Eval}_{n}\right\}$ that takes as input a ciphertext $c$, a message $y$ and an evaluation-key ek. It should satisfy the following conditions for all fields $\mathbb{F}$ and for all but a negligible fraction of the keys (pk, sk, ek) $\stackrel{R}{\leftarrow} \mathrm{KGen}_{n}^{\mathbb{F}}$ :

- (Correctness) For every pair of messages $x \in \mathbb{F}$ and $y \in \mathbb{F}$, the ciphertext $c=\operatorname{Eval}_{n}^{\mathbb{F}}\left(\right.$ ek, $\left.\operatorname{Enc}_{n}^{\mathbb{F}}(x, \mathrm{pk}), y\right)$ decrypts correctly (with probability 1 ) to $x \cdot y$, i.e.,

$$
\operatorname{Pr}\left[\operatorname{Dec}_{n}(c, \mathrm{sk})=x y\right]=1 .
$$

- (Strong Homomorphism) For every pair of messages $x \in \mathbb{F}$ and $y \in \mathbb{F}$, the random variables

$$
\left(c, \operatorname{Eval}_{n}^{\mathbb{F}}(c, y, \mathrm{ek})\right) \text { and }\left(c, \operatorname{Enc}_{n}^{\mathbb{F}}((x \cdot y), \mathrm{pk})\right), \quad \text { where } c \stackrel{R}{\leftarrow} \operatorname{Enc}_{n}^{\mathbb{F}}(x, \mathrm{pk}),
$$

are $\varepsilon(n)$-close in statistical distance.

We say that Eval is $\varepsilon$-weak multiplicative homomorphic evaluator if Strong Homomorphism is replaced with the following property

- (Weak Homomorphism) For every pair of messages $x \in \mathbb{F}$ and $y \in \mathbb{F}$, the random variables

$$
\mathrm{Eval}_{n}^{\mathbb{F}}\left(\mathrm{Enc}_{n}^{\mathbb{F}}(\mathrm{pk}, x), y, \mathrm{ek}\right) \quad \text { and } \quad \mathrm{Enc}_{n}^{\mathbb{F}}((x \cdot y), \mathrm{pk}) \text {, }
$$

are $\varepsilon(n)$-close in statistical distance.

We rule out arithmetic commitments with strong multiplicative homomorphism even in the weakest setting of symmetric encryption with one-time security. In this setting, we may assume, without loss of generality, that the scheme is perfectly correct ${ }^{18}$ In contrast, we will later show (Section 10) that weak multiplicative homomorphism can be achieved in the arithmetic model based on the RLC assumption.

\footnotetext{
${ }^{18}$ First, observe that without loss of generality the decryption algorithm is deterministic (e.g., by letting the encryption output the randomness needed for decryption as part of the ciphertext). Next, note that any scheme with negligible decryption error of $\varepsilon(n)$ can be turned into a perfectly correct scheme. This is done by letting the encryption algorithm check if the resulting ciphertext decrypts correctly, and, in case of an error, replace the ciphertext with the un-encrypted message (together with a special "no encryption" flag). It is not hard to see that this transformation incurs only a negligible loss (of $\varepsilon$ ) in the security.
} 
Theorem 7.5. For every constant $\varepsilon \leq 0.199$, there is no $\varepsilon$-strong one-time secure symmetric encryption scheme in the arithmetic model.

Proof. Let $s(n)$ be an upper-bound on the circuit size of the homomorphic evaluator Eval ${ }_{n}$ and on the circuit size of the decryption algorithm. We will show that in order to attack the scheme it suffices to solve the $\operatorname{AP}_{d, \varepsilon, p, \Delta}$ for $d=(s+1) \cdot 2^{s}, \Delta=0, \varepsilon=0.199$ and sufficiently large $p$. Specifically, the attacker $\mathcal{A}$ does the following:

- $\mathcal{A}$ receives $1^{n}$, chooses a prime $p \in\left(2^{7 s^{2}}, 2 \cdot 2^{7 s^{2}}\right)$ and sends some implementation of the field $\mathbb{F}=\mathrm{GF}(p)$ to the challenger.

- Given an evaluation key ek $\stackrel{R}{\leftarrow} \mathrm{KGen}_{n}^{\mathbb{F}}$, the adversary chooses a pair of messages $\left(x_{0}=0, x_{1}=\right.$ 1) and sends them to the challenger.

- Given a ciphertext $c$ (which encrypts either $x_{0}$ or $x_{1}$ ), the adversary defines a randomized arithmetic circuit $P_{\mathrm{ek}, c}(x)=\mathrm{Eval}_{n}^{\mathbb{F}}(c, x, \mathrm{ek})$ and a strictly arithmetic circuit $T(x)=x$. Then, it uses part 2 of Theorem 7.2 to classify $(P, T)$ as a Yes instance or a No instance of $\mathrm{AP}_{d=(s+1) \cdot 2^{s}, \varepsilon, p, \Delta=0}$. If the answer is "Yes", $\mathcal{A}$ outputs 1 ; otherwise, it outputs 0.

Fix the keys (sk, pk, ek) that were chosen by $\mathrm{KGen}_{n}^{\mathbb{F}}$ and let us condition on the event that these keys are "good" in the sense that they satisfy the Correctness and Strong Homomorphism properties of Definition 7.4. (Recall that this event happens with all but negligible probability.) To analyze the success probability it suffices to show that if $c$ is an encryption of 1 then $\left(P_{\mathrm{ek}, c}, T\right)$ is a Yes instance and if $c$ is an encryption of 0 then $\left(P_{\mathrm{ek}, c}, T\right)$ is a No instance.

We begin with the case where $c$ is an encryption of 1 , and, correspondingly, $P_{\mathrm{ek}, c}(x)$ outputs an encryption of $x \cdot 1=x$. By perfect correctness, the decryption algorithm $\operatorname{Dec}_{\mathrm{sk}}(z)=\operatorname{Dec}(z, \mathrm{sk})$ satisfies $\operatorname{Dec}_{\mathrm{sk}}\left(\left(P_{\mathrm{ek}, c}(\boldsymbol{x})\right)=T(\boldsymbol{x})\right.$ for every $\boldsymbol{x}$. Recall that, by Proposition 3.11, there exists a rational function $\frac{A}{B}$ which is computed by a zerocheck-free circuit of size $s$, and a degree $s 2^{s}$ polynomial $G$ such that $\frac{A}{B}(\boldsymbol{z})=\operatorname{Dec}_{\text {sk }}(\boldsymbol{z})$ for every $\boldsymbol{z}$ which is not a root of $G$. Consider the rational function $Q(z)=\frac{A(z) G(z)}{B(z) G(z)}$. This function certifies that $\left(P_{\mathrm{ek}, c}, T\right)$ is a Yes instance, since $Q\left(P_{\text {ek }, c}(\boldsymbol{x})\right)=T(\boldsymbol{x})$ for every $\boldsymbol{x}$ which satisfies $B(P(\boldsymbol{x})) G(P(\boldsymbol{x})) \neq 0$. Finally, observe that the degree of the denominator and nominator of $Q$ is upper-bounded by $(s+1) \cdot 2^{s}$.

Next, we claim that if $c$ is an encryption of 0 then $\left(P_{\mathrm{ek}, c}, T\right)$ is a No instance. Indeed, in this case $P_{\text {ek,c }}(x)$ outputs the ciphertext $x \cdot 0=0$, and, since the homomorphism is strong, we have that for a every good keys pk and ek, and every message $\boldsymbol{x}$, the random variables

$$
\left(c, \operatorname{Eval}_{n}^{\mathbb{F}}(\mathrm{ek}, c, \boldsymbol{x})\right) \quad \text { and } \quad\left(c, \operatorname{Enc}_{n}^{\mathbb{F}}(\mathrm{pk}, 0)\right)
$$

are $\varepsilon(n)$-close. Letting $\left(\mathbf{P}^{\prime}, \mathbf{T}^{\prime}\right)=\left(\operatorname{Enc}_{n}^{\mathbb{F}}(\mathrm{pk}, 0), T(\boldsymbol{x})\right)$ where $\boldsymbol{x} \stackrel{R}{\leftarrow} \mathbb{F}$, we conclude that

$$
\left(P_{\mathrm{ek}, c}(\boldsymbol{x}), T(\boldsymbol{x})\right) \quad \text { is } \varepsilon \text {-close to } \quad\left(\mathbf{P}^{\prime}, \mathbf{T}^{\prime}\right) .
$$

Since $\mathbf{T}^{\prime}=T(\boldsymbol{x})$ is uniform and statistically independent of $\mathbf{P}^{\prime}=\mathrm{Enc}_{n}^{\mathbb{F}}(\mathrm{pk}, 0)$, it follows that $\tilde{\mathbf{H}}_{\infty}\left(\mathbf{T}^{\prime} \mid \mathbf{P}^{\prime}\right)=\log p$, and so $\left(P_{\text {ek,c }}, T\right)$ is a No instance. The theorem follows.

Remarks on Theorem 7.5 . 
- For positive applications, one would typically strive for strong homomorphic encryption with negligible error parameter $\varepsilon$. Theorem 7.5 therefore provides a strong negative result as it applies even to a constant $\varepsilon \leq 0.199$. (Similarly to Theorem 7.2 , the lower-bound holds for any value of $\varepsilon=\frac{1}{5}-\delta(n)$ where $\delta$ is an arbitrary inverse polynomial.)

- In the public-key setting, it is possible to extend the theorem to the case where the strong homomorphism property (as defined in Definition 7.4) applies only to a noticeable fraction of the evaluation/public-keys (ek, pk). Indeed, in this case the attack $\mathcal{A}$ described in the proof of Theorem 7.5 succeeds over a noticeable fraction of the keys. The scheme can now be broken by applying $\mathcal{A}$ over "vulnerable" keys and using a random guess over all other keys (for which the strong homomorphism does not hold). This strategy can be efficiently implemented since one can efficiently test (with high probability) whether the keys chosen by the challenger are vulnerable to $\mathcal{A}$ or not. (Just run the attack several times on a pair of "dummy ciphertexts" for whose plaintexts are known and check whether it succeeds.) A similar extension holds for the case of CPA-secure symmetric encryption as in this case the encryption oracle can be used to test if the given evaluation-key ek is vulnerable.

- Finally, we note that the attack generalizes to the case where the decryption algorithm can be written as a possibly-inefficient polynomial of degree $2^{\text {poly }(n)}$. This extension can be used to handle restricted forms of arithmetic commitment schemes with strong multiplicative homomorphism which support inefficient low-degree decryption.

\subsection{Proof of Theorem 7.2 ; part 1}

The idea is to output Yes if the rows of the Jacobian $\mathcal{J} P: \mathbb{F}^{n} \rightarrow \mathbb{F}^{m \times n}$ span the gradient $\partial T(x)$ : $\mathbb{F}^{n} \rightarrow \mathbb{F}^{n}$ of the target polynomial $T(x)$, where the underlying field is the field of rational functions $\mathbb{F}(x)$ and $\partial T(x)$ is viewed as a row vector. As observed by [DGW07] (see also [Kay09, Corollary 3]) this can checked efficiently (with small error) via the following randomized algorithm.

The algorithm $\mathcal{A}$ : Choose a random vector $\mathrm{x} \stackrel{R}{\leftarrow} \mathbb{F}^{n}$ and output "Yes" if and only if the rows of the matrix $\mathcal{J} P(\mathbf{x}) \in \mathbb{F}^{m \times n}$ span (over $\mathbb{F}$ ) the row vector $\partial T(\mathbf{x}) \in \mathbb{F}^{n}$.

Let us analyze the algorithm $\mathcal{A}$ beginning with the case of a Yes instance.

Claim 7.6. If the input is a Yes instance then the algorithm outputs Yes with probability $1-\frac{2 d^{2}}{p}>\frac{2}{3}$.

Proof. Assume that the input is a Yes instance. Namely, for some rational function $Q\left(z_{1}, \ldots, z_{m}\right)=$ $A\left(\left(z_{1}, \ldots, z_{m}\right)\right) / B\left(\left(z_{1}, \ldots, z_{m}\right)\right)$ it holds that

$$
Q(P(\boldsymbol{x}))=T(\boldsymbol{x})
$$

for every $\boldsymbol{x}$ which does not zero $B(P(\boldsymbol{x}))$. Note that the degrees of the denominator and nominator of the LHS are upper-bounded by $d^{2}$ and the degree of the polynomial of the RHS is upper-bounded by $d$. Since $\mathbb{F}$ is sufficiently large, it follows (by the Schwartz-Zippel lemma) that the functions $Q(P(x))$ and $T(x)$ are equivalent as polynomials. Then, by the chain rule, we have that

$$
\partial_{z} Q(P(x)) \cdot \mathcal{J}_{x} P(x) \equiv \partial_{x} T(x)
$$


where $\partial_{z} Q(P(x))$ and $\partial_{x} T(x)$ are treated as row vectors. Hence, if the vector $\mathbf{x} \in \mathbb{F}^{n}$ does not zero the denominator of $\partial_{z} Q(P(x))$, the test will pass and the algorithm will output "Yes".

We show that the denominator of $\partial_{z} Q(P(x))$ is zeroed with probability at most $2 d^{2} /|\mathbb{F}|$. Recall that $Q(z)=A(z) / B(z)$ and so the denominator of $\partial_{z} Q(P(x))$ is $B^{2}(P(x))$ which is a polynomial of degree at most $2 d^{2}$. (Since $d$ upper-bounds the degree of $B$ and the degree of the polynomials $P$.) Hence, a random $\mathbf{x} \underset{\leftarrow}{\leftarrow} \mathbb{F}^{n}$ will zero $\partial_{z} Q(P(x))$ with probability at most $2 d^{2} /|\mathbb{F}|$ which, for our choice of parameters, is (much) smaller than $1 / 3$.

We move on to the case of a No instance. We will need the following fact from [DGW09, originally proved over the field of rational numbers by [ER93].

Fact 7.7 (Theorems 3.1 and 3.3 in DGW09]). Let $\mathbb{F}=\mathrm{GF}(p)$ and let $M=\left(M_{1}, \ldots, M_{m}\right)$ be an $m$-tuple of degree d polynomials in $\mathbb{F}\left[x_{1}, \ldots, x_{n}\right]$ where $p$ is a prime larger than $D \stackrel{\text { def }}{=}(n+1) d^{n}$. Then, the rows of the Jacobian $\mathcal{J} M$ are linearly dependent (over the field of rational functions) if and only if there exists a polynomial $Z \in \mathbb{F}\left[z_{1}, \ldots, z_{m}\right]$ such that $Z\left(M_{1}(x), \ldots, M_{m}(x)\right) \equiv 0$. Furthermore, in the "only if" direction $Z$ is guaranteed to be of degree at most $D$.

We remark that the "if" direction holds even if $p$ is small. The polynomial $Z$ is referred to as the annihilating polynomial of $M$ and its existence corresponds to the classical notion of algebraic dependence.

We begin by showing that, in the case of No instance, the Jacobian $\mathcal{J} P$ does not span the gradient $\partial T$ of the target polynomial over the field of rational functions $\mathbb{F}(x)$.

Claim 7.8. If the input is a No instance, then $\partial T \notin \operatorname{span}(\mathcal{J} P)$.

Proof. Assume towards a contradiction that $\partial T \in \operatorname{span}(\mathcal{J} P)$. Without loss of generality, we assume that the rows of $\mathcal{J} P$ are linearly independent. (Otherwise, take some minimal subset $P^{\prime}$ of the polynomials in $P$ whose jacobian spans $\mathcal{J} P$; Observe that if $(P, T)$ is a No instance then so is $\left(P^{\prime}, T\right)$ for any subset of the polynomials $P^{\prime} \subseteq P$.)

By Fact 7.7, there exists an annealing polynomial $Z\left(z_{1}, \ldots, z_{m}, t\right)$ of degree $D=(n+1) d^{n}$ such that $Z(T, P) \equiv 0$. Furthermore, since the rows of $\mathcal{J} P$ are linearly independent, by Fact 7.7 , the polynomials $P$ have no annealing polynomial. It follows that $Z$ can be written as

$$
Z(z, t)=\sum_{i=1}^{D} t^{i} \cdot Z_{i}(z), \quad \text { where the polynomial } Z_{i}(P(x)) \not \equiv 0 \text { for some } i .
$$

Since $(P, T)$ is assumed to be a No instance, there exists a distribution $\left(\mathbf{P}, \mathbf{T}^{\prime}\right)$ such that (1) $\left(\mathbf{P}, \mathbf{T}^{\prime}\right)$ is $\varepsilon$-close to $(P, T)\left(U_{n}\right)$ and $(2) \tilde{\mathbf{H}}_{\infty}\left(\mathbf{T}^{\prime} \mid \mathbf{P}\right)>\log p-\Delta$. We will use (2) to derive a contradiction to (1). That is, we describe an (inefficient) distinguisher $\mathcal{A}$ for $\left(\mathbf{P}^{\prime}, \mathbf{T}^{\prime}\right)$ and $(P, T)\left(U_{n}\right)$.

Given a vector $\left(z=\left(\mathbf{z}_{1}, \ldots, \mathbf{z}_{m}\right), \mathbf{t}\right) \in \mathbb{F}^{m+1}$, our distinguisher $\mathcal{A}$ accepts the input if and only if $Z\left(\left(\mathbf{z}_{1}, \ldots, \mathbf{z}_{m}\right), \mathbf{t}\right)=0$. By definition, the test accepts every value $(z, t)$ in the image of $(P, T)(x)$, therefore,

$$
\operatorname{Pr}_{\mathbf{x}}[\mathcal{A}(P(\mathbf{x}), T(\mathbf{x}))=1]=1 .
$$

We analyze the acceptance probability of $\left(\mathbf{P}^{\prime}, \mathbf{T}^{\prime}\right)$. For a fixed $\boldsymbol{z} \in \mathbb{F}^{m}$, let $L_{\boldsymbol{z}}$ denote the set of $t \in \mathbb{F}$ for which the distinguisher accepts the value $(\boldsymbol{z}, t)$. Namely,

$$
L_{\boldsymbol{z}} \stackrel{\text { def }}{=}\{t \in \mathbb{F}: Z(\boldsymbol{z}, t)=1\} .
$$


First observe that

$$
\operatorname{Pr}_{\boldsymbol{z} \leftarrow}^{\operatorname{Rr} P\left(U_{n}\right)}\left[\left|L_{\boldsymbol{z}}\right|<D d\right]>1-\frac{D d}{p} .
$$

Indeed, let $i$ be the maximal integer for which $Z_{i}(P(x))$ is a non-zero polynomial, as promised by Equation (12). As a polynomial in $x$, the degree of $Z_{i}(P(x))$ is at most $(D-i) \cdot d<D d$, hence, by the Schwartz-Zippel lemma, $\operatorname{Pr}_{\mathbf{x}}\left[Z_{i}(P(\mathbf{x}))=0\right]<D d /|\mathbb{F}|$. For any fixed $\mathbf{x}$ for which $Z_{i}(P(\mathbf{x})) \neq 0$, the residual polynomial $Z_{\boldsymbol{x}}(t)=Z(P(\mathbf{x}), t)$ is a non-zero polynomial of degree at most $i \cdot d<D d$ and so it has at most $D d$ roots and Eq. 13 follows.

Since the marginals $\mathbf{P}$ and $P\left(U_{n}\right)$ are $\varepsilon$-close, Eq. (13) implies that

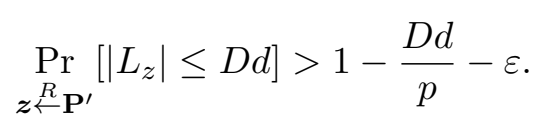

Recall that $\tilde{\mathbf{H}}_{\infty}\left(\mathbf{T}^{\prime} \mid \mathbf{P}^{\prime}\right)>\log p-\Delta$, and so by Markov's inequality (Fact 3.1 (item 1), we have that for every $\delta>0$

$$
\underset{\boldsymbol{z} \stackrel{R}{\leftarrow} \mathbf{P}^{\prime}}{\operatorname{Pr}}\left[\mathbf{H}_{\infty}\left(\mathbf{T}^{\prime} \mid \mathbf{P}^{\prime}=\boldsymbol{z}\right)>\log p-\Delta-\log (1 / \delta)\right]>1-\delta .
$$

Call $\boldsymbol{z}$ good if both $\left|L_{z}\right| \leq D d$ and $\mathbf{H}_{\infty}\left(\mathbf{T}^{\prime} \mid \mathbf{P}^{\prime}=\boldsymbol{z}\right)>\log p-\Delta-\log (1 / \delta)$. Combining Equations (14) and 15 , and applying a union-bound, it follows that a random $z \stackrel{R}{\leftarrow} \mathbf{P}^{\prime}$ is good with probability $1-\delta-\varepsilon-\frac{D d}{p}$. Finally, observe that for a good $z$ we have that

$$
\operatorname{Pr}\left[\mathbf{T}^{\prime} \in L_{\boldsymbol{z}} \mid \mathbf{P}^{\prime}=\boldsymbol{z}\right]<D d \cdot 2^{-\log p-\Delta-\log (1 / \delta)} \leq \frac{D d 2^{\Delta}}{\delta p}
$$

Hence, the distinguisher $\mathcal{A}$ accepts $\left(\mathbf{P}^{\prime}, \mathbf{T}^{\prime}\right)$ with probability at most $\left(\delta+\frac{D d}{p}+\varepsilon\right)+\frac{D d 2^{\Delta}}{\delta p}$. Overall, the distinguishing advantage is $1-\left(\delta+\frac{D d}{p}+\varepsilon+\frac{D d 2^{\Delta}}{\delta p}\right)$. Taking $\delta=(1-2 \varepsilon) / 2$ and plugging in $p>\frac{D d\left(1+2^{\Delta}\right)}{(1 / 2-\varepsilon)^{2}}$ we obtain a distinguishing advantage larger than $\varepsilon$.

Finally, it remains to show that if $\partial T \notin \operatorname{span}(\mathcal{J} P)$ then for a random $\mathbf{x} \stackrel{R}{\leftarrow} \mathbb{F}^{n}$, the matrix $\partial T(\mathbf{x}) \notin \operatorname{span}(\mathcal{J} P)(\mathbf{x})$. This follows from the following standard claim.

Claim 7.9. Let $M$ be an $m \times n$ matrix of degree $d$ polynomials in $\mathbb{F}\left[x_{1}, \ldots, x_{n}\right]$ and let $v$ be a vector of $n$ polynomials of degree $d$ over $\mathbb{F}\left[x_{1}, \ldots, x_{n}\right]$. Then, if the rows of the Jacobian $M$ do not span $v$ over the field of rational functions $\mathbb{F}(x)$, then $\operatorname{Pr}_{\mathbf{x}}[v(\mathbf{x}) \in \operatorname{span}(M(\mathbf{x}))] \leq d n /|\mathbb{F}|$.

Proof. Let $M^{\prime}$ be the matrix obtained by taking a subset of the rows of $M$ that forms a basis for $M$. Since the matrix $M^{\prime}$ has full rank and since it does not span $v$, the number of rows $m^{\prime} \leq m$ of $M^{\prime}$ is strictly smaller than the number of columns $n$. We define an $m^{\prime} \times\left(m^{\prime}+1\right)$ matrix $M^{\prime \prime}$ by removing an arbitrary set of $n-m^{\prime}-1$ columns from $M^{\prime}$, and define $v^{\prime}$ be removing the corresponding entries of $v$. Observe that

$$
\underset{\mathbf{x}}{\operatorname{Pr}}[v(\mathbf{x}) \in \operatorname{span}(M(\mathbf{x}))]=\operatorname{Pr}_{\mathbf{x}}\left[v(\mathbf{x}) \in \operatorname{span}\left(M^{\prime}(\mathbf{x})\right)\right] \leq \operatorname{Pr}_{\mathbf{x}}\left[v^{\prime}(\mathbf{x}) \in \operatorname{span}\left(M^{\prime \prime}(\mathbf{x})\right)\right] .
$$

Hence, it suffices to show that the square matrix $T=\left(\begin{array}{c}M_{v}^{\prime \prime} \\ v\end{array}\right)$ of polynomials is likely to be nondegenerate when evaluated on a random point $\mathbf{x}$. Indeed,

$$
\underset{\mathbf{x}}{\operatorname{Pr}}[\operatorname{det}(T(\mathbf{x}))=0]=\underset{\mathbf{x}}{\operatorname{Pr}}[\operatorname{det}(T)(\mathbf{x})=0] \leq d^{n} /|\mathbb{F}|
$$


where $\operatorname{det}(T)$ is the polynomial $P(x)$ that corresponds to the determinant of $T$ computed over the the field of rational functions. The last inequality follows by Schwartz-Zippel, noting that $P$ is a non-zero polynomial (since $T$ is non-degenerate over $\mathbb{F}(x)$ ) of degree at most $n d$.

Combining the last two claim, we conclude that No instances are accepted with probability at most $1 / 3$. This completes the proof of Theorem 7.2 .

\subsection{Proof of Theorem 7.2 ; part 2}

The proof follows by a sequence of Karp reductions from generalized Arithmetic Circuits to strictly arithmetic circuits.

\subsubsection{Removing random gates}

We begin by describing how to remove sample gates (which sample uniform field elements) and bitsample gates (which sample a uniform 0-1 values). Handling the first type is easy: Just view the sample gates as additional input gates. Formally, let $\ell \leq s$ denote the number of sample gates, and for $x \in \mathbb{F}^{n}$ and $R \in \mathbb{F}^{\ell}$, let $P(x ; R)$ denote the outcome of $P(x)$ when the sample gates are fixed to the value $R$. Then, the circuit $P^{\prime}(x, R)=P(x ; R)$ has no sample gates. Furthermore, it is not hard to verify that if $(P, T)$ is a Yes instance (resp., No instance) then so is $\left(P^{\prime}, T^{\prime}\right)$ where $T^{\prime}(x, R)=T(x)$.

The case of bitsample gates is slightly more complicated. Let $T: \mathbb{F}^{n} \rightarrow \mathbb{F}$ be an $s$-size strictly arithmetic circuit and let $P: \mathbb{F}^{n} \rightarrow \mathbb{F}^{m}$ is an $s$-size arithmetic circuit that uses $\ell<s$ bitsample gates. For a string $\rho \in\{0,1\}^{\ell}$ let $P_{\rho}$ denote the arithmetic circuit obtained by fixing the value of the bitsample gates of $P$ to $\rho$.

Lemma 7.10 (Removing bitsample gates). For every $\alpha>0$ the following hold:

- If $(P, T)$ is a Yes instance of $\mathrm{AP}_{d, \varepsilon, p, \Delta}$ then, for every $\rho,\left(P_{\rho}, T\right)$ is a Yes instance of $\mathrm{AP}_{d, \varepsilon^{\prime}, p, \Delta^{\prime}}$.

- If $(P, T)$ is a No instance of $\operatorname{AP}_{d, \varepsilon, p, \Delta}$, then with probability $\alpha$ over $\rho \stackrel{R}{\leftarrow}\{0,1\}^{\ell}$, the pair $\left(P_{\rho}, T\right)$ is a No instance of $\mathrm{AP}_{d, \varepsilon^{\prime}, p, \Delta^{\prime}}$

where $\varepsilon^{\prime}=\frac{2 \varepsilon}{1-\varepsilon-3 \alpha / 2}$ and $\Delta^{\prime}=\Delta+s+\log (1 / \alpha)+1$.

Proof. The first item holds by definition. We will prove that, with high probability, a No instance is mapped to a No instance. Let $R$ be the uniform distribution over $\{0,1\}^{\ell}$ strings. Since $(P, T)$ is a No instance, there exists a distribution $(\mathbf{P}, \mathbf{T})$ which is $\varepsilon$-close to $\left(P\left(U_{n}, R\right), T\left(U_{n}\right)\right)$ and for which $\tilde{\mathbf{H}}_{\infty}\left(\mathbf{T}^{\prime} \mid \mathbf{P}^{\prime}\right)>\log p-\Delta$. Consider the joint distribution $\left(P\left(U_{n}, R\right), T\left(U_{n}\right), R\right)$ and observe that we can define a new random variable $\mathbf{R}^{\prime}$ which is jointly distributed with $\left(\mathbf{T}^{\prime}, \mathbf{P}^{\prime}\right)$ such that the distributions

$$
\left(P\left(U_{n}, R\right), T\left(U_{n}\right), R\right) \quad \text { and } \quad\left(\mathbf{T}^{\prime}, \mathbf{P}^{\prime}, \mathbf{R}^{\prime}\right) \quad \text { are } \varepsilon-\text { close. }
$$

Specifically, let $\mathbf{R}^{\prime}=f\left(\mathbf{T}^{\prime}, \mathbf{P}^{\prime}\right)$ where $f(p, t)$ is the randomized mapping that given $(p, t) \in \mathbb{F}^{m} \times \mathbb{F}$ uniformly samples $(r, x) \stackrel{R}{\leftarrow}(P, T)^{-1}(p, t)$ and outputs $r$. Observe that

$$
\left(P\left(U_{n}, R\right), T\left(U_{n}\right), R\right) \equiv\left(P\left(U_{n}, R\right), T\left(U_{n}\right), f\left(P\left(U_{n}, R\right), T\left(U_{n}\right)\right)\right),
$$


and, by definition,

$$
\left(\mathbf{T}^{\prime}, \mathbf{P}^{\prime}, \mathbf{R}^{\prime}\right) \equiv\left(\mathbf{T}^{\prime}, \mathbf{P}^{\prime}, f\left(\mathbf{T}^{\prime}, \mathbf{P}^{\prime}\right)\right)
$$

Hence, Eq. 16 follows from the fact that $(\mathbf{P}, \mathbf{T})$ and $\left(P\left(U_{n}, R\right), T\left(U_{n}\right)\right)$ are $\varepsilon$-close.

We will use the following notation. For a jointly distributed $(X, Y)$, let $X_{Y=y}$ denote the distribution of $X$ conditioned on the event $Y=y$. Let $\alpha_{1}, \alpha_{2}>0$. Call $\rho \in\{0,1\}^{\ell}$ good if it satisfies the following conditions (Equations (17) and (18)):

$$
\left(P\left(U_{n}, R\right), T\left(U_{n}\right)\right)_{R=\rho} \quad \text { and } \quad\left(\mathbf{T}^{\prime}, \mathbf{P}^{\prime}\right)_{\mathbf{R}^{\prime}=\rho} \quad \text { are }\left(2 \varepsilon / \alpha_{1}\right)-\text { close }
$$

and

$$
\tilde{\mathbf{H}}_{\infty}\left(\mathbf{T}_{\mathbf{R}^{\prime}=\rho}^{\prime} \mid \mathbf{P}_{\mathbf{R}^{\prime}=\rho}^{\prime}\right)>\log p-\Delta-s-\log \left(1 / \alpha_{2}\right) .
$$

Note that if $\rho$ is good then $\left(P_{\rho}, T\right)$ is indeed a No instance of $\mathrm{AP}_{d, \varepsilon^{\prime}, p, \Delta^{\prime}}$. We begin by showing that a large fraction of $\rho \in\{0,1\}^{\ell}$ satisfy Eq. (17). We will need the following simple fact.

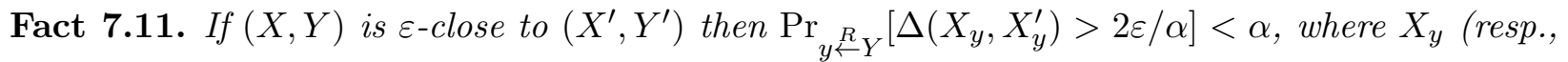
$X_{y}^{\prime}$ ) denote the distribution of $X$ (resp. $\left.X^{\prime}\right)$ conditioned on the event $Y=y$ (resp., $Y^{\prime}=y$ ).

Proof. By Markov's inequality, it suffices to prove that

$$
\underset{y \leftarrow R}{\mathbb{R}}\left[\Delta\left(X_{y}, X_{y}^{\prime}\right)\right] \leq 2 \varepsilon
$$

For every $y$ and distinguisher $A$, let $\alpha(y, A)=\operatorname{Pr}\left[A\left(X_{y}\right)\right]-\operatorname{Pr}\left[A\left(X_{y}^{\prime}\right)\right]$, where $\operatorname{Pr}[A(x)]$ abbreviates $\operatorname{Pr}[A(x)=1]$. Let $A_{y}$ be a distinguisher that maximizes, over all distinguishers $A$, the quantity $\alpha(y, A)$ and let $\alpha_{y}=\alpha(y, A)$. Note that $\alpha_{y}$ is the statistical distance between $X_{y}$ and $X_{y}^{\prime}$. We can write

$$
\begin{aligned}
& \underset{y \stackrel{R}{\leftarrow} Y}{\mathbb{E}}\left[\operatorname{Pr}\left[A_{y}\left(X_{y}\right)\right]-\operatorname{Pr}\left[A_{y}\left(X_{y}^{\prime}\right)\right]\right]=\underset{y \leftarrow \text { R }}{\mathbb{E}}\left[\operatorname{Pr}\left[A_{y}\left(X_{y}\right)\right]\right]-\underset{y \stackrel{R}{\leftarrow} Y}{\mathbb{E}}\left[\operatorname{Pr}\left[A_{y}\left(X_{y}^{\prime}\right)\right]\right] \\
& =\underset{y \leftarrow}{\mathbb{R}}\left[\operatorname{Pr}\left[A_{y}\left(X_{y}\right)\right]\right]-\left(\underset{y \stackrel{R}{\leftarrow} Y^{\prime}}{\mathbb{E}}\left[\operatorname{Pr}\left[A_{y}\left(X_{y}^{\prime}\right)\right]\right]-\underset{y \stackrel{R}{\leftarrow} Y^{\prime}}{\mathbb{E}}\left[\operatorname{Pr}\left[A_{y}\left(X_{y}^{\prime}\right)\right]\right]\right) \\
& -\underset{y \stackrel{R}{R} Y}{\mathbb{E}}\left[\operatorname{Pr}\left[A_{y}\left(X_{y}^{\prime}\right)\right]\right] \\
& =\left(\underset{y \leftarrow}{\mathbb{R}}\left[\operatorname{Pr}\left[A_{y}\left(X_{y}\right)\right]\right]-\underset{y \leftarrow Y^{\prime}}{\mathbb{E}}\left[\operatorname{Pr}\left[A_{y}\left(X_{y}^{\prime}\right)\right]\right]\right) \\
& +\left(\underset{y \leftarrow Y^{\prime}}{\mathbb{R}}\left[\operatorname{Pr}\left[A_{y}\left(X_{y}^{\prime}\right)\right]\right]-\underset{y \leftarrow}{\mathbb{R}}\left[\operatorname{Pr}\left[A_{y}\left(X_{y}^{\prime}\right)\right]\right]\right)
\end{aligned}
$$

Letting $A(x, y)=A_{y}(x)$, we can rewrite the first difference as $\operatorname{Pr}[A(X, Y)]-\left[\operatorname{Pr}\left[A\left(X^{\prime}, Y^{\prime}\right)\right]\right.$ and so it is upper-bounded by $\varepsilon$. The second difference can be written as

$$
\operatorname{Pr}\left[B\left(Y^{\prime}\right)\right]-\operatorname{Pr}[B(Y)]
$$

where $B(y)$ is the randomized function which samples $x \stackrel{R}{\leftarrow} X_{y}^{\prime}$ and outputs $A_{y}(x)$. Since the marginals $Y$ and $Y^{\prime}$ are also $\varepsilon$-close, it follows that the second difference is also upper-bounded by $\varepsilon$, which completes the proof. 
By Fact 7.11 and Eq. 16, a random $\rho \stackrel{R}{\leftarrow} R$ satisfies Eq. (17) with probability $1-\alpha_{1}$. We show that a random $\rho \stackrel{R}{\leftarrow} R$ satisfies Eq. (18) with probability $1-\alpha_{2}$.

Since $\mathbf{R}^{\prime}$ is of bit-length $\ell<s$, we have, by Fact 3.1 ([DORS08, Lemma 2.2b]),

$$
\tilde{\mathbf{H}}_{\infty}\left(\mathbf{T}^{\prime} \mid \mathbf{P}^{\prime}, \mathbf{R}^{\prime}\right)>\tilde{\mathbf{H}}_{\infty}\left(\mathbf{T}^{\prime} \mid \mathbf{P}^{\prime}\right)-s>\log p-\Delta-s,
$$

it follows (by the second part of Fact 3.1) that

$$
\underset{\rho \in R}{\operatorname{Pr}}\left[\tilde{\mathbf{H}}_{\infty}\left(\mathbf{T}_{\mathbf{R}^{\prime}=\rho}^{\prime} \mid \mathbf{P}_{\mathbf{R}^{\prime}=\rho}^{\prime}\right)>\log p-\Delta-s-\log \left(1 / \alpha_{2}\right)\right]>1-\alpha_{2} .
$$

Since $\mathbf{R}^{\prime}$ is $\varepsilon$-close to $R$, we conclude that a random $\rho \stackrel{R}{\leftarrow} R$ satisfies Eq. (18) with probability $1-\alpha_{2}-\varepsilon$. Overall, by a union bound, a random $\rho \stackrel{R}{\leftarrow} R$ is likely to be good with probability $1-\alpha_{1}-\alpha_{2}-\varepsilon$. By letting $\alpha_{1}=1-\varepsilon-3 \alpha / 2$ and $\alpha_{2}=\alpha / 2$, we establish the lemma.

\subsubsection{Removing zerocheck gates}

We move on to take care of zerocheck gates.

Lemma 7.12 (Removing zerocheck gates). Let $p>(s+1) \cdot 2^{s+1}$. For every $\beta>0$, there exists $a \operatorname{poly}(s, \log p, \log (1 / \beta))$-time computable probabilistic mapping $f$ that maps an s-size arithmetic circuit $P: \mathbb{F}^{n} \rightarrow \mathbb{F}^{m}$ over \{zerocheck, add, subtract, multiply, divide, 0,1 \} into an $\left(s+s^{2}\right)$-size arithmetic circuit $f(P): \mathbb{F}^{n+1} \rightarrow \mathbb{F}^{m+1}$ over \{add, subtract, multiply, divide, 0,1$\}$ such that the following hold for every s-size strictly arithmetic circuit $T$ :

- If $(P, T)$ is a Yes instance of $\mathrm{AP}_{d, \varepsilon, p, \Delta}$ then, with probability $1-\beta$, the pair $(f(P), T)$ is a Yes instance of $\mathrm{AP}_{d^{\prime}, \varepsilon^{\prime}, p, \Delta}$.

- If $(P, T)$ is a No instance of $\mathrm{AP}_{d, \varepsilon, p, \Delta}$, then with probability $1-\beta$, the pair $(f(P), T)$ is a No instance of $\mathrm{AP}_{d^{\prime}, \varepsilon^{\prime}, p, \Delta}$.

where $d^{\prime}=d+1$ and $\varepsilon^{\prime}=\varepsilon+\frac{s 2^{s+1}}{p}$.

Note that after the removal of zerocheck gates the mapping $P$ may contain a division by zero. As usual, in this case the output may be arbitrary. We will show that our reduction works regardless of the behavior of the circuit in this case.

Proof. We will use a zerocheck-removal procedure defined in Proposition 3.11. Recall that this procedure outputs, with probability $1-\beta$, a zerocheck-free circuit $\hat{P}: \mathbb{F}^{n} \rightarrow \mathbb{F}^{m}$ of size $s$ together with a strictly arithmetic circuit $G: \mathbb{F}^{n} \rightarrow \mathbb{F}$ of size $s^{2}$ that computes a degree $s 2^{s+1}$ polynomial such that $P$ and $\hat{P}$ agree on all inputs $x$ which are not roots of $G$. Based on $\hat{P}$ and $Z$, we define a new zerocheck-free circuit with $n+1$ inputs and $m+1$ outputs. The first $m$ outputs are computed by $\hat{P}\left(x_{1}, \ldots, x_{n}\right)$. The last $(m+1)$-th output is the polynomial $\hat{G}\left(x_{1}, \ldots, x_{n+1}\right)=G\left(x_{1}, \ldots, x_{n}\right) \cdot x_{n+1}$ where $x_{n+1}$ is a new input variable. (Intuitively, when $x_{n+1}$ is random, $\hat{G}$ contains a single bit of information that signals whether $\hat{P}$ equals to $P$.)

We analyze the effect of the above procedure for Yes instances (assuming that the algorithm from Proposition 3.11 succeeds).

Claim 7.13. If $(P, T)$ is a Yes instance of $\mathrm{AP}_{d, \varepsilon, p, \Delta}$, then $((\hat{P}, \hat{G}), T)$ is a Yes instance of $\mathrm{AP}_{d^{\prime}, \varepsilon^{\prime}, p, \Delta}$. 
Proof. Since $(P, T)$ is a Yes instance there exists a pair of degree $d$ non-zero polynomials $A, B$ : $\mathbb{F}^{m} \rightarrow \mathbb{F}$, such that $\frac{A(P(\boldsymbol{x}))}{B(P(\boldsymbol{x}))}=T(\boldsymbol{x})$ for every $\boldsymbol{x}$ for which $B(P(\boldsymbol{x})) \neq 0$.

Note that $A$ and $B$ are defined over $m$ input variables $z=\left(z_{1}, \ldots, z_{m}\right)$. We extend these polynomials with an additional input variable $z_{m+1}$ via

$$
A^{\prime}\left(z, z_{m+1}\right)=A(z) \cdot z_{m+1}, \quad \text { and } \quad B^{\prime}\left(z, z_{m+1}\right)=B(z) \cdot z_{m+1}
$$

Observe that

$$
\begin{aligned}
\frac{A^{\prime}(\hat{P}(\boldsymbol{x}), \hat{G}(\boldsymbol{x}))}{B^{\prime}(\hat{P}(\boldsymbol{x}), \hat{G}(\boldsymbol{x}))} & =\frac{A(\hat{P}(\boldsymbol{x})) \cdot \hat{G}(\boldsymbol{x})}{B(\hat{P}(\boldsymbol{x}) \cdot \hat{G}(\boldsymbol{x})} \\
& =\frac{A(\hat{P}(\boldsymbol{x}))}{B(\hat{P}(\boldsymbol{x}))} \\
& =\frac{A(P(\boldsymbol{x}))}{B(P(\boldsymbol{x}))}=T(\boldsymbol{x}),
\end{aligned}
$$

where the second equality holds for every $\boldsymbol{x}$ which is not a root of $\hat{G}$, the third equality holds for every $\boldsymbol{x}$ which is not a root of $G$ (since $P$ and $\hat{P}$ agree on all these points), and the last equality holds for every $\boldsymbol{x}$ for which $B(P(\boldsymbol{x})) \neq 0$ (by our assumption). Overall, equality holds for every $\boldsymbol{x}$ which does not zero the denominator $B^{\prime}(\hat{P}(\boldsymbol{x}), \hat{G}(\boldsymbol{x}))$, as required. Finally, $A^{\prime}$ and $B^{\prime}$ are of degree at most $d+1$ and so the claim follows.

We move on to analyze No instances.

Claim 7.14. If $(P, T)$ is a No instance of $\mathrm{AP}_{d, \varepsilon, p, \Delta}$, then $((\hat{P}, \hat{G}), T)$ is a No instance of $\operatorname{AP}_{d^{\prime}, \varepsilon^{\prime}, p, \Delta}$.

Proof. Since $(P, T)$ is a No-instance, the distribution $(P, T)\left(U_{n}\right)$ is $\varepsilon$-close to some distribution $\left(\mathbf{P}^{\prime}, \mathbf{T}^{\prime}\right)$ with $\tilde{\mathbf{H}}_{\infty}\left(\mathbf{T}^{\prime} \mid \mathbf{P}^{\prime}\right)>\log p-\Delta$. Consider the distribution $\left(\left(\mathbf{P}^{\prime}, \mathbf{G}^{\prime}\right), \mathbf{T}^{\prime}\right)$ obtained from $\left(\mathbf{P}^{\prime}, \mathbf{T}^{\prime}\right)$ by concatenating an independent uniform element $\mathbf{G}^{\prime} \stackrel{R}{\leftarrow} \mathbb{F}$. Since $\mathbf{G}^{\prime}$ is independent of $\mathbf{T}^{\prime}$, it still holds that $\tilde{\mathbf{H}}_{\infty}\left(\mathbf{T}^{\prime} \mid \mathbf{P}^{\prime}, \mathbf{G}\right)>\log p-\Delta$ (see Fact 3.1 item 2). We will show that $((\hat{P}, \hat{G}), T)\left(U_{n}\right)$ is $\left(\varepsilon+\frac{s 2^{s+1}}{p}\right)$-close to $\left(\left(\mathbf{P}^{\prime}, \mathbf{G}^{\prime}\right), \mathbf{T}^{\prime}\right)$.

Consider the "hybrid" random variable $\left(\left(P, x_{n+1}\right), T\right)\left(U_{n+1}\right)$ which for $\boldsymbol{x} \stackrel{R}{\leftarrow} \mathbb{F}^{n+1}$ outputs the tuple $\left(P\left(\boldsymbol{x}_{1}, \ldots, \boldsymbol{x}_{n}\right), \boldsymbol{x}_{n+1}, T\left(\boldsymbol{x}_{1}, \ldots, \boldsymbol{x}_{n}\right)\right)$. This random variable is distributed identically to $((\hat{P}, \hat{G}), T)(\boldsymbol{x})$, conditioned on the event $G\left(\boldsymbol{x}_{1}, \ldots, \boldsymbol{x}_{n}\right) \neq 0$. By Schwartz-Zippel, this event happens with probability at most $\frac{s 2^{s+1}}{p}$, and therefore the two distributions are $\frac{s 2^{s+1}}{p}$-close.

In addition, it is not hard to verify that $\left(\left(P, x_{n+1}\right), T\right)\left(U_{n+1}\right)$ is $\varepsilon$-close to the distribution $\left(\left(\mathbf{P}^{\prime}, \mathbf{G}^{\prime}\right), \mathbf{T}^{\prime}\right)$. (Indeed, $\left(\left(P, x_{n+1}\right), T\right)\left(U_{n+1}\right)$ is obtained from $(P, T)\left(U_{n}\right)$ via the same randomized mapping that derives $\left(\left(\mathbf{P}^{\prime}, \mathbf{G}^{\prime}\right), \mathbf{T}^{\prime}\right)$ from $\left(\mathbf{P}^{\prime}, \mathbf{T}^{\prime}\right)$.) It follows, by the transitivity of statistical distance, that $((\hat{P}, \hat{G}), T)\left(U_{n}\right)$ is $\varepsilon+\frac{s 2^{s+1}}{p}$-close to $\left(\left(\mathbf{P}^{\prime}, \mathbf{G}^{\prime}\right), \mathbf{T}^{\prime}\right)$, and the claim follows.

This completes the proof of Lemma 7.12 


\subsubsection{Removing Division Gates}

Lemma 7.15 (Removing divide gates). There exists an efficiently computable (deterministic) mapping $f$ that maps an s-size arithmetic circuit $P: \mathbb{F}^{n} \rightarrow \mathbb{F}^{m}$ over add, subtract, multiply, divide, 0,1$\}$ into an $4 s$-size arithmetic circuit $f(P): \mathbb{F}^{n+m} \rightarrow \mathbb{F}^{2 m}$ over add, subtract, multiply, 0,1$\}$ such that the following hold for every s-size strictly arithmetic circuit $T$ :

- If $(P, T)$ is a Yes instance of $\mathrm{AP}_{d, \varepsilon, p, \Delta}$ then the pair $(f(P), T)$ is a Yes instance of $\mathrm{AP}_{d^{\prime}, \varepsilon, p, \Delta}$.

- If $(P, T)$ is a No instance of $\mathrm{AP}_{d, \varepsilon, p, \Delta}$ then the pair $(f(P), T)$ is a No instance of $\mathrm{AP}_{d^{\prime}, \varepsilon, p, \Delta}$.

where $d^{\prime}=2 d+m$.

Proof. We assume that $P_{i}$, the $i$-th output of $P$, is described by a circuit of the form $A_{i} / B_{i}$ where $A_{i}$ and $B_{i}$ are arithmetic circuits of size $s^{\prime} \leq 4 s$ over $\{$ add, subtract, multiply, 0,1$\}$ that compute polynomials of degree at most $2^{s}$. This is without loss of generality, since any arithmetic circuit $P_{i}$ over $\{$ add, subtract, multiply, divide, 0,1$\}$ can be transformed into this form at the expense of increasing its size by a factor of 4 , cf. [SY10, Proof of Thm. 2.11]. We define a new instance to the problem by keeping the same target polynomial $T$ and modifying $P$ to $f(P)=\left(A_{i}^{\prime}, B_{i}^{\prime}\right)_{i=1}^{m}$ where

$$
A_{i}^{\prime}\left(x_{1}, \ldots, x_{n}, r_{i}\right)=A_{i}(x) \cdot r_{i}, \quad B_{i}^{\prime}\left(x_{1}, \ldots, x_{n}, r_{i}\right)=B_{i}(x) \cdot r_{i}
$$

and $r=\left(r_{1}, \ldots, r_{m}\right)$ are new input variables. (Formally, we redefine $T$ as $\left.T(x, r)=T(x)\right)$.

We claim that if the original instance was a Yes instance then the new instance is also a Yes instance. Assume that there exists $Q=A / B$ such that $Q(P(\boldsymbol{x}))=T(\boldsymbol{x})$ for all $\boldsymbol{x} \in \mathbb{F}^{n}$ which are not roots of $B$. Define the rational function $Q^{\prime}\left(A_{1}^{\prime}, B_{1}^{\prime}, \ldots, A_{m}^{\prime}, B_{m}^{\prime}\right)$ which outputs $Q\left(A_{1}^{\prime} / B_{1}^{\prime}, \ldots, A_{m}^{\prime} / B_{m}^{\prime}\right) \cdot\left(\prod_{i} B_{i}^{\prime}\right) /\left(\prod_{i} B_{i}^{\prime}\right)$. For every $\boldsymbol{x}, \boldsymbol{r}$ which are not roots of $\prod_{i} B_{i}^{\prime}$, we have that $Q^{\prime}(f(P)(\boldsymbol{x}, \boldsymbol{r}))=Q(P(\boldsymbol{x}))$. Observe that $\boldsymbol{x}, \boldsymbol{r}$ which are roots of $\prod_{i} B_{i}^{\prime}$ are, by construction, roots of the denominator of $Q^{\prime}$. It follows that $(f(P), T)$ is indeed a Yes instance of $\mathrm{AP}_{d^{\prime}, \varepsilon^{\prime}, p, \Delta^{\prime}}$ where the degree $d^{\prime}$ of the nominator and denominator of $Q$ (viewed as polynomials in $2 m$ variables) are at most $d^{\prime}=2 d+m$.

We move on to analyze the case of a No instance. Assume that the distribution $(P, T)\left(U_{n}\right)$ is $\varepsilon$ close to some distribution $\left(\mathbf{P}^{\prime}, \mathbf{T}^{\prime}\right)$ with $\tilde{\mathbf{H}}_{\infty}\left(\mathbf{T}^{\prime} \mid \mathbf{P}^{\prime}\right)>\log p-\Delta$. Consider the randomized mapping $g$ which given a vector $p \in \mathbb{F}^{m}$ samples $r \stackrel{R}{\leftarrow} \mathbb{F}^{m}$ and outputs the vector $\left(p_{1} r_{1}, r_{1}, \ldots, p_{m} r_{m}, r_{m}\right) \in$ $\mathbb{F}^{2 m}$. It is not hard to verify that the distribution $\left(g\left(P\left(U_{n}\right)\right), T\left(U_{n}\right)\right)$ is distributed identically to $\left(f(P)\left(U_{n}\right), T\left(U_{n}\right)\right)$. It follows that $\left(f(P)\left(U_{n}\right), T\left(U_{n}\right)\right)$ is $\varepsilon$-close to $\left(g\left(\mathbf{P}^{\prime}\right), \mathbf{T}^{\prime}\right)$. Finally, by Fact 3.1 (item 2), $\tilde{\mathbf{H}}_{\infty}\left(\mathbf{T}^{\prime} \mid g\left(\mathbf{P}^{\prime}\right)\right)>\tilde{\mathbf{H}}_{\infty}\left(\mathbf{T}^{\prime} \mid \mathbf{P}^{\prime}\right)>\log p-\Delta$. Hence, $(f(P), T)$ is a No instance of $\mathrm{AP}_{d^{\prime}, \varepsilon, p, \Delta}$. The Lemma follows.

\subsubsection{Putting It All Together (Proving Theorem 7.2, Part 2)}

We employ Lemmas 7.10, 7.12 and 7.15 to reduce an instance $(P, T)$ of $\operatorname{AP}_{d, \varepsilon, p, \Delta}$ to a strictly arithmetic instance of $\mathrm{AP}_{d^{\prime}, \varepsilon^{\prime}, \Delta^{\prime}, p}$, and then apply the first part of Theorem 7.2, Details follow.

Given an $s$-size arithmetic circuit $P: \mathbb{F}^{n} \rightarrow \mathbb{F}^{m}$ and an $s$-size strictly arithmetic circuit $T$ : $\mathbb{F}^{n} \rightarrow \mathbb{F}$, we transform $P$ into a strictly arithmetic circuit $P^{\prime}$ as follows: (1) change the sample gates to be input gates; (2) remove the bitsample gates by applying Lemma 7.10 with $\alpha=\frac{2}{3}\left(\frac{1}{5}-\varepsilon\right)$; (3) remove the zerocheck gates by applying Lemma 7.12 with $\beta=2^{-s}$; and (4) remove the divide gates 
by applying Lemma 7.15. The resulting circuit $P^{\prime}$ has $n^{\prime}=n+m+2 \leq 2 s$ inputs, $m^{\prime}=2 m+2 \leq 2 s$ outputs, and size $s^{\prime}=4\left(s+s^{2}\right) \leq 5 s^{2}$. Let

$$
d^{\prime}=2 d+m+3, \quad \varepsilon^{\prime}=\frac{2 \varepsilon}{1-\varepsilon-3 \alpha / 2}+\frac{s 2^{s+1}}{p}, \quad \Delta^{\prime}=\Delta+s+1+\log (1 / \alpha) .
$$

If $(P, T)$ is a Yes instance then, with probability $1-\beta$, the pair $\left(P^{\prime}, T\right)$ is a Yes instance of $\operatorname{AP}_{d^{\prime}, \varepsilon^{\prime}, \Delta^{\prime}, p}$. If $(P, T)$ is a No instance then, with probability $\alpha-\beta$, the pair $\left(P^{\prime}, T\right)$ is a Yes instance of $\mathrm{AP}_{d^{\prime}, \varepsilon^{\prime}, \Delta^{\prime}, p}$.

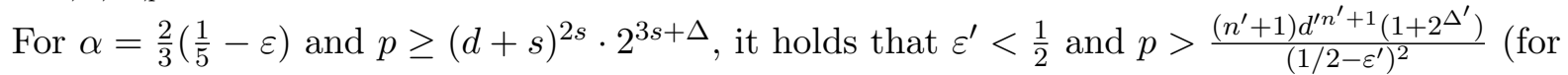
all sufficiently large $s$ ). Therefore, the first part of Theorem 7.2 guarantees an algorithm $\mathcal{A}$ that decides whether $\left(P^{\prime}, T\right)$ is Yes instance or a No instance of $A \mathrm{P}_{d^{\prime}, \varepsilon^{\prime}, \Delta^{\prime}, p}$ with an error of $\beta$ in time poly $\left(s^{\prime}, \log (1 / \beta), \log (p)\right)$. (The low error can be obtained via standard amplification techniques.) We output the value $\mathcal{A}\left(P^{\prime}, T\right)$.

Overall, if $(P, T)$ is a Yes instance then we answer correctly with probability $1-2 \beta$, and if $(P, T)$ is a No instance, we answer correctly with probability $\alpha^{\prime}=\alpha-2 \beta$. We repeat the above procedure $t=\ln (1 / \beta) / \alpha^{\prime}=\operatorname{poly}(s)$ times (with independent randomness) and output "No" if at least one of the iterations outputs "No". We will err on a No instance with probability $\left(1-\alpha^{\prime}\right)^{t}<\beta=2^{-s}$ and err on a Yes instance with probability $3 t \beta=2^{-\Omega(s)}$. This completes the proof of the second part of Theorem 7.2 .

\section{Part II}

\section{Positive Results}

In the following sections we give constructions of cryptographic primitives in the arithmetic model. We start by introducing the RLC assumption on which we base our constructions (Section 8), and then provide constructions for arithmetic pseudorandom generator (Section 9), symmetric and public key encryption (Section 10), commitments (Section 11) and protocols for secure computation (Section 12).

As explained in the introduction, we present several alternative constructions based on three different approaches: (1) an abstract primitive (Arithmetic/Binary one-time encryption) that allows to import binary constructions to the arithmetic setting; (2) direct approach which adopt known LPN-based construction to the arithmetic setting; and (3) a reduction-based approach that exploits the fact that some classical cryptographic transformations have a simple arithmetic variant.

Remark 7.16 (Adversarial model). From now on, we move to a non-uniform model and use the term efficient adversary to denote a probabilistic polynomial-time (binary) circuit family. This choice is taken to simplify the presentation, and all our results can be easily adopted to the uniform model.

\section{The RLC Assumption}

We present the RLC assumption of [IPS09] which asserts that a noisy codeword of a random linear code is pseudorandom. 
Notation. For a field $\mathbb{F}$, integer $\ell$ and real $p \in(0,1)$, let $\chi_{p}^{\ell}(\mathbb{F})$ denote the probability distribution over $\mathbb{F}^{\ell}$ where each coordinate takes the value zero with probability $1-p$ and a random element in $\mathbb{F}$ with probability $p$. When the field is clear from the context, we omit it, and write $\chi_{p}^{\ell}$.

Assumption $8.1(\operatorname{RLC}(n, \ell, p))$. For security parameter $n$, length parameter $\ell(n)$, noise parameter $p(n) \in(0,1)$ the $\operatorname{RLC}(n, \ell, p)$ assumption asserts that for every efficient adversary $\mathcal{A}$ the probability of winning the following game is at most $\frac{1}{2}+\operatorname{neg}(n)$ :

IND-Game $\left(1^{n}\right)$ :

- $\mathcal{A}$ receives $1^{n}$, chooses a field's implementation $\mathbb{F}$ and sends $\mathbb{F}$ to the challenger.

- The challenger samples a challenge bit $b \stackrel{R}{\leftarrow}\{0,1\}$ and sends $\left(M, c_{b}\right)$ to $\mathcal{A}$ where $M \stackrel{R}{\leftarrow}$ $\mathbb{F}^{\ell(n) \times n}, c_{0} \stackrel{R}{\leftarrow} \mathbb{F}^{\ell(n)}$ and $c_{1}=M \cdot s+e$ where $s \stackrel{R}{\leftarrow} \mathbb{F}^{n}, e \stackrel{R}{\leftarrow} \chi_{p(n)}^{\ell(n)}$.

- $\mathcal{A}$ outputs $b^{\prime}$ and wins if $b=b^{\prime}$.

\section{Remarks:}

- Observe that the $\operatorname{RLC}(n, \ell, p)$ assumption is equivalent to the assumption that for every efficient field family $\left\{\mathbb{F}_{n}\right\}$ it holds that $\mathcal{D}_{\mathbb{F}, p}^{\ell \times n} \stackrel{c}{\approx}\left(M \stackrel{R}{\leftarrow} \mathbb{F}_{n}^{\ell \times n}, v \stackrel{R}{\leftarrow} \mathbb{F}_{n}^{\ell}\right)$.

- It is not hard to see that RLC can only become harder when the noise $p$ increases and the length $\ell$ decreases. Hence, it is desirable to use the assumption with high noise and short matrices.

- The error distribution $\chi_{p}^{\ell}$ can be efficiently sampled up to negligible statistical distance by an arithmetic circuit that receives as input $\left(H_{2}(p)+\varepsilon\right) \cdot \ell$ random bits and $(p+\varepsilon) \cdot \ell$ random field elements. The circuit first assigns for each output element the binary outcome of whether it carries noise at all or not. For $p$ values of noticeable magnitude, for each output this can be sampled by a binary circuit that uses $H_{2}(p)+\varepsilon$ random input bits, where $H_{2}(p)$ denotes the binary entropy of $p$. This binary circuit can be simulated by an arithmetic circuit of the same size that takes as input only random bits. Once the noisy coordinates are decided a random noise from $\mathbb{F}$ is assigned to each of them, with overwhelming probability there will be less than $(p+\varepsilon) \cdot \ell$ noisy coordinates.

\section{Arithmetic Pseudorandom Generator}

In this section, we construct an arithmetic PRG (APRG) with arbitrary polynomial stretch based on the RLC assumption.

\subsection{Basic Observations}

Recall that APRG $=\left\{\mathrm{APRG}_{n}\right\}$ is viewed as a randomized circuit which samples a pseudorandom distribution over $\mathbb{F}^{\ell(n)}$ using $n$ randomized gates. Following our general convention, we allow the PRG to use both sample gates and bitsample gates. (This is also motivated by our applications that can tolerate the use of such gates.) In the following we observe that the number of bitsample gates can be always reduced via the use of a binary PRG. 
Observation 9.1. Assume that $A P R G=\left\{\mathrm{APRG}_{n}\right\}$ outputs $\ell(n)$ pseudorandom field elements (as per the second item of the above definition) and uses $n<\ell(n)$ gates that sample random field elements (sample), and $k(n)$ gates that sample random bits (bitsample), where $k(n)$ may be larger than $\ell(n)$. Then, for every constant $\varepsilon>0$, there exists an arithmetic PRG $\mathrm{APRG}^{\prime}=\left\{\mathrm{APRG}_{n}^{\prime}\right\}$ that samples $\ell(n)$ pseudorandom field elements and uses $n$ sample gates and $n^{\varepsilon}$ bitsample gates. Furthermore, if APRG is simple (i.e., does not contain division or zerocheck gates) then so is APRG'.

Proof. We use a binary pseudorandom generator (PRG) which stretches $n^{\varepsilon}$ bits to $k(n)$ pseudorandom bits. The new APRG samples $n^{\varepsilon}$ bits, feeds them to the PRG, and uses the $k(n)$ outputs instead of the original bitsample gates. It is not hard to show that the outcome is pseudorandom (otherwise one can break the PRG). Furthermore, the PRG can be written in arithmetic form by arithmetizing the boolean logic (i.e., replace AND with multiplication and $\operatorname{NOT}(w)$ with $1-w$ ). Finally, the existence of a binary PRG follows from the hypothesis of the theorem. Indeed, a binary PRG is directly obtained by instantiating APRG with a sufficiently large field $|\mathbb{F}|>2^{2 \ell}$ and by using a proper implementation of the field (i.e., that guarantees that a random field elements is represented by a uniform, or close-to-uniform, string).

We do not know whether it is possible to completely eliminate bitsample gates. The existence of an arithmetic PRG that does not use random bits at all is left as an interesting open problem.

Length Expansion. We continue by showing that given a minimal APRG which expands $n$ inputs to $n+1$ outputs, one can construct an APRG' with polynomial expansion $n^{c}$. The transformation is similar to the standard transformation from the binary setting [Gol01, Chapter 3.3].

Lemma 9.2 (APRG Expansion). Suppose that there exists an Arithmetic PRG APRG with an additive expansion of 1 . Then, for any polynomial $q(n)=\operatorname{poly}(n)$, there exists an Arithmetic PRG APRG' with an output length of $q(n)$ where $n$ denotes the seed length. Furthermore, if APRG is simple, then so is APRG'.

Sketch. In the following, we assume, WLOG, that the underlying APRG $G$ uses $n_{1}$ sample gates and $n_{2}$ bitsample gates, where $n_{1}+n_{2}=n$. Furthermore, we view the random gates of the APRG as input gates, and accordingly view the APRG as a function $G: \mathbb{F}^{n_{1}} \times\{0,1\}^{n_{2}} \rightarrow \mathbb{F}^{n+1}$. We will construct a new function $\mathrm{APRG}^{\prime}: \mathbb{F}^{n_{1}} \times\{0,1\}^{q \cdot n_{2}} \rightarrow \mathbb{F}^{n+1}$ whose output is pseudorandom when evaluated on a random input. Then, we use Observation 9.1 to reduce the number of binary inputs to $n_{2}$. The function APRG' is defined iteratively.

- Given a seed $a_{0} \in \mathbb{F}^{n_{1}}$ and $b=\left(b_{0}, \ldots, b_{q-1}\right)$ where $b_{i} \in\{0,1\}^{n_{2}}$.

- For $i=0$ to $q-1$ : Compute $G\left(a_{i}, b_{i}\right) \in \mathbb{F}^{n+1}$ and parse the result as $\left(a_{i+1}, y_{i+1}\right) \in \mathbb{F}^{n_{1}} \times$ $\mathbb{F}^{(n+1)-n_{1}}$.

- Output $y_{1}, \ldots, y_{q}$.

The proof of security follows from a standard hybrid argument. For $j=0, \ldots, q$ define the hybrid $H_{j}$ in which for every $i \leq j$ the values $\left(a_{i}, y_{i}\right) \stackrel{R}{\leftarrow} \mathbb{F}^{n_{1}} \times \mathbb{F}^{(n+1)-n_{1}}$ are uniformly chosen, and the other iterations remain unchanged. The hybrid $H_{0}$ corresponds to the real construction, while the outcome of the last hybrid $H_{q}$ is clearly uniform. We show that $H_{0}$ is indistinguishable from 
$H_{q}$, by showing that a distinguisher $\mathcal{A}$ that $\varepsilon$-distinguishes these distributions can be used to violate the pseudorandomness of $G$. Given a challenge $z \in \mathbb{F}^{n+1}$ we sample a random location $j \in\{0, \ldots, q-1\}$, sample the first $j-1$ values $\left(a_{i}, y_{i}\right)_{i<j}$ uniformly, let $\left(a_{j}, b_{j}\right)=z$, and continue for the other iterations as in the real constructions. The output is given to the distinguisher $\mathcal{A}$. It is not hard to show that the distinguishing advantage of the new distinguisher is $\varepsilon / q$, and so the lemma follows.

\subsection{Construction based on RLC}

We continue with an arithmetic construction of an APRG based on the RLC assumption. Recall that the $\operatorname{RLC}(n, \ell, p)$ assumption asserts that for a random matrix $M \stackrel{R}{\leftarrow} \mathbb{F}^{\ell \times n}$, a random vector

$s \stackrel{R}{\leftarrow} \mathbb{F}^{n}$ and an error vector $e \stackrel{R}{\leftarrow} \chi_{p}^{\ell}(\mathbb{F})$, the output $(M, M s+e)$ is pseudorandom. This gives an immediate construction of an APRG.

Theorem 9.3. Assuming $\operatorname{RLC}\left(n, \ell=\frac{n}{1-p-\varepsilon}, p\right)$ holds for some constants $p \in(0,1), \varepsilon>0$, there exists a simple APRG with an arbitrary polynomial output length.

Proof. The circuit samples a random matrix $M \stackrel{R}{\leftarrow} \mathbb{F}^{\ell \times n}$, a random vector $s \stackrel{R}{\leftarrow} \mathbb{F}^{n}$, and computes $y=M s$. The noise is sampled as described in Section 8 . First, we use a polynomial number of bitsample gates to sample a binary vector of $\ell$ independent Bernoulli random variables $\alpha=$ $\left(\alpha_{1}, \ldots, \alpha_{\ell}\right)$ each with a probability of success $p$. If the number of ones, $t$, is smaller than $(p+\varepsilon) \ell$, we sample $t$ random field elements and place them in the 1's locations of $\alpha$, the resulting noise vector $e$ is then added to $y$ and the output is $(M, M s+e)$. If $t \geq(p+\varepsilon) \ell$, we let $e$ be the all-zero vector. By Chernoff bound, the latter event happens with probability $2^{-\Omega(\ell)}$, and so the resulting distribution is statistically close to the RLC distribution, which is pseudorandom by assumption. It follows that the output of the generator is also pseudorandom. Since the number of random field elements in the seed is strictly smaller than $\ell n+n+(p+\varepsilon) \ell$ which is upper-bounded by the output length $\ell n+\ell$, we can apply Observation 9.1 and Lemma 9.2, and get an APRG with an arbitrary polynomial output length.

\section{Encryption}

In this section, we construct arithmetic encryption schemes in the public-key and symmetric-key setting based on an RLC assumption. We begin with a construction of a one-time secure symmetric encryption scheme which encrypts field elements using binary keys. We use this arithmetic/binary encryption (ABE) scheme to obtain CPA-secure arithmetic encryption schemes in the symmetrickey and public-key settings (Section 10.2). Finally, we show that known LPN-based constructions arithmetize and yield arithmetic symmetric encryption schemes (Section 10.3) and public-key encryption schemes (Section 10.4).

\subsection{One-Time Secure Arithmetic/Binary Encryption}

Recall that arithmetic/binary encryption (ABE) is an arithmetic symmetric encryption scheme with one-time security with the additional property that all the elements of the secret key sk are taken from the subset $\{0,1\} \subset \mathbb{F}$. That is, the key is essentially a string of bits given as a sequence of 0-1 Field elements. (See Definition 4.2.) As we will see this special property can be used as a 
bridge from the binary model to the arithmetic model. We begin with a simple construction of $\mathrm{ABE}$ based on the RLC assumption.

Notation. For an integer $\ell$ and real $p \in(0,1)$, let $\mathcal{B}_{p}^{\ell}$ denote the probability distribution over $\{0,1\}^{\ell}$ where each coordinate takes the value one with probability $p$.

Construction 10.1 (Arithmetic/Binary Encryption). We parameterize our construction by a constant $p \in(0,1)$ and a constant $\varepsilon>0$. For a security parameter $n$, we set the length parameter $\ell:=\frac{(1+\varepsilon) n}{1-p}$. The scheme consists of the following circuits:

- $\mathrm{KGen}_{n}$ : Sample $e \stackrel{R}{\leftarrow} \mathcal{B}_{p}^{\ell}$.

- $\operatorname{Enc}_{n}^{\mathbb{F}}(e, x)$ : Sample $A \stackrel{R}{\leftarrow} \mathbb{F}^{\ell \times n}, s \stackrel{R}{\leftarrow} \mathbb{F}^{n}$, and $u \stackrel{R}{\leftarrow} \mathbb{F}^{\ell}$. Set diagonal matrix $U$ s.t. $U_{i i}=u_{i}$. Output $\left(A, c:=A s+U \cdot e+x^{\ell}\right)$, where $x^{\ell}$ denotes the $\ell$ long (column ) vector $(x, \ldots, x)$.

- $\operatorname{Dec}_{n}^{\mathbb{F}}(e,(A, c))$ : Find an assignment to the variables $y, z=\left(z_{1}, \ldots, z_{n}\right)$ which satisfies the linear equation system $A^{\prime} z+y^{\ell^{\prime}}=c^{\prime}$ where $A^{\prime}$ and $c^{\prime}$ contain the $\ell^{\prime}$ non-noisy coordinates of $e$, and output the value that is assigned to $y$.

We notice that the decryption can be implemented arithmetically by solving the system of linear equations $(I-E) c=(I-E)\left(A z+y^{\ell}\right)$, where $E$ is the diagonal matrix with $E_{i i}=e_{i}$.

Before establishing correctness we will need the following simple lemma.

Lemma 10.2. The following holds for any finite field $\mathbb{F}$, constant $p \in(0,1)$, constant $\varepsilon>0$, and $\ell \geq \frac{(1+\varepsilon) n}{1-p}$. For any vector $c \in \mathbb{F}^{\ell}$, with overwhelming probability over the choice of $A \stackrel{R}{\leftarrow} \mathbb{F}^{\ell \times n}$ and $e \stackrel{R}{\leftarrow} \chi_{p}^{\ell}$, the following linear system over the formal variables $z=\left(z_{1}, \ldots, z_{n}\right)$ and $y$ has no more than a single solution:

$$
B \cdot c=B \cdot\left(A \cdot z+y^{\ell}\right)
$$

where $B \in \mathbb{F}^{\ell \times \ell}$ is defined as the diagonal matrix in which $B_{i i}=1 \Longleftrightarrow e_{i}=0$.

Proof. Let $c^{\prime}=B c$ and $A^{\prime}=B A$ and let $\ell^{\prime}$ be the number of non-noisy coordinates in $e$. Then, it suffices to show that the linear system $c^{\prime}=A^{\prime} z+y^{\ell^{\prime}}$ has a single solution. Rearrange the equation as

$$
\left(\begin{array}{ll}
A^{\prime} & 1^{\ell^{\prime}}
\end{array}\right)\left(\begin{array}{l}
z \\
y
\end{array}\right)=c^{\prime}
$$

Subtract the last equation from all other equations, and denote the resulting matrix (with the last row omitted) by $\left(A^{\prime \prime} \mid 0^{\ell^{\prime \prime}}\right)$ where $A^{\prime \prime} \in \mathbb{F}^{\left(\ell^{\prime \prime}\right) \times n}$ and $\ell^{\prime \prime}=\ell^{\prime}-1$. Observe that if $A^{\prime \prime}$ has full rank, then $z$ is uniquely determined, and (by using the omitted last row) $y$ is also uniquely determined. Hence, it suffices to show that with all but negligible probability $A^{\prime \prime}$ has full rank.

Let us first condition on the event that $\ell^{\prime} \geq(1+\varepsilon / 2) n$ which, by a Chernoff-bound, happens with all but negligible probability. Next, recall that $A^{\prime} \stackrel{R}{\leftarrow} \mathbb{F}^{\ell^{\prime} \times n}$, and so the matrix $A^{\prime \prime} \in \mathbb{F}^{\ell^{\prime \prime} \times n}$ is also uniformly distributed. In this case, the probability that $A^{\prime}$ does not have full rank is less than $|\mathbb{F}|^{-\left(\ell^{\prime \prime}-1\right)+n}=\operatorname{neg}(n)$ (cf. [Fel07]). The lemma now follows by a union-bound.

The following two lemmas establish the correctness and the security of the scheme. 
Lemma 10.3 (correctness). For every field $\mathbb{F}$, and for all $x \in \mathbb{F}$ it holds that

$$
\underset{e{ }_{e}^{R} \mathrm{RGen}_{n}}{\operatorname{Pr}}\left[\operatorname{Dec}_{n}^{\mathbb{F}}\left(e, \operatorname{Enc}_{n}^{\mathbb{F}}(e, x)\right)=x\right] \geq 1-\operatorname{neg}(n)
$$

Proof. Consider the set of linear equations solved at decryption.

$$
c^{\prime}=A^{\prime} z+y^{\ell^{\prime}}
$$

Let us expand the left hand side of the above equation using the definition of $c^{\prime}$, the "clean" coordinates in $c$ :

$$
c^{\prime}=A^{\prime} s+x^{\ell^{\prime}}
$$

clearly $z=s$ and $y=x$ is a valid solution for the above equation set. Lemma 10.2 demonstrates that with overwhelming probability there is no no more than one solution, hence the correctness holds.

Lemma 10.4 (Security). Let $p \in(0,1)$ and $\varepsilon>0$ be some constants. Suppose that the $\operatorname{RLC}(n, \ell=$ $\left.\frac{(1+\varepsilon) n}{1-p}, p\right)$ assumption holds, then Construction 10.1 (instantiated with the same $p$ and $\varepsilon$ ) is one-time computationally secure.

Proof. Assume towards a contradiction that an efficient adversary $\mathcal{A}$ wins the One-Time IND game with probability $1 / 2+\delta(n)$ for some non-negligible function $\delta$. We define a new adversary $\mathcal{A}^{\prime}$ against the RLC assumption in the following way:

\section{Adversary $\mathcal{A}^{\prime}$ :}

- Initialize $\mathcal{A}$ and get a field $\mathbb{F}$ from it. Send the same $\mathbb{F}$ to the challenger.

- Receive from the challenger $(A, v) \in\left(\mathbb{F}^{\ell \times n}, \mathbb{F}^{\ell}\right)$

- Receive from $\mathcal{A}$ a pair $x_{0}, x_{1} \in \mathbb{F}$.

- Sample $b \in\{0,1\}$, and send to $\mathcal{A}$ the challenge $C=\left(A, v+x_{i}^{\ell}\right)$.

- If $\mathcal{A}$ wins, return 0 , else return 1 .

If $\mathcal{A}^{\prime}$ receives $(A, v) \stackrel{R}{\leftarrow} \mathcal{D}_{\mathbb{F}, p}^{\ell \times n}$, then $C$ is distributed as a random cyphertext and so by assumption $\mathcal{A}$ wins with the probability $\frac{1}{2}+\delta(n)$, and $\mathcal{A}^{\prime}$ outputs 0 . However if $(A, v) \stackrel{R}{\leftarrow}\left(\mathbb{F}^{\ell \times n}, \mathbb{F}^{\ell}\right)$, then by the security of the one time pad $\mathcal{A}$ outputs 0 with probability exactly $1 / 2$. And so we get that $\mathcal{A}^{\prime}$ distinguishes $\mathcal{D}_{\mathbb{F}, p}^{\ell \times n}$ from $\left(\mathbb{F}^{\ell \times n}, \mathbb{F}^{\ell}\right)$ with the non-negligible advantage $\frac{\delta(n)}{2}$.

Corollary 10.5. If, for some constants $p \in(0,1), \varepsilon>0$, the assumption $\operatorname{RLC}\left(n, \ell=\frac{(1+\varepsilon) n}{1-p}, p\right)$ holds, then there exists an ABE scheme.

Remark 10.6 (Non-trivialty). The Learning Parity with Noise assumption is trivially hard (in an information-theoretic sense) when the noise rate is $\frac{1}{2}$. In contrast, the $\operatorname{RLC}(n, \ell, p)$ assumption is non-trivial (i.e., implies the existence of a one-way function) for any constant value of $p \in(0,1)$ as it should hold over fields of size larger than $1 / p$. Indeed, when $\mathbb{F}$ is sufficiently large, the entropy needed to sample an $\operatorname{RLC}(n, \ell, p)$ instance (roughly $\log |\mathbb{F}| \cdot(\ell n+n+p \ell)+\ell \cdot H_{2}(p)$ ) is smaller than the entropy of the output $(\ell \log |\mathbb{F}|)$ and so the assumption immediately implies the existence of a pseudorandom generator. 
Remark 10.7 (Weak homomorphism). Interestingly, Construction 10.1 is (weakly) homomorphic under addition and multiplication. Given a ciphertext $(A, c) \stackrel{R}{\leftarrow} \operatorname{Enc}_{n}^{\mathbb{F}}(e, x)$, one can create, for every message $y \in \mathbb{F}$ and every scalar $a \in \mathbb{F}$, a ciphertext $\left(A, c^{\prime}\right) \stackrel{R}{\leftarrow} \operatorname{Enc}_{n}^{\mathbb{F}}(e, a \cdot x+y)$ by computing $c^{\prime}=a \cdot c+y^{\ell}$. This homomorphism is weak in the sense that the joint distribution $(A, c),\left(A, c^{\prime}\right)$ is statistically far from a fresh pair of ciphertexts $\left(\operatorname{Enc}_{n}^{\mathbb{F}}(e, x)\right.$, $\operatorname{Enc}_{n}^{\mathbb{F}}(e, a \cdot x+y)$. For this reason, our impossibility result (Section 7 ) do not apply here.

\subsection{From ABE to Symmetric and Public-Key Encryption}

Next, we show how to employ ABE (which offers only one-time security) in order to obtain CPAsecure arithmetic encryption scheme either in the public or in the symmetric setting. The idea is to combine $\mathrm{ABE}$ and standard binary encryption via a hybrid mode.

Construction 10.8. Let (BGen, BEnc, BDec) be any binary CPA-secure encryption scheme (either in the symmetric setting or in the public-key setting) and let (ABGen, ABEnc, ABDec) be any ABE. Consider the following Arithmetic Encryption Scheme:

- $\mathrm{KGen}_{n}$ : output (sk, pk) $\stackrel{R}{\leftarrow} \mathrm{BGen}_{n}$.

- $\operatorname{Enc}_{n}^{\mathbb{F}}(\mathrm{pk}, x)$ : sample $e \stackrel{R}{\leftarrow} \mathrm{ABGen}_{n}$ output $\left(c_{1} \stackrel{R}{\leftarrow} \mathrm{BEnc}_{n}(\mathrm{pk}, e), c_{2} \stackrel{R}{\leftarrow} \mathrm{ABEnc}_{n}^{\mathbb{F}}(e, x)\right)$.

- $\operatorname{Dec}_{n}^{\mathbb{F}}\left(\mathrm{sk}, c_{1}, c_{2}\right)$ : Compute $e^{\prime}=\operatorname{BDec}_{n}\left(\mathrm{sk}, c_{1}\right)$ and output $x^{\prime}=\operatorname{ABDec}_{n}^{\mathbb{F}}\left(e^{\prime}, c_{2}\right)$.

Correctness follows directly from the correctness of the underlying binary encryption scheme and $\mathrm{ABE}$ scheme.

Lemma 10.9 (Security). Suppose that (BGen, BEnc, BDec) is a CPA-secure public-key encryption scheme (resp., symmetric encryption scheme) and (ABGen, ABEnc, ABDec) is a one-time secure $A B E$, then Construction 10.8 is CPA-secure public-key (resp., symmetric key) arithmetic encryption scheme.

Proof. Let $\mathcal{A}$ be an adversary that attempts to win the standard IND-CPA game. To analyze $\mathcal{A}$ 's success probability, we define (in Table 2) several variants of the original game.

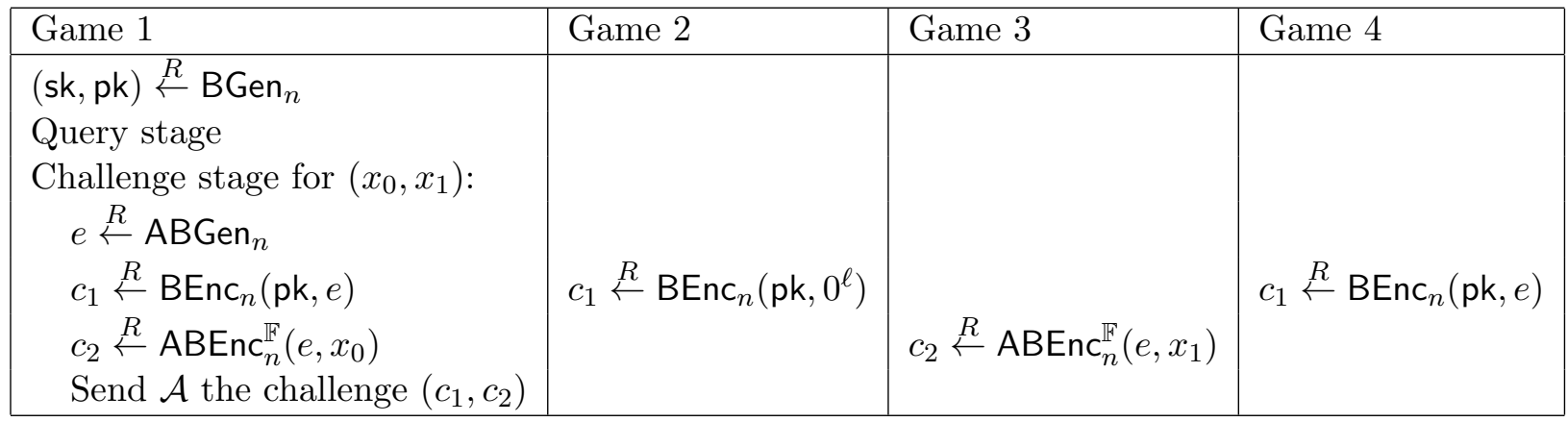

Table 2: Hybris games. In each game only the changes from the previous game are written. In the public-key setting the key pk is given to the adversary at the beginning of the query stage, and in the private-key setting, we let $\mathrm{pk}=\mathrm{sk}$ and keep it hidden from the adversary. 
Let $\operatorname{Pr}\left[\left\langle\mathcal{A}, G_{i}\right\rangle=1\right]$ be the probability that $\mathcal{A}$ returns 1 when interacting with the $i$ 'th game, then the following holds:

1. $\left|\operatorname{Pr}\left[\left\langle\mathcal{A}, G_{1}\right\rangle=1\right]-\operatorname{Pr}\left[\left\langle\mathcal{A}, G_{2}\right\rangle=1\right]\right|=\operatorname{neg}(n)$, else one could break the CPA security of the binary encryption scheme on the challenge $\left(e, 0^{\ell}\right)$.

2. $\left|\operatorname{Pr}\left[\left\langle\mathcal{A}, G_{2}\right\rangle=1\right]-\operatorname{Pr}\left[\left\langle\mathcal{A}, G_{3}\right\rangle=1\right]\right|=\operatorname{neg}(n)$, else one could break the one-time security of the Arithmetic/Binary Encryption scheme on the challenge $\left(x_{0}, x_{1}\right)$.

3. $\left|\operatorname{Pr}\left[\left\langle\mathcal{A}, G_{3}\right\rangle=1\right]-\operatorname{Pr}\left[\left\langle\mathcal{A}, G_{4}\right\rangle=1\right]\right|=\operatorname{neg}(n)$, else one could break the CPA security of the binary encryption scheme on the challenge $\left(0^{\ell}, e\right)$.

It follows, by a hybrid argument, that $\left|\operatorname{Pr}\left[\left\langle\mathcal{A}, G_{1}\right\rangle=1\right]-\operatorname{Pr}\left[\left\langle\mathcal{A}, G_{4}\right\rangle=1\right]\right|=\operatorname{neg}(n)$, and so $\mathcal{A}$ 's wining probability in the original IND-CPA game is $1 / 2 \pm \operatorname{neg}(n)$.

Remark 10.10 (Encrypting long messages). Construction 10.8 allows to encrypt a single field element. However, since we proved that the scheme is CPA-secure, it can be extended to encrypt a sequence of field elements via standard concatenation as explained in Remark 4.3.

By combining the above with Corollary 10.5, we derive a construction of CPA-secure public-key arithmetic encryption scheme based on the RLC assumption and on a standard binary publickey encryption scheme. A similar result holds in the symmetric setting, except that in this case the existence of binary CPA-secure encryption scheme already follows from the existence of (standard) one-way functions [GGM86, HILL99] which in turn follows from the RLC assumption (see Remark 10.6). Overall, we obtain the following corollary.

Corollary 10.11. Assuming $\operatorname{RLC}\left(n, \ell=\frac{(1+\varepsilon) n}{1-p}, p\right)$ holds for some constant $p \in(0,1)$ and constant $\varepsilon>0$, there exists a CPA-secure arithmetic symmetric encryption. Furthermore, if we additionally assume the existence of standard public-key encryption scheme, then there exists a CPA-secure arithmetic public-key encryption scheme.

\subsection{Direct Construction of Symmetric Encryption}

We proceed with a direct construction of symmetric encryption which generalizes the LPN-based construction of GRS08], see also [ACPS09]. Although the underlying assumption is worst than the one used in Corollary 10.11, the scheme demonstrates our claim that LPN-based encryption arithmetize.

Construction 10.12 (CPA-Secure Symmetric Encryption Scheme). We parameterize our construction by the constants $p \in\left(0, \frac{1}{2}\right)$ and $\varepsilon \in\left(0, \frac{1}{2}-p\right)$. For a security parameter $n$, we set the length parameter $\ell:=\left\lceil\frac{n p}{\varepsilon^{2}}\right\rceil$. The scheme consists of the following circuits:

- $\mathrm{KGen}_{n}$ : Sample $s \stackrel{R}{\leftarrow} \mathbb{F}^{n}$, and let $\mathrm{sk}=\mathrm{pk}=s$.

- $\operatorname{Enc}(s, x): M \stackrel{R}{\leftarrow} \mathbb{F}^{n \times n}, e \stackrel{R}{\leftarrow} \chi_{p}^{n}$, output $\left(M, c:=M s+e+x^{n}\right)$ where $x^{n}$ denotes the $n$-long column vector $(x, \ldots, x)$.

- $\operatorname{Dec}(s,(M, c))$ : Output the majority among the entries of the vector $c-M s$. 
Note that the majority operation can be computed arithmetically via the use of zerocheck gates and by describing logical majority via arithmetic gates (e.g., by going through the standard AND, NOT Boolean basis and then replacing it with multiplication and subtraction). Also, as explained in Section 8, there exists an arithmetic circuit that samples from $\chi_{p}^{\ell}$, and so the construction is realizable in the arithmetic model.

Remark. As we require correctness to hold for all fields, we need to choose $p+\varepsilon<1 / 2$ as otherwise, correctness would not hold over GF(2). Note that in settings where we are only interested in correctness for large fields we can replace the restriction $p<1 / 2$ with $p<1$ and use a ReedSolomon (RS) error-correcting code instead of the repetition code. (RS codes can be encoded and decoded arithmetically.) This modification allows to base the construction on a seemingly more secure assumption (with larger noise level). One can also improve the rate and efficiency of the scheme by using RS codes and by standard amortization techniques similar to the ones described in ACPS09.

Lemma 10.13 (Security). Suppose that the $\operatorname{RLC}(n, \ell, p)$ assumption holds for every polynomial $\ell(n)$ and for some constant $p \in(0,1 / 2)$. Then Construction 10.12 (instantiated with the same $p$ ) is computationally secure.

Proof. Assume towards a contradiction that an efficient adversary $\mathcal{A}$ wins the IND-CPA game with probability $1 / 2+\delta(n)$ for some non-negligible function $\delta$. Let $t=\operatorname{poly}(n)$ be an upper bound on the running-time of $\mathcal{A}$. We define a new adversary $\mathcal{B}$ against the RLC assumption in the following way:

\section{Adversary $\mathcal{B}$ :}

- Initiate $\mathcal{A}$ and get $\mathbb{F}$ from it. Send the same $\mathbb{F}$ to the challenger.

- Receive from the challenger $(M, v) \in\left(\mathbb{F}^{t \cdot n \times n}, \mathbb{F}^{t \cdot n}\right)$

- Parse $M=\left(M_{0}, \ldots, M_{t}\right)$ and $v=\left(v_{0}, \ldots, v_{t}\right)$ where $M_{i} \in \mathbb{F}^{n \times n}$ and $v_{i} \in \mathbb{F}^{n}$.

- For the $i$-th chosen-plaintext query of $\mathcal{A}$ :

- Send to $\mathcal{A}$ the pair $\left(M_{i}, v_{i}+x^{n}\right)$.

- For the challenge $x_{0}, x_{1}$ :

- Sample $b \in\{0,1\}$.

- Send to $\mathcal{A}$ the challenge $\left(M_{t}, v_{t}+x_{b}^{n}\right)$.

- If $\mathcal{A}$ wins, return 0 , else return 1 .

If the input $(M, v)$ is sampled from the RLC distribution $\mathcal{D}_{\mathbb{F}, p}^{t n \times n}$, then, by assumption, $\mathcal{A}$ wins with the probability $\frac{1}{2}+\delta(n)$, and $\mathcal{B}$ outputs 0 . However, if $(M, v) \stackrel{R}{\leftarrow}\left(\mathbb{F}^{t n \times n}, \mathbb{F}^{t n}\right)$, then the message is information-theoretically hidden from the adversary, and so $\mathcal{A}$ outputs 0 with probability exactly $1 / 2$. Therefore, $\mathcal{B}$ distinguishes $\mathcal{D}_{\mathbb{F}, p}^{t n \times n}$ from $\left(\mathbb{F}^{t n \times n}, \mathbb{F}^{t n}\right)$, contradicting our assumption.

Lemma 10.14 (Correctness). For the parameters as defined in Construction 10.12, for every field $\mathbb{F}$, it holds that

$$
\operatorname{Pr}\left[\operatorname{Dec}_{n}^{\mathbb{F}}\left(\operatorname{Enc}_{n}^{\mathbb{F}}(x, s), s\right)=x\right] \geq 1-\operatorname{neg}(n)
$$


Proof. Decryption fails only if the fraction of noisy coordinates in the vector $e \stackrel{R}{\leftarrow} \chi_{p}^{\ell}$ is larger than $\frac{1}{2}$. Since the constant $p$ is strictly smaller than $\frac{1}{2}$, by a Chernoff bound, the latter event happens with probability at most $\exp ^{-\Omega(n)}$.

Corollary 10.15. Let $p \in\left(0, \frac{1}{2}\right)$ be a constant, and assume $\operatorname{RLC}(n, \ell, p)$ holds for every polynomial $\ell(n)$, then there exists an IND-CPA secure symmetric encryption scheme in the arithmetic model.

\subsection{Direct Construction of Public Key Encryption}

In this section, we present a direct construction of an arithmetic public-key encryption scheme based on a variant of Alekhnovich's (binary) cryptosystem [Ale03. Again, the underlying RLC assumption is worst than the one used in Corollary 10.11, and the scheme is given in order to show that LPN-based encryption arithmetize.

Construction 10.16 (CPA-Secure Public Key Encryption Scheme). For a security parameter $n$, let $p=\frac{1}{2 \sqrt{n}}, \ell=2 n$.

- $\mathrm{KGen}_{n}$ :

- Sample $A \stackrel{R}{\leftarrow} \mathbb{F}^{\ell \times n}, s \stackrel{R}{\leftarrow} \mathbb{F}^{n}, e \stackrel{R}{\leftarrow} \chi_{p}^{\ell}$.

- Compute $b=A s+e$. Let $M=(b \mid A)$ where $\mid$ denotes column concatenation.

- If $\operatorname{Rank}(M) \neq(n+1)$ restart.

- Sample a random $\ell \times(\ell-n-1)$ matrix $B$ which spans $\operatorname{ker}\left(M^{T}\right){ }^{19}$ that is, $M B=$ $0^{(n+1) \times(\ell-n-1)}$.

- Let: $\mathrm{pk}=B, \mathrm{sk}=e$

- Enc $(\mathrm{pk}, x):$ Sample $s^{\prime} \stackrel{R}{\leftarrow} \mathbb{F}^{\ell-n-1}, e^{\prime} \stackrel{R}{\leftarrow} \chi_{p}^{\ell}$. Output: $c=B s^{\prime}+x^{\ell}+e^{\prime}$, recall that $x^{\ell}$ is an $\ell$ long vector where all the entries equal $x$.

- Dec(sk, c): If $\sum_{i=1}^{\ell} e_{i}=0$ output Fail. Else, compute: $x^{\prime}=\frac{e^{T} \cdot c}{\sum_{i=1}^{\ell} e_{i}}$ and output $x^{\prime}$.

Lemma 10.17 (Correctness). For every field $\mathbb{F}$, and for all $x \in \mathbb{F}$ :

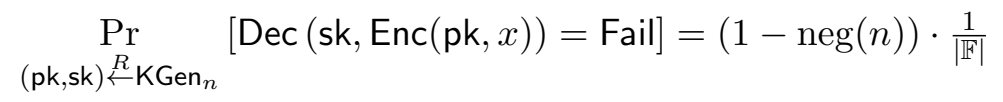

Conditioned on not failing, the probability of successful decryption is

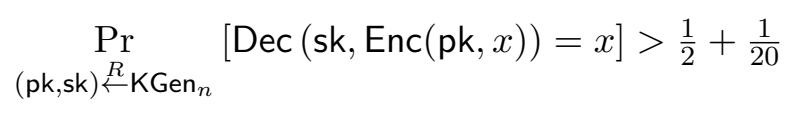

Proof. We start by showing (19). The decryption algorithm outputs Fail iff $\sum_{i=1}^{\ell} e_{i}=0$. With probability $(1-\operatorname{neg}(n))$, the vector $e$ includes at least one noisy coordinate, in which case the probability that $\sum_{i=1}^{\ell} e_{i}=0$ is exactly $1 /|\mathbb{F}|$.

We now turn to show (20). Conditioned on the event that decryption did not output Fail, the decryption algorithm solves the equation $x=\frac{e^{T} \cdot c}{\sum e_{i}}$. Expanding yields

$$
e^{T} c=e^{T} B s^{\prime}+e^{T} x^{\ell}+e^{T} e^{\prime} .
$$

\footnotetext{
${ }^{19}$ Such a B can be sampled by first finding any matrix that spans $\operatorname{ker}\left(M^{T}\right)$ and then multiplying it by a random invertible matrix.
} 
Notice that $e^{T} B s^{\prime}=0$, because $e^{T} \in \operatorname{Im}\left(M^{T}\right)$ and thus $e^{T} B=0^{\ell-n-1}$. Also observe that $\operatorname{Pr}_{e, e^{\prime}}\left[e^{T} e^{\prime}=0\right]>\frac{1}{2}+\frac{1}{20}$, because

$$
\operatorname{Pr}_{e, e^{\prime}}\left[e^{T} e^{\prime}=0\right] \geq\left(1-p^{2}\right)^{\ell}=\left(1-\frac{1}{4 n}\right)^{2 n}>\frac{1}{2}+\frac{1}{20} .
$$

Therefore, with probability greater than $\frac{1}{2}+\frac{1}{20}$ it holds that $e^{T} \cdot c=e^{T} \cdot x^{\ell}=x \sum_{i=1}^{\ell} e_{i}$ and decryption is successful.

Denote the above scheme as PKE. In order to prove security we define a variant of PKE, denoted $\mathrm{PKE}^{\prime}$, in which the public key $B \stackrel{R}{\leftarrow} \mathbb{F}^{\ell \times(n-1)}$, and Enc is defined as in PKE. Although we did not define a decryption algorithm for $\mathrm{PKE}^{\prime}$, its CPA-security is still well-defined. Our proof will now proceed as follows. Under the RLC assumption, we will first prove that $\mathrm{PKE}^{\prime}$ is semantically secure (Claim 10.18). We will then show that for every efficient field family $\mathbb{F}=\left\{\mathbb{F}_{n}\right\}$ it holds that pk $\stackrel{R}{\leftarrow} \mathrm{KGen}_{n}^{\mathbb{F}}$ is computationally indistinguishable from pk $\stackrel{R}{\leftarrow} \mathbb{F}^{\ell \times(n-1)}$ (Claim 10.19), and conclude that Construction 10.16 is semmantically secure (Lemma 10.20.

Claim 10.18. Suppose that $\operatorname{RLC}(n-1, \ell, p)$ holds for $\ell=2 n$ and $p=\frac{1}{2 \sqrt{n}}$, then $\mathrm{PKE}^{\prime}$ is semantically secure.

Proof. Assume towards a contradiction that an efficient adversary $\mathcal{A}$ wins the IND-CPA-game against the security of $\mathrm{PKE}^{\prime}$ with probability $1 / 2+\delta(n)$ for some non-negligible function $\delta$. We define a new adversary $\mathcal{B}$ against the $\operatorname{RLC}\left(n-1,2 n, \frac{1}{2 \sqrt{n}}\right)$ assumption in the following way:

\section{Adversary $\mathcal{B}$ :}

- Initiate $\mathcal{A}$ and get $\mathbb{F}$ from it. Send the same $\mathbb{F}$ to the challenger.

- Receive from the challenger $(B, v) \in\left(\mathbb{F}^{\ell \times(n-1)}, \mathbb{F}^{\ell}\right)$

- Send the public key $B$ to $\mathcal{A}$.

- When $\mathcal{A}$ requests an encryption of $x$ :

- Send $\operatorname{Enc}(B, x)$ to $\mathcal{A}$.

- For the challenge $x_{0}, x_{1}$ :

- Sample $b \in\{0,1\}$.

- Send $\left(v+x_{i}^{\ell}\right)$ to $\mathcal{A}$.

- If $\mathcal{A}$ wins (i.e. $\mathcal{A}$ outputs $b^{\prime}=b$ ) return 0 , else return 1 .

When $(B, v) \stackrel{R}{\leftarrow}\left(\mathbb{F}^{\ell \times(n-1)}, \mathbb{F}^{\ell}\right)$, then the information-theoretic security of the one time pad guarantees that $\mathcal{A}$ 's winning probability is exactly $1 / 2$, hence $\mathcal{B}$ wins with probability of $1 / 2$. However, when $(B, v) \stackrel{R}{\leftarrow} \mathcal{D}_{\mathbb{F}, p}^{\ell \times(n-1)}$ the vector $v+x_{i}^{\ell}$ is identically distributed as $\operatorname{Enc}\left(B, x_{i}\right)$, and so by assumption $\mathcal{A}$ wins the game with probability $1 / 2+\delta(n)$ and so does $\mathcal{B}$. Overall $\mathcal{B}$ wins the distinguishing game of the RLC assumption with probability $\frac{1}{2}+\frac{\delta(n)}{2}$, which contradicts the $\operatorname{RLC}\left(n-1,2 n, \frac{1}{2 \sqrt{n}}\right)$ assumption.

Claim 10.19 $\left(\mathrm{PKE} \approx \mathrm{PKE}^{\prime}\right)$. Suppose that $\operatorname{RLC}\left(n, \ell=2 n, p=\frac{1}{2 \sqrt{n}}\right)$ holds, then for every efficient adversary $\mathcal{A}$ the probability of winning the following game is at most $\frac{1}{2}+\operatorname{neg}(n)$ : 


\section{IND-Game $\left(1^{n}\right)$ :}

- $\mathcal{A}$ receives $1^{n}$, chooses a field $\mathbb{F}$ and sends $\mathbb{F}$ to the challenger.

- Challenger samples $\left(B_{0}, e\right) \stackrel{R}{\leftarrow} \mathrm{KGen}_{n}^{\mathbb{F}}$ as defined in Construction 10.16, and $B_{1} \stackrel{R}{\leftarrow}$ $\mathbb{F}^{\ell \times(n-1)}$.

- Challenger samples $b \in\{0,1\}$ and sends $B_{b}$ to $\mathcal{A}$.

- $\mathcal{A}$ outputs $b^{\prime}$ and wins if $b=b^{\prime}$.

Proof. In order to prove the claim we introduce two distributions: Let $\mathcal{U}_{\mathrm{rk}=n}^{\ell \times n}$ be the uniform distribution over the set of all matrices in $\mathbb{F}^{\ell \times n}$ with full rank $(\ell>n)$, and let $\mathcal{V}^{\ell \times n}$ be the distribution created by the following process: Sample $M \stackrel{R}{\leftarrow} \mathcal{U}_{\mathrm{rk}=(\ell-n)}^{\ell \times(\ell-n)}$, sample a random basis $B$ of $\operatorname{ker}\left(M^{T}\right)$, and output $B$.

The following two facts were proved in [Fel07] for the binary case, however they extend also to general fields:

1. Let $M \stackrel{R}{\leftarrow} \mathbb{F}^{\ell \times n}$ and $M^{\prime} \stackrel{R}{\leftarrow} \mathcal{U}_{\mathrm{rk}=n}^{\ell \times n}$. The statistical distance $\Delta\left(M, M^{\prime}\right) \leq|\mathbb{F}|^{-\ell+n}$.

2. Let $M \stackrel{R}{\leftarrow} \mathbb{F}^{\ell \times n}$ and $M^{\prime} \stackrel{R}{\leftarrow} \mathcal{V}^{\ell \times n}$. The statistical distance $\Delta\left(M, M^{\prime}\right) \leq|\mathbb{F}|^{-\ell+n}$.

We now turn to prove the claim. For any efficient distinguisher $\mathcal{A}$ attempting the game let $\mathbb{F}=\left\{\mathbb{F}_{n}\right\}$ be the efficient field family it outputs. We now use a hybrid argument to show that $\mathcal{A}$ wins the game with probability no more than $\frac{1}{2}+\operatorname{neg}(n)$. We define 4 variants of the key-generation algorithm, where the first hybrid corresponds to the real KGen and the last hybrid corresponds to the case where the key is random, see table 3.

\begin{tabular}{|l|l|l|l|l|}
\hline $\mathrm{KGen}_{n}$ & $\mathcal{H}_{1}$ & $\mathcal{H}_{2}$ & $\mathcal{V}^{\ell \times(n-1)}$ & $\mathbb{F}^{\ell \times(n-1)}$ \\
\hline$A \stackrel{R}{\leftarrow} \mathbb{F}^{\ell \times n}, s \stackrel{R}{\leftarrow} \mathbb{F}^{n}, e \stackrel{R}{\leftarrow} \chi_{p}^{\ell}, b=A s+e$ & $b \stackrel{R}{\leftarrow} \mathbb{F}^{\ell}$ & & \\
$M=(b \mid A)$, conditioned on $\operatorname{Rank}(M)=n+1$ & no rank condition & & $M \stackrel{R}{\leftarrow} \mathcal{U}_{\mathrm{rk}=n+1}^{\ell \times n+1}$ & \\
$B \stackrel{R}{\leftarrow}$ a random matrix that spans $\operatorname{ker}\left(M^{T}\right)$ & & & & \\
\hline \multicolumn{2}{|l|}{ output: $B$} & & & \\
\hline
\end{tabular}

Table 3: The hybrid distributions. $\mathbb{F}$ is the field family chosen by $\mathcal{A}\left(1^{n}\right)$. For readability, only the modifications with respect to the previous hybrid distribution appear.

- $\mathrm{KGen}_{n} \stackrel{c}{\approx} \mathcal{H}_{1}$ : Assume $b \stackrel{R}{\leftarrow} \mathbb{F}^{\ell}$. Then by Fact (1) for $\ell=2 n$ we get that $\operatorname{Pr}[\operatorname{Rank}(M) \neq$ $(n+1)]=\operatorname{neg}(n)$. If this does not holds for $(A, b) \stackrel{R}{\leftarrow} \mathcal{D}_{\mathbb{F}, p}^{\ell \times n}$, then a rank test is an immediate efficient adversary against the $\operatorname{RLC}\left(n, 2 n, \frac{1}{2 \sqrt{n}}\right)$ assumption ${ }^{20}$

- $\mathcal{H}_{1} \stackrel{c}{\approx} \mathcal{H}_{2}$ : A distinguisher between the two distributions immediately implies an adversary that wins the IND-game of $\operatorname{RLC}\left(n, 2 n, \frac{1}{2 \sqrt{n}}\right)$ over the same field family with the same advantage.

- $\mathcal{H}_{2} \stackrel{s}{\approx} \mathcal{V}^{\ell \times(n-1)}$ : In $\mathcal{H}_{2}$, we have that $M \stackrel{R}{\leftarrow} \mathbb{F}^{\ell \times(n+1)}$ and therefore the claim follows from Fact (1) since $\ell=2 n$.

\footnotetext{
${ }^{20}$ In this case, one can actually show statistical indistinguishability, but for our purposes computational indistinguishability suffices.
} 
- $\mathcal{V}^{\ell \times(n-1)} \stackrel{s}{\approx} \mathbb{F}^{\ell \times(n-1)}$ : Follows from Fact $(2)$ for $\ell=2 n$.

By a hybrid argument for the field family $\mathbb{F}$, The ensemble $\mathrm{KGen}_{n}$ is computationally indistinguishable from the uniform distribution over $\mathbb{F}_{n}^{\ell \times(n-1)}$, hence $\mathcal{A}$ can win the distinguishing game with probability no more than $\frac{1}{2}+\operatorname{neg}(n)$.

We can now prove the security of Construction 10.16 .

Lemma 10.20 (Security). Suppose that $\operatorname{RLC}(n-1, \ell, p)$ holds for $\ell=2 n$ and $p=\frac{1}{2 \sqrt{n}}$, then Construction 10.16 is CPA-secure.

Proof. Assume towards contradiction that adversary $\mathcal{A}$ wins the IND-CPA-game against Construction 10.16 with probability $\frac{1}{2}+\delta(n)$ for a non negligible $\delta$. Consider the following adversary $\mathcal{B}$ which distinguishes a random Alekhnovich pk from a random matrix.

\section{Adversary $\mathcal{B}$ :}

- Initiate $\mathcal{A}$ and get $\mathbb{F}$ from it. Send the same $\mathbb{F}$ to the challenger.

- Receive from the challenger $B \in \mathbb{F}^{\ell \times(n-1)}$

- Send the public key $B$ to $\mathcal{A}$.

- When $\mathcal{A}$ requests an encryption of $m$ :

- Send $\operatorname{Enc}(B, x)$ to $\mathcal{A}$.

- For the challenge $x_{0}, x_{1}$ :

- Sample $b \in\{0,1\}$.

- Send $\operatorname{Enc}\left(B, x_{b}\right)$ to $\mathcal{A}$.

- If $\mathcal{A}$ wins return 0 , else return 1 .

By Claim 10.18 if $B \stackrel{R}{\leftarrow} \mathbb{F}^{\ell \times(n-1)}$, then $\mathcal{A}$ wins (and $\mathcal{B}$ looses) with probability no more than $\frac{1}{2}+\operatorname{neg}(n)$. By assumption when $B \stackrel{R}{\leftarrow} \operatorname{KGen}_{n}^{\mathbb{F}}$ the adversary $\mathcal{A}$ wins (and $\mathcal{B}$ wins) wins with probability $\frac{1}{2}+\delta(n)$ for a non-negligible $\delta$. Hence overall $\mathcal{B}$ wins the IND-game of Claim 10.19 with probability $\frac{1}{2}+\frac{\delta(n)-n e g(n)}{2}$, a non-negligible advantage. To prove the lemma (via Claim 10.19 it suffices to show that $\operatorname{RLC}\left(n, \ell=2 n, p=\frac{1}{2 \sqrt{n}}\right)$ is hard.

Recall that we only assumed the hardness of $\operatorname{RLC}\left(n-1,2 n, \frac{1}{2 \sqrt{n}}\right)$ and not $\operatorname{RLC}\left(n, 2 n, \frac{1}{2 \sqrt{n}}\right)$, however this is not a real issue as $\operatorname{RLC}(n-1, \ell, p)$ implies $\operatorname{RLC}(n, \ell, p)$ (for any $\ell$ and $p$ ). The reduction is as follows: For $(M, v)$ sample a random $s \in \mathbb{F}$ and $x \in \mathbb{F}^{\ell}$, and return $((M \mid x), v+s x)$. It can be seen that for $(M, v) \stackrel{R}{\leftarrow} \mathcal{D}_{\mathbb{F}, p}^{\ell \times n-1}$ then $((M \mid x), v+s x) \stackrel{R}{\leftarrow} \mathcal{D}_{\mathbb{F}, p}^{\ell \times n}$, and for $(M, v) \stackrel{R}{\leftarrow}\left(\mathbb{F}^{\ell \times(n-1)}, \mathbb{F}^{\ell}\right)$, $((M \mid x), v+s x) \stackrel{R}{\leftarrow}\left(\mathbb{F}^{\ell \times n}, \mathbb{F}^{\ell}\right)$. Therefore a distinguisher for $\operatorname{RLC}(n, \ell, p)$ implies a distinguisher for $\operatorname{RLC}(n-1, \ell, p)$.

Building on Lemma 10.17 and Lemma 10.20 we can use amplification to create a PKE scheme with overwhelming success probability in the following way. We repeat the above construction, each time with fresh keys, polynomially many times. The decryption algorithm then uses majority to decide which message to output. As Fail occurs over any field with probability no more than $1 / 2$ with overwhelming probability there will be polynomially many non-failed decryptions, for which again by Chernoff's inequality with overwhelming probability the correct $m$ will be the majority. Security will not be harmed as each execution uses fresh randomness. 
Corollary 10.21. Assuming $\operatorname{RLC}(n-1, \ell, p)$ holds for $\ell=2 n$ and $p=\frac{1}{2 \sqrt{n}}$ then there exists a CPA-secure public key encryption scheme in the arithmetic model.

Again, we emphasize that Corollary 10.11 provides arithmetic PKE under a weaker (and therefore better) assumption.

\section{Commitments}

In this section we give candidate constructions for statistically binding string commitment schemes under RLC assumptions. We give two different constructions. In Section 11.1 we give a non interactive construction in the Common Reference String model where we assume the key generation is done by some trusted party. The advantage of this construction is that it carries some homomorphic properties. In Section 11.2 we give a construction in the standard model by showing that the PRG-based construction of Naor [Nao91] arithmetize, and by plugging-in an arithmetic PRG based on the RLC assumption.

\subsection{Non-Interactive Statistically Binding String Commitment}

Non-interactive statistically-binding string commitment schemes based on the hardness of learning parity with noise were given in [AIK10, JKPT12]. Using the RLC assumption, we generalize their schemes to the arithmetic setting and build a commitment scheme for tuples of field elements.

Our construction is in the CRS model where we assume that KGen is executed by some trusted party. For the definition of a commitment scheme see Definition 4.4 .

Construction 11.1 (Statistically Binding String Commitment). The commitment scheme is parameterized by the security parameter $n \in \mathbb{N}$, a noise parameter $p \in(0,1 / 8)$, a length parameter $\ell=$ cn where $c=\left\lceil\frac{2}{1-H_{2}(4 p)-\varepsilon}\right\rceil$, and an arbitrarily small constant $\varepsilon \in\left(0,1-H_{2}(4 p)\right)$. Set $w=\lfloor\ell p\rceil$. The algorithms of the commitment scheme are as follows:

- $\mathrm{KGen}_{n}^{\mathbb{F}}$ : The public commitment key consists of the matrix $A=\left(A^{\prime} \mid A^{\prime \prime}\right) \in \mathbb{F}^{\ell \times(2 n)}$, where $A^{\prime} \stackrel{R}{\leftarrow} \mathbb{F}^{\ell \times n}$ and $A^{\prime \prime} \stackrel{R}{\leftarrow} \mathbb{F}^{\ell \times n}$.

- $\operatorname{Com}_{n}^{\mathbb{F}}(A, x)$ : To commit to a message $x \in \mathbb{F}^{n}$ using the public key A, the Sender samples $r \stackrel{R}{\leftarrow} \mathbb{F}^{n}$ and $e \stackrel{R}{\leftarrow} \chi_{p}^{\ell}$, and computes the commitment $c=A \cdot(r \mid x)+e$. The decommitment of the commitment is the pair $(x, r)$.

- $\operatorname{Ver}_{n}^{\mathbb{F}}\left(A, c,\left(x^{\prime}, r^{\prime}\right)\right)$ : Given a public key $A$, a commitment $c$ and a decommitment $\left(x^{\prime}, r^{\prime}\right)$, the Receiver computes $e^{\prime}=c-A \cdot\left(r^{\prime} \mid x^{\prime}\right)$ and outputs 1 iff $w\left(e^{\prime}\right) \leq 2 w$.

The constant $c$ was chosen to satisfy the following inequality for every integer $q \geq 2$ :

$$
c=\left\lceil\frac{2}{1-H_{2}(4 p)-\varepsilon}\right\rceil \geq\left\lceil\frac{2}{1-H_{q}(4 p)-\varepsilon}\right\rceil>2,
$$

where $H_{q}(\alpha):=-\alpha \log _{q}(\alpha)-(1-\alpha) \log _{q}(1-\alpha)$ denotes the $q$-ary entropy function. To see that Eq. 21 holds, recall that $p \in(0,1 / 8)$, and so $4 p \in(0,1 / 2)$. Since for every $\alpha \in(0,1 / 2)$ we have that $H_{2}(\alpha) \geq H_{q}(\alpha)>0$ the equation follows. 
We turn now to establishing correctness, that is, that in an honest execution, the verifier accepts with overwhelming probability. If the sampled vector $e \stackrel{R}{\leftarrow} \chi_{p}^{\ell}$ is such that $w(e) \leq 2 w$, then the verification succeeds with probability 1 . By Chernoff's inequality for $p \in(0,1 / 8)$ and $\ell=c n$ (for any constant $c$ )) the probability that the weight of $e$ exceeds $2 w$ is negligible in $n$. We now turn to security.

Lemma 11.2. Suppose that the $\operatorname{RLC}(n, \ell, p)$ assumption holds for some constant $p \in(0,1 / 8)$, and for $\ell=\left\lceil\frac{2 n}{1-H_{2}(4 p)-\varepsilon}\right\rceil$. Then Construction 11.1 is a statistically binding and computationally hiding commitment scheme.

Proof. We start by stating a fact about random linear codes. For a random linear code with a generating matrix $M \stackrel{R}{\leftarrow} \mathbb{F}^{\ell \times n}$, for $\delta \in\left(0,1-\frac{1}{|\mathbb{F}|}\right)$, when $\ell \geq \frac{n}{1-H_{|\mathbb{F}|}(\delta)-\varepsilon}$ the probability that the error correcting code defined by $M$ has a minimum distance smaller than $\delta \ell$ is no more than $|\mathbb{F}|^{-\varepsilon \ell}$. For a proof we refer the reader to chapter 4 of [VGS14.

Instantiating our parameters of $p$ and $\ell$ in the above fact and combining with (21), and setting $\delta=4 p$, and $\ell=c n$ we get that over any field $\mathbb{F}$ with overwhelming probability the minimum distance of the code generated by $A$ is more than $4 \ell p$.

We can now prove that the protocol is statistically binding. Assume towards contradiction that $x_{i}, r_{i}, i=1,2$ are two different decommitments for the same commitment $c$. Then we have that $e_{i}=c-A\left(r_{i} \mid x_{i}\right)$ has a weight of no more than $2 w$. Hence $e_{1}-e_{2}=A\left(r_{1}-r_{2} \mid x_{1}-x_{2}\right)$ is a codeword of weight less than $4 w$, contradicting the previous observation on the minimal weight of a codeword generated by $A$.

Finally, we prove that the commitment scheme is computationally hiding. Assume that an adversary $\mathcal{A}$ breaks the hiding property and wins the indistinguishability game with some nonnegligible advantage $\delta$. Consider the following adversary against the RLC assumption:

\section{Adversary $\mathcal{B}\left(1^{n}\right)$ :}

- Initiate $\mathcal{A}\left(1^{n}\right)$ and get $\mathbb{F}$ from it. Send the same $\mathbb{F}$ to the challenger.

- Receive from the challenger $\left(A^{\prime}, u\right) \in\left(\mathbb{F}^{\ell \times n}, \mathbb{F}^{\ell}\right)$.

- Sample $A^{\prime \prime} \stackrel{R}{\leftarrow} \mathbb{F}^{\ell \times n}$ and send pk $=\left(A^{\prime} \mid A^{\prime \prime}\right)$ to the adversary $\mathcal{A}$.

- Receive from $\mathcal{A}$ a pair $\left(x_{0}, x_{1}\right) \in\left(\mathbb{F}^{n}, \mathbb{F}^{n}\right)$.

- Sample $b \in\{0,1\}$, compute $c=u+A^{\prime \prime} \cdot x_{b}$, and send $c$ to $\mathcal{A}$.

- Return 0 if $\mathcal{A}$ wins (i.e., $\mathcal{A}$ returns $b^{\prime}=b$ ), and return 1 otherwise.

Observe that if $\left(A^{\prime}, u\right) \stackrel{R}{\leftarrow}\left(\mathbb{F}^{\ell \times n}, \mathbb{F}^{\ell}\right)$ then $c \stackrel{R}{\leftarrow} \mathbb{F}^{\ell}$ and $\mathcal{A}$ wins with probability exactly $1 / 2$, hence $\mathcal{B}$ wins with probability exactly $1 / 2$. On the other hand, when $\left(A^{\prime}, u\right) \stackrel{R}{\leftarrow} \mathcal{D}_{\mathbb{F}, p}^{\ell \times n}$ then $(\mathrm{pk}, c) \stackrel{i}{\approx}$ (pk, $\operatorname{Com}_{\mathrm{pk}}^{\mathbb{F}}\left(x_{b}\right)$ ), and by assumption $\mathcal{A}$ wins (hence $\mathcal{B}$ wins) with probability $1 / 2+\delta$. Overall, $\mathcal{B}$ wins the IND-game against the RLC assumption with the non-negligible advantage $\delta / 2$, in contradiction to our hypothesis.

Corollary 11.3. Assuming the $\operatorname{RLC}(n, \ell, p)$ assumption holds for some constant $p \in(0,1 / 8)$, and for $\ell=\left\lceil\frac{2 n}{1-H_{2}(4 p)-\varepsilon}\right\rceil$, there exists an arithmetic commitment scheme in CRS model. 


\subsection{CRS Free Commitment Protocol}

We now introduce a commitment protocol that does not require a common reference string by providing an arithmetic analog of Naor's commitments [Nao91]. Syntactically, we still consider a non-interactive commitment function parameterized by a public-key which is generated by a keygeneration algorithm. However, the hiding property should hold for every (adversarially chosen) public-key. This gives rise to a two-message commitment protocol in the standard model (no CRS) in which the receiver chooses the public-key pk. The construction is based on an arithmetic pseudorandom generator (APRG) $\mathrm{APRG}_{n}$ which stretches $n$ inputs to $3 n$ outputs. In the following, we will write $\operatorname{APRG}_{n}(s)$ to denote explicitly the output of $\operatorname{APRG}_{n}(s)$ on a seed $s$. Note that the total length of $s$ is $n$ and it may be composed from both random field elements and random bits.

Construction 11.4 (CRS Free Statistically Binding Commitment Protocol). The commitment scheme is parameterized by a security parameter $n \in \mathbb{N}$. Let APRG be any arithmetic PRG with expansion factor 3. Define the protocol as follows:

- $\mathrm{KGen}_{n}^{\mathbb{F}}$ : The Receiver publishes the public key $r \stackrel{R}{\leftarrow} \mathbb{F}^{3 n}$.

- $\operatorname{Com}_{n}^{\mathbb{F}}(r, x):$ To commit to a message $x \in \mathbb{F}$ using the public key $r$, the Sender samples a seed $s$ for the $A P R G$ and computes $c=\operatorname{APRG}_{n}(s)+x \cdot r$. The decommitment is the pair $(s, x)$.

- $\operatorname{Ver}_{n}^{\mathbb{F}}\left(r, c,\left(s^{\prime}, x^{\prime}\right)\right)$ : Given a commitment $c$, a public key $r$, and a decommitment $\left(s^{\prime}, x^{\prime}\right)$, the Receiver outputs 1 iff $c=\operatorname{APRG}\left(s^{\prime}\right)+x^{\prime} \cdot r$.

We now prove that this protocol indeed realizes a commitment scheme.

Lemma 11.5. Assuming the pseudorandomness of APRG, the scheme is computationally hiding. Furthermore, this holds even if the public-key is chosen adversarially.

Proof. Assume that an efficient malicious receiver $\mathcal{R}$ can break the hiding property of the scheme. $\mathcal{R}$ chooses the field $\mathbb{F}$, and a public key $r$ for which it wins the distinguishing game in Definition 4.4 with probability $\frac{1}{2}+\delta(n)$ for some non-negligible $\delta$. Consider the following adversary against the security of the APRG:

\section{Adversary $\mathcal{A}$ :}

1. Invoke $\mathcal{R}$ and get $\mathbb{F}$ form it.

2. Get $r \in \mathbb{F}^{3 n}$ and $x_{0}, x_{1} \in \mathbb{F}$ from $\mathcal{R}$.

3. Initialize the distinguishing game against the APRG with the field $\mathbb{F}$ :

(a) Get $u \in \mathbb{F}^{3 n}$ from challenger.

(b) Sample $b \stackrel{R}{\leftarrow}\{0,1\}$ and send $\mathcal{R}$ the commitment $c=u+x_{b} \cdot r$.

(c) If $\mathcal{R}$ returns $b^{\prime}=b$ output 1 . Else output 0 .

By assumption when $u$ is a random output of the APRG, $c$ is distributed identically to an honestly generated commitment and $\mathcal{R}$ must win with probability $\frac{1}{2}+\delta(n)$ for some non-negligible $\delta$. Hence in this case, $\mathcal{A}$ returns 1 and wins also with the probability of $\frac{1}{2}+\delta(n)$. However when $u \stackrel{R}{\leftarrow} \mathbb{F}^{n}, c$ hides $b$ information theoretically, and therefore $\mathcal{R}$ wins with probability exactly $1 / 2$, and $\mathcal{A}$ returns 0 and wins with probability exactly $1 / 2$. Overall $\mathcal{A}$ wins with probability $\frac{1}{2}+\frac{\delta(n)}{2}$ in contradiction to the security of the APRG. 
Lemma 11.6. With overwhelming probability over $r \stackrel{R}{\leftarrow} \mathbb{F}^{3 n}$, the scheme is statistically binding.

Proof. Call a public-key $r \in \mathbb{F}^{3 n}$ ambiguous if there exist a pair of messages $x_{0} \neq x_{1}$, and a pair of seeds $s_{0}$ and $s_{1}$ for which APRG $\left(s_{0}\right)+x_{0} r=\operatorname{APRG}\left(s_{1}\right)+x_{1} r$. This happens if and only if $r=\frac{\operatorname{PRG}\left(s_{0}\right)-\operatorname{APRG}\left(s_{1}\right)}{x_{1}-x_{0}}$, however the right hand side consists of no more than $|\mathbb{F}|^{2 n+1}$ possible vectors, and so the probability that a random $r$ is ambiguous is no more than $|\mathbb{F}|^{2 n+1} /|\mathbb{F}|^{3 n}=|\mathbb{F}|^{-n+1}$, which is negligible.

Since an arithmetic PRG with expansion factor of three can be based on any APRG (Lemma 9.2), and since the latter can be based on the RLC assumption (Theorem 9.3), we obtain the following corollary.

Corollary 11.7. Assuming an arithmetic PRG, there exists an arithmetic commitment protocol that does not require a common reference string. Specifically, if $\operatorname{RLC}\left(n, \ell=\frac{n}{1-p-\varepsilon}, p\right)$ holds for some constants $p \in(0,1), \varepsilon>0$, then there exists an arithmetic commitment protocol that does not require a common reference string.

\section{Secure Computation}

In this section we show how to securely compute any two-party arithmetic functionality. We start with simple arithmetic functionalities such as $\left(\begin{array}{l}2 \\ 1\end{array}\right)$-Arithmetic Oblivious Transfer (Section 12.1), and Oblivious Linear Evaluation (Section 12.2), and eventually (Section 12.3) show how to privately compute any two party functionality that is described by an arithmetic circuit over addition and multiplication gates only. (The results extend to the multiparty setting in a straight forward way.) We will mostly focus in the semi-honest setting. An adaptation to the malicious model is sketched in Section 12.4.

\section{1 ( $\left(\begin{array}{l}2 \\ 1\end{array}\right)$-Arithmetic Oblivious Transfer}

We define $\left(\begin{array}{l}2 \\ 1\end{array}\right)$ Arithmetic Oblivious Transfer via the following partial functionality: the Receiver's input is a selection bit $x \in\{0,1\}$ and security parameter $1^{n}$ and the sender's input is a pair of field elements $z_{0}, z_{1} \in \mathbb{F}$ and security parameter $1^{n}$. The functionality delivers to the receiver the value $z_{x}$ (which can be written arithmetically as $x \cdot z_{1}+(1-x) z_{2}$ ). The sender receives no output (an empty string). The more standard binary variant of this functionality (denoted $\left(\begin{array}{l}2 \\ 1\end{array}\right)$-OT) corresponds to the case where the sender's inputs $z_{0}, z_{1}$ are binary strings (of $\operatorname{poly}(n)$ length).

We begin by showing how to use ABE to privately reduce $\left(\begin{array}{l}2 \\ 1\end{array}\right)$-AOT to standard binary $\left(\begin{array}{l}2 \\ 1\end{array}\right)$-OT. The construction is similar to the standard transformation from OT over short strings to OT over long strings.

Construction 12.1. Let OT be any binary (string) oblivious transfer protocol, and let $\mathcal{E}=$ (ABGen, ABEnc, ABDec) be any Arithmetic/Binary Encryption scheme. Let $z_{0}, z_{1} \in \mathbb{F}$ be the input of the sender and $x \in\{0,1\}$ the input of the receiver. Consider the following scheme:

- Sender samples $\mathrm{sk}_{0}, \mathrm{sk}_{1} \stackrel{R}{\leftarrow} \mathrm{ABGen}_{n}$.

- Both parties run the binary OT protocol with the input $\left(x,\left(\mathrm{sk}_{0}, \mathrm{sk}_{1}\right)\right)$ (recall that $\mathrm{sk}_{i}$ is a binary string). 
- Sender sends to receiver $c_{i} \stackrel{R}{\leftarrow} \mathrm{ABEnc}_{n}^{\mathbb{F}}\left(\mathrm{sk}_{i}, z_{i}\right)$ for $i \in\{0,1\}$.

- Receiver outputs $z_{x}=\operatorname{ABDec}_{n}^{\mathbb{F}}\left(\mathrm{sk}_{x}, c_{x}\right)$. In case decryption fails, outputs 1 .

Theorem 12.2. Assuming that $\mathcal{E}$ is an ABE, Construction 12.1 privately reduces $\left(\begin{array}{l}2 \\ 1\end{array}\right)$-AOT to standard binary $\left(\begin{array}{l}2 \\ 1\end{array}\right)$-OT.

In fact, it can be shown that, if the underlying binary OT is secure in the malicious setting, then so is the resulting $\left(\begin{array}{l}2 \\ 1\end{array}\right)$-AOT. (See Section 12.4.1.)

Proof. The correctness of Construction 12.1 follows immediately from the correctness of the binary $\mathrm{OT}$ and the correctness of the $A B E$. The simulator for the sender is trivial, as it gets no message. The simulator $\operatorname{Sim}_{2}\left(x, z_{x}\right)$ for the receiver generates a view which corresponds to messages sent by a Sender whose $x$-th input is $z_{x}$ and its other input $z_{1-x}$ is set to zero. Formally, $\operatorname{Sim}_{2}\left(x, z_{x}\right)$ samples $\mathrm{sk}_{0}, \mathrm{sk}_{1} \stackrel{R}{\leftarrow} \mathrm{ABGen}_{n}$, computes the ciphertexts $c_{i} \stackrel{R}{\leftarrow} \mathrm{ABEnc}_{n}^{\mathbb{F}}\left(\mathrm{sk}_{i}, z_{i}\right)$ where $z_{1-x}=0$ and outputs the tuple $\left(x, \mathbf{s k}_{x}, c_{0}, c_{1}\right)$. The security of the ABE guarantees that the simulated view is indistinguishable from the real view.

Combining the construction with Corollary 10.5, we derive the following corollary.

Corollary 12.3. Assume that for some constant $p \in(0,1)$ and constant $\varepsilon>0$ the $\operatorname{RLC}(n, \ell=$ $\left.\frac{(1+\varepsilon) n}{1-p}, p\right)$ assumption holds. Then, assuming the existence of binary Oblivious Transfer protocol, there exist an arithmetic $\left(\begin{array}{l}2 \\ 1\end{array}\right)$-AOT protocol.

The latter assumption (existence of binary Oblivious Transfer protocol) is necessary as an arithmetic $\left(\begin{array}{l}2 \\ 1\end{array}\right)$-AOT protocol immediately implies the existence of binary Oblivious Transfer.

\subsubsection{Alternative Construction based Alekhnovich's PKE}

An alternative construction of $\left(\begin{array}{l}2 \\ 1\end{array}\right)$-AOT can be established by observing that the public-key in Alekhnovich's encryption scheme is pseudorandom.

Construction 12.4. The protocol is described in Figure 2. Gen, Dec, Enc are the arithmetic circuits of the Alekhnovich PKE scheme, as defined in Construction 10.16.

Correctness of the scheme follows from the correctness of the underlying PKE scheme.

Lemma 12.5 (Privacy). Suppose that the $\operatorname{RLC}\left(n-1,2 n, \frac{1}{2 \sqrt{n}}\right)$ assumption holds, then Construction 12.4 privately realizes the $\left(\begin{array}{l}2 \\ 1\end{array}\right)$-AOT functionality.

Proof. Correctness follows from the correctness of Alekhnovich's PKE (Lemma 10.17). The simulator of the Sender $\operatorname{Sim}_{1}\left(z_{0}, z_{1}\right)$ simply outputs a pair of random matrices $M_{0}, M_{1} \stackrel{R}{\leftarrow} \mathbb{F}^{\ell \times n-1}$ (together with its inputs and its internal randomness). The pseudorandomness of public-keys (as established in Claim 10.19 shows that the simulated view is indistinguishable from the real view. The simulator of the Receiver $\operatorname{Sim}_{2}(x)$ essentially replaces $c_{1-x}$ with an encryption of zero. Formally, $\operatorname{Sim}_{2}\left(x, z_{x}\right)$ samples $\left(M_{x}, e\right) \stackrel{R}{\leftarrow} \mathrm{KGen}_{n}$ and $M_{1-x} \stackrel{R}{\leftarrow} \mathbb{F}^{\ell \times n-1}$ computes $c_{x}=\operatorname{Enc}_{n}\left(M_{x}, z_{x}\right)$ and $c_{1-x}=\operatorname{Enc}_{n}\left(M_{1-x}, 0\right)$ and outputs $\left(x, M_{0}, M_{1}, e, c_{0}, c_{1}\right)$. The semantic security of Alekhnovich's encryption over random keys (Claim 10.18) implies that the simulated view is indistinguishable from the real view. 


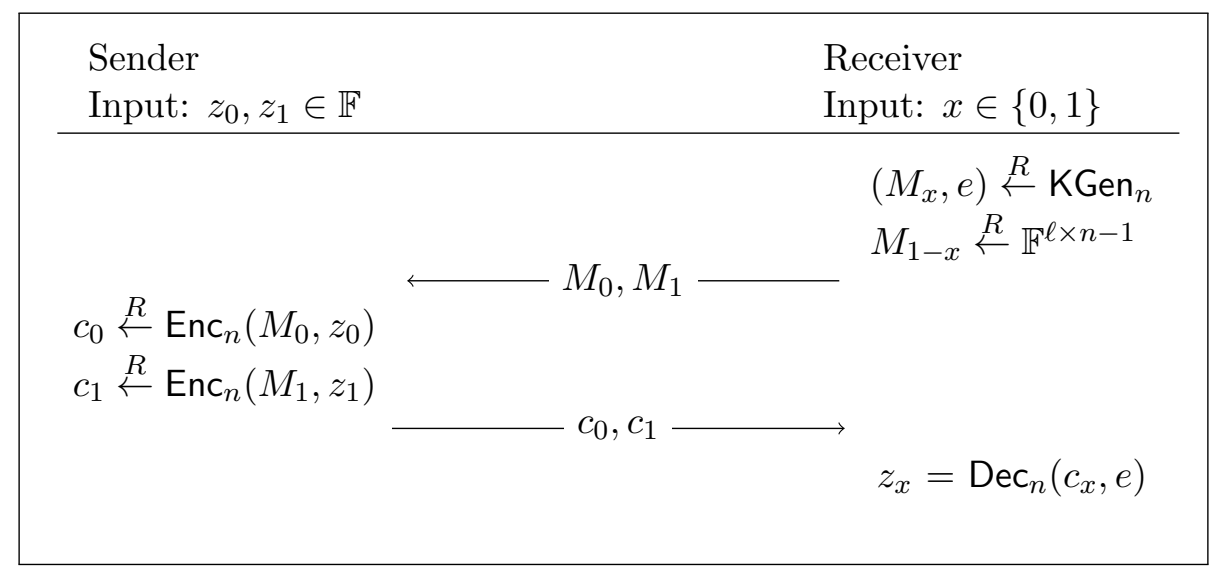

Figure 2: $\left(\begin{array}{l}2 \\ 1\end{array}\right)$-AOT from Alekhnovich

Corollary 12.6. Assuming $\operatorname{RLC}\left(n-1,2 n, \frac{1}{2 \sqrt{n}}\right)$, there exist a computationally secure $\left(\begin{array}{l}2 \\ 1\end{array}\right)$-AOT protocol in the semi-honest model.

\subsection{From $\left(\begin{array}{l}2 \\ 1\end{array}\right)$-AOT to Oblivious Linear Evaluation}

The Oblivious Linear Evaluation (OLE) functionality takes a single field element $x \in \mathbb{F}$ from the receiver and a pair of field elements $a, b \in \mathbb{F}$ from the sender and delivers to the receiver the value $a x+b$ (and nothing to the sender). As before, we assume that both parties are also given a common security parameter $1^{n}$.

OLE via (weakly) homomorphic encryption. A natural way to obtain OLE is via the use of a (weak) homomorphic encryption. Specifically, let the receiver encrypt $x \in \mathbb{F}$, and let the sender homomorphically modify the ciphertext $c=E_{k}(x)$ to $c^{\prime}=E_{k}(a x+b)$. Since the new ciphertext may leak information on $a$ and $b$ (as is the case in our RLC-based OLE) we will refresh the ciphertext $c^{\prime}$ via the use of a sub-protocol based on $\left(\begin{array}{l}2 \\ 1\end{array}\right)$-AOT. Below we instantiate this approach with the ABE from Construction 10.1. (See also Remark 12.9 for a generalization.)

Concretely, recall that we encrypted a message $x \in \mathbb{F}$ by sampling $(M, v)$ from some RLC distribution $(v=M s+e)$ and used $v$ to pad the (encoded) message $x^{\ell}$. Notice that such an encryption has weak homomorphic properties. Namely, given a ciphertext $(M, c)=\operatorname{Enc}(x)$ and scalars $a, b \in \mathbb{F}$ we can compute a new ciphertexts $\left(M, c^{\prime}=a \cdot c+b^{\ell}\right)$ which form a valid encryption of $a x+b$. While it is easy to re-randomize the vector $s$ (by adding $M \cdot s^{\prime}$ for some random $s^{\prime}$ ), the noisy coordinates remain correlated with the original noise vector. We remove this correlation by letting the receiver learn only the non-noisy coordinate using the $\left(\begin{array}{l}2 \\ 1\end{array}\right)$-AOT. The construction is given in Figure 3. A similar approach (under a different abstraction) appears in [IPS09.

Theorem 12.7. Assuming $\operatorname{RLC}\left(n, \ell:=\frac{(1+\varepsilon) n}{1-p}, p\right)$ for some constant $p \in(0,1)$ and constant $\varepsilon>0$, the construction in Figure 3 privately reduces OLE to $\left(\begin{array}{l}2 \\ 1\end{array}\right)$-AOT.

Proof. First observe that the $i$-th coordinate of $c^{\prime \prime}$ satisfies the equality

$$
c_{i}^{\prime \prime}=\left(a c+b^{\ell}+M r\right)_{i}=\left(M(a s+r)+(a x+b)^{\ell}\right)_{i} \quad \text { for } i: e_{i}=0,
$$




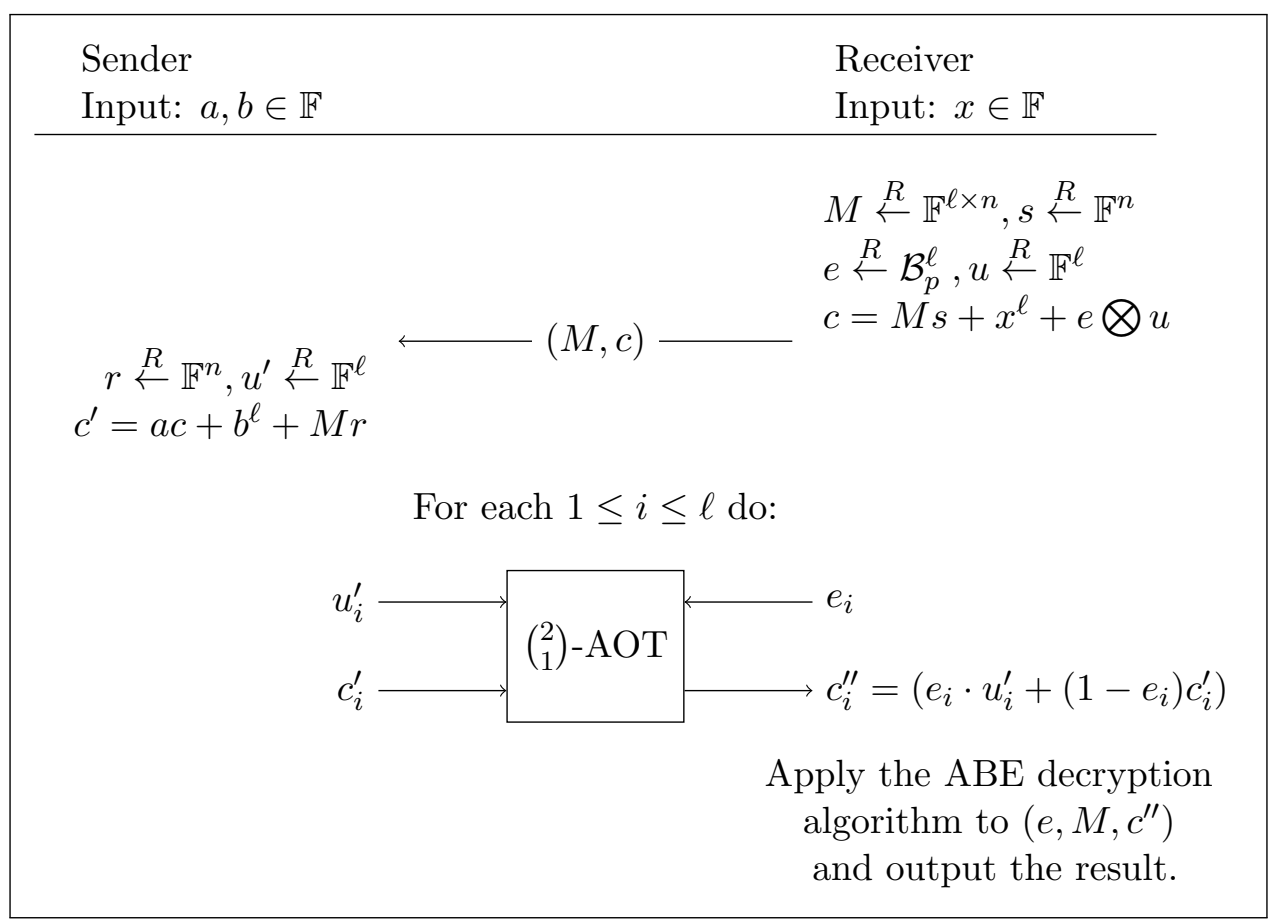

The notation $\otimes$ stands for entry-wise product.

Figure 3: OLE from $\left(\begin{array}{l}2 \\ 1\end{array}\right)$-AOT 
and $c_{i}^{\prime \prime}=u_{i}^{\prime}$ for $i: e_{i}=1$. Hence, $\left(M, c^{\prime \prime}\right)$ is distributed as a fresh encryption of $(a x+b)$ under the private-key $e$. Furthermore, this ciphertext is statistically independent of $s$ and $e$. Correctness now follows from the correctness of the $\mathrm{ABE}$, and the view of the Receiver can be perfectly simulated (in the $\left(\begin{array}{l}2 \\ 1\end{array}\right)$-AOT hybrid model) by outputting $\left(x, M, s, e, u, c^{\prime \prime}\right)$ where $M, s, e, u$ are sampled as in the real protocol and $c^{\prime \prime}$ is a fresh encryption of $z=a x+b$ (under the key $e$ and the matrix $M$ ).

The view of the Sender can be simulated by $\left(a, b, r, u^{\prime}, M, c\right)$ where $r, u^{\prime}$ are sampled as in the real protocol and $(M, c)$ is a fresh encryption of zero (under a uniform private key). The security of the ABE implies that the simulated view is indistinguishable from the real view.

Combined with Corollary 12.3 we derive the following corollary.

Corollary 12.8. Assuming $\operatorname{RLC}\left(n, \ell:=\frac{(1+\varepsilon) n}{1-p}, p\right)$ for some constants $p \in(0,1)$ and $\varepsilon>0$, and assuming the existence of (standard) $\left(\begin{array}{l}2 \\ 1\end{array}\right)$-OT, there exists an OLE protocol.

Remark 12.9 (Abstraction). The above construction can be abstracted by relying on any ABE which is (1) (weakly homomorphic) Given a fresh ciphertext $\operatorname{Enc}_{e}(x)$ and scalars $a, b \in \mathbb{F}$ it is possible to obtain a ciphertext $c^{\prime}$ which decrypts to $a x+b$; (2) (Secure Decryption) The "decryption" functionality $f_{\text {Dec }}$ privately reduces to $\left(\begin{array}{l}2 \\ 1\end{array}\right)$-AOT, where $f_{\text {Dec }}$ is the two-party functionality which takes a ciphertext $c^{\prime}$ from a sender and a private-key e from a receiver and delivers the value of $\operatorname{Dec}_{e}\left(c^{\prime}\right)$ to the receiver. The construction in Figure 3 follows this outline by designing a private realization for $f_{\text {Dec }}$, which is tailored to our concrete ABE. An alternative, more generic, approach can be based on information-theoretic arithmetic randomized encoding. Specifically, assume that $f_{\text {Dec }}$ admits a fully-decomposable randomized encoding (as per definition 4.6) $\hat{f}$ with a decoder $B$. Then, $f_{\text {Dec }}$ privately reduces to $\left(\begin{array}{l}2 \\ 1\end{array}\right)$-AOT via the following protocol. The ciphertext holder will choose randomness for the encoding, will send the part of the encoding $\hat{f}\left(c^{\prime}, e\right)$ that depends only on the ciphertext $c^{\prime}$ in the clear and, for the $i$-th bit of the private-key, use $\left(\begin{array}{l}2 \\ 1\end{array}\right)$-AOT to let the secret-key holder learn the part of the encoding that depends on $e_{i} \in\{0,1\}$. (See [IK00, AIK06] for a security proof of this protocol.) It is shown in [CFIK03] that any function computed by arithmetic branching program admits (information-theoretic) fully-decomposable arithmetic randomized encoding. Hence, the above approach can be applied to any weakly homomorphic ABE whose decryption algorithm can be implemented by a polynomial-size branching program.

\subsection{General Functionalities}

In this section we show in the semi-honest model a protocol for privately computing any two party functionality that is described by an arithmetic circuit over addition and multiplication gates only. We refer to such functionalities as strictly arithmetic functionalities. We do so by arithmetizing the (binary) construction of [GMW87] (see also [Gol04, Chapter 7.3]). Recall the high level structure of [GMW87]. To compute $f(x, y)$ the parties first secret share their inputs, then they propagate through the circuit that computes $f$, at each gate running a sub-procedure to compute secret shares of the output of the gate. When reaching the output wires the parties reveal the relevant secrets so that reconstruction will be possible. The main difficulty is to create a sub-procedure for computing the shares of an output of a multiplication gate. We will start by presenting such a protocol.

The two party functionality MULT takes a pair of share $\left(a_{1}, b_{1}\right) \in \mathbb{F}^{2}$ from the sender and a pair of shares $\left(a_{2}, b_{2}\right) \in \mathbb{F}^{2}$ from the receiver. (These shares correspond to the secrets $a=a_{1}+a_{2}$ and $b=b_{1}+b_{2}$.) In addition, the functionality takes a "target" share $c_{1} \in \mathbb{F}$ from the sender. The 
receiver should get a value $c_{2} \in \mathbb{F}$ that corresponds, together with $c_{1}$ to a sharing of the product $a b$. Formally, the relation $c_{1}+c_{2}=\left(a_{1}+a_{2}\right) \cdot\left(b_{1}+b_{2}\right)$ should hold ${ }^{21}$ It is not hard to privately reduce the MULT functionality to OLE.

Construction $12.10\left(\operatorname{MULT}\left(\left(a_{1}, b_{1}\right),\left(a_{2}, b_{2}\right)\right)\right)$. Consider the following 2 party protocol:

1. Inputs: Sender holds $\left(a_{1}, b_{1}, c_{1}\right)$, Receiver holds $\left(a_{2}, b_{2}\right)$.

2. Sender samples $r \stackrel{R}{\leftarrow} \mathbb{F}$.

3. The parties engage in an AOT protocol. The Sender plays the sender with inputs $\left(a_{1}, r\right)$ and the Receiver plays the receiver with the input $b_{2}$. Denote the output of the Receiver by $v_{1}$.

4. The parties engage in an AOT protocol. The Sender plays the sender with the values $\left(b_{1}, a_{1} b_{1}-\right.$ $\left.c_{1}-r\right)$ and the Receiver plays the receiver with the value $a_{2}$. Denote the output of the Receiver by $v_{2}$.

5. The Receiver outputs $c_{2}=v_{1}+v_{2}+a_{2} \cdot b_{2}$.

Lemma 12.11. Construction 12.10 privately reduces MULT to AOT.

Proof. We first observe the correctness of the protocol. Assuming the correctness of the underlying AOT, $v_{1}=a_{1} \cdot b_{2}+r$ and $v_{2}=a_{2} \cdot b_{1}+a_{1} \cdot b_{1}-c_{1}-r$ and so $c_{2}=\left(a_{1}+a_{2}\right) \cdot\left(b_{1}+b_{2}\right)-c_{1}$.

We now present the privacy reduction from Construction 12.10 to AOT. For this end we need to show a simulator for each of the parties' views when the AOT is replaced by an oracle. Such a simulation is straightforward. The Sender receives no message so the simulation is trivial. For the Receiver, the simulator gets $\left(\left(a_{2}, b_{2}\right), c_{2}=\left(a_{1}+a_{2}\right) \cdot\left(b_{1}+b_{2}\right)-c_{1}\right)$, and outputs its inputs together with $v_{1} \stackrel{R}{\leftarrow} \mathbb{F}$ and $v_{2}=c_{2}-v_{1}-a_{2} \cdot b_{2}$. It is not hard to verify that the simulated view is distributed exactly the same as the real view of the Receiver.

We now use Construction 12.10 to create our desired protocol.

Construction 12.12 (Private Circuit Evaluation). Let $C$ be an arithmetic circuit that contains only addition and multiplication gates, some of its input wires are associated with party 1 and the others with party 2. The parties execute the following protocol:

1. Secret sharing the inputs: Party 1 secret shares each its input wires in the following way: for an input wire $x$, it draws $x_{2} \stackrel{R}{\leftarrow} \mathbb{F}$ and sets $x_{1}=x-x_{2}$. It then sends $x_{2}$ to Party 2. Party 2 does the symmetric process for its input wires.

2. Emulating the circuit: Following the circuit evaluation order, the parties use their shares of the wires to compute at each gate a share of the output wire. Let $a$ and $b$ be the inputs to the gate, and $c$ the output. $\left(a_{i}, b_{i}\right)$ are party $i$ 's input and $c_{i}$-its output.

(a) Addition gates: Party $i$ locally computes $c_{i}=a_{i}+b_{i}$

(b) Multiplication gates: The parties run the multiplication sharing protocol of Construction 12.10 with the inputs $\left(a_{1}, b_{1}, c_{1}\right)$ and $\left(a_{2}, b_{2}\right)$ where $c_{1} \stackrel{R}{\leftarrow} \mathbb{F}$ and $c_{2}$ is the output of the protocol.

\footnotetext{
${ }^{21}$ This somewhat non-standard formulation allows us to work with a deterministic functionality.
} 
3. Recovering the outputs: Once the shares of the output wires are computed, each party sends its share of each output wire to the party with which the output wire is associated. Each party recovers its wires by adding the two shares for the wire.

Correctness of addition gates is straightforward, correctness of multiplication gates follows the correctness of Construction 12.10, and so the correctness of the entire protocol can be inferred by an inductive argument. We now turn to prove that the privacy of Construction 12.12 reduces to that of Construction 12.10 .

Proof sketch. Without loss of generality we describe the simulator for party 1. The simulator's inputs are all party 1's inputs: denote them by $x_{1}, \ldots, x_{n}$, and all party 1's outputs: $y_{1}, \ldots, y_{n}$. The simulator works in 3 steps:

1. Secret sharing step: Create the secret shares of party 1 as honest party 1 . For the shares sent by party 2 - sample them uniformly at random.

2. Circuit evaluation step: Evaluate addition gates as honest party 1. For multiplication gates sample the output uniformly at random.

3. Output recovery: For every output wire of party 1, the simulator was given as input the correct output value $y_{i}$. It also has a secret share of the same wire, denoted by $x_{i}$, that was computed by it in the previous steps. It outputs the second secret share for this wire as $s_{i}=y_{i}-x_{i}$.

We claim that the output of the simulation is distributed identically as the view of party 1 . The output of step 1 is identically distributed to the view of party 1 in the true execution. Conditioned on the output of step 1 , the output of step 2 is also distributed exactly as the view of party 1 , this is since the output $c_{1}$ of a multiplication gate is a uniformly random output when $c_{2}$ is excluded from the view. Finally the output generated in step 3 is a deterministic function of the previous distributions, hence does not affect the overall distribution.

Theorem 12.13. Assuming an efficient AOT there exists a protocol for privately computing any two party functionality $f$ that can be efficiently described by an arithmetic circuit with only addition and multiplication gates. In particular Construction 12.12 is such a protocol.

Combined with Corollary 12.8 , we derive the following Corollary.

Corollary 12.14. Assuming $\operatorname{RLC}\left(n, \ell:=\frac{(1+\varepsilon) n}{1-p}, p\right)$ for some constants $p \in(0,1)$ and $\varepsilon>0$, and assuming the existence of (standard) $\left(\begin{array}{l}2 \\ 1\end{array}\right)$-OT, any strictly arithmetic two-party functionality can be privately computed in the arithmetic model.

The Corollary can be easily extended to the multiparty setting by using the standard multiparty variant of Construction 12.12 .

\subsection{The Malicious Model}

In this section we briefly sketch the extension of the previous section to the malicious model.

The following theorem is implicit in [IPS09] and is based on the IPS compiler [PS08] and the arithmetic protocol for secure multiparty-computation (with information-theoretic security) of [CFIK03]. 
Theorem 12.15. Assuming the existence of semi-honest secure computation for any strictly arithmetic two-party functionality, and the existence of $\left(\begin{array}{l}2 \\ 1\end{array}\right)$-AOT with malicious security, any strictly arithmetic two-party functionality can be securely computed (with security against malicious parties) in the arithmetic model.

In Section 12.4.1 we will show that that Construction 12.1 generalizes to the malicious setting.

Theorem 12.16. Assuming that $\mathcal{E}$ is an ABE, Construction 12.1 securely reduces (in the malicious setting) $\left(\begin{array}{l}2 \\ 1\end{array}\right)$-AOT to standard binary $\left(\begin{array}{l}2 \\ 1\end{array}\right)$-OT.

Combining Theorem 12.16 with Corollary 12.14 and Corollary 10.5 we derive the following corollary.

Corollary 12.17. Assuming $\operatorname{RLC}\left(n, \ell:=\frac{(1+\varepsilon) n}{1-p}, p\right)$ for some constants $p \in(0,1)$ and $\varepsilon>0$, and assuming the existence of (standard) $\left(\begin{array}{l}2 \\ 1\end{array}\right)$-OT, any strictly arithmetic two-party functionality can be securely computed (with security against malicious parties) in the arithmetic model.

As before this can be extended to the multiparty setting using standard techniques.

\subsubsection{Proof of Theorem $\mathbf{1 2 . 1 6}$}

We reduce the malicious security of the $\left(\begin{array}{l}2 \\ 1\end{array}\right)$-AOT to the security of an Ideal $\left(\begin{array}{l}2 \\ 1\end{array}\right)$-OT functionality.

Honest Receiver. Let $A^{*}$ be any (malicious) polynomial time algorithm for the sender in the real model equipped with auxiliary input aux. We define a corresponding adversary $\operatorname{Sim}_{A^{*}}$ in the ideal model. Consider the following implementation of $\operatorname{Sim}_{A^{*}}$ (when instantiated with the field $\mathbb{F}$ and the security parameter $n$ ):

$\operatorname{Sim}_{A^{*}}\left(\operatorname{aux}, z_{0}, z_{1}\right)$ :

1. Invoke $A^{*}\left(\operatorname{aux}, z_{0}, z_{1}\right)$.

(a) Get $\mathrm{sk}_{0}^{\prime}, \mathrm{sk}_{1}^{\prime}$ that $A^{*}$ sends to the OT oracle.

(b) Get $c_{0}, c_{1}$ that $A^{*}$ sends to the receiver.

2. For $i \in\{0,1\}$ compute $z_{i}^{\prime}=\operatorname{ABDec}_{n}^{\mathbb{F}}\left(\mathrm{sk}_{i}^{\prime}, c_{i}\right)$. If decryption fails, set $z_{i}=1$.

3. Send $\left(z_{0}^{\prime}, z_{1}^{\prime}\right)$ to the trusted party and receive the empty string $\phi$ as an answer.

4. Output $A^{*}\left(\right.$ aux, $\left.z_{0}, z_{1}, \phi\right)$

It is now straightforward that for every field $\mathbb{F}$, for every input $x \in\{0,1\}, z_{0}, z_{1} \in \mathbb{F}$ and auxiliary input aux, the real and ideal distributions are identically distributed.

Honest Sender. Let $B^{*}$ be any (malicious) polynomial time algorithm for the receiver in the real model and let aux be his auxilary input. We now construct an ideal-world adversary $\operatorname{Sim}_{B^{*}}$ (for the field $\mathbb{F}$ and security parameter $n$ ):

$\operatorname{Sim}_{B^{*}}(\operatorname{aux}, x)$ :

1. Sample $\mathrm{sk}_{0}, \mathrm{sk}_{1} \stackrel{R}{\leftarrow} \mathrm{ABGen}_{n}^{\mathbb{F}}$

2. Invoke $B^{*}(\operatorname{aux}, x)$ and let $x^{\prime}$ be the request that $B^{*}$ sends to the Binary OT oracle. 
3. Send $x^{\prime}$ to the trusted party and let $z_{x^{\prime}}$ denote its answer.

4. Sample $c_{x^{\prime}}^{\prime} \stackrel{R}{\leftarrow} \mathrm{ABEnc} c_{n}^{\mathbb{F}}\left(\mathrm{sk}_{x}^{\prime}, z_{x}^{\prime}\right)$ and $c_{1-x^{\prime}}^{\prime} \stackrel{R}{\leftarrow} \mathrm{ABEnc}_{n}^{\mathbb{F}}\left(\mathrm{sk}_{1-x^{\prime}}, 0\right)$.

5. Forward $\left(\mathrm{sk}_{x^{\prime}}, c_{0}^{\prime}, c_{1}^{\prime}\right)$ to $B^{*}$ and output its output.

We will show that the simulated view is indistinguishable from the real view. Below we let $B^{*}($ aux,$x)$ denote the first message generated by $B^{*}$ and let $B^{*}\left(\right.$ aux $\left., x, x^{\prime}, \mathbf{s k}_{x}^{\prime}, c_{0}^{\prime}, c_{1}^{\prime}\right)$ denote the final output of $B^{*}$. For every field $\mathbb{F}$, and for every sequence of inputs $x \in\{0,1\}, z_{0}, z_{1} \in \mathbb{F}$, auxiliary input aux and randomness $\tau$ we have

$$
\begin{aligned}
& \left\{\operatorname{Real}_{\Pi,\left(A, B^{*}(\operatorname{aux})\right)}\left(x,\left(z_{0}, z_{1}\right)\right)\right\}_{n, x, z_{0}, z_{1}, \text { aux }} \\
& =\left\{\left(B^{*}\left(\operatorname{aux}, x, x^{\prime}, \mathrm{sk}_{x}^{\prime}, c_{0}^{\prime}, c_{1}^{\prime}\right), \phi\right), \text { where } x^{\prime} \leftarrow B^{*}(\operatorname{aux}, x)\right\}_{n, x, z_{0}, z_{1}, \text { aux }} . \\
& \begin{aligned}
c & \left\{\left(B^{*}\left(\operatorname{aux}, x, x^{\prime}, \mathrm{sk}_{x}^{\prime}, c_{0}, c_{1}\right), \phi\right), \text { where } x^{\prime} \leftarrow B^{*}(\operatorname{aux}, x)\right\}_{n, x, z_{0}, z_{1}, \text { aux }} \\
= & \left\{\operatorname{ldeal}_{f,\left(A, \operatorname{Sim}_{B^{*}(\text { aux })}\right)}\left(x,\left(z_{0}, z_{1}\right)\right)\right\}_{n, x, z_{0}, z_{1}, \text { aux }}
\end{aligned}
\end{aligned}
$$

where $c_{0}=\operatorname{ABEnc}_{n}^{\mathbb{F}}\left(\mathrm{sk}_{0}, z_{0}\right), c_{1}=\mathrm{ABEnc}_{n}^{\mathbb{F}}\left(\mathrm{sk}_{1}, z_{1}\right)$ and $\phi$ denotes the empty string. Computational indistinguishabilty follows from the security of $A B E$ scheme, since any adversary that distinguishes the two distribution immediately translates to an adversary that distinguishes an encryption of 0 from an encryption of $z_{1-x^{\prime}}$.

\section{Acknowledgement}

We thank the anonymous referees of ITCS 2015 for their useful comments, and Yuval Ishai for valuable discussions.

\section{References}

[ABX08] Benny Applebaum, Boaz Barak, and David Xiao. On basing lower-bounds for learning on worst-case assumptions. In 49th FOCS, pages 211-220. IEEE Computer Society Press, October 2008.

[ACPS09] Benny Applebaum, David Cash, Chris Peikert, and Amit Sahai. Fast cryptographic primitives and circular-secure encryption based on hard learning problems. In Shai Halevi, editor, CRYPTO 2009, volume 5677 of LNCS, pages 595-618. Springer, August 2009.

[AIK04] Benny Applebaum, Yuval Ishai, and Eyal Kushilevitz. Cryptography in $\mathrm{NC}^{0}$. In 45th FOCS, pages 166-175. IEEE Computer Society Press, October 2004.

[AIK06] Benny Applebaum, Yuval Ishai, and Eyal Kushilevitz. Computationally private randomizing polynomials and their applications. Computational Complexity, 15(2):115$162,2006$.

[AIK10] Benny Applebaum, Yuval Ishai, and Eyal Kushilevitz. Cryptography by cellular automata or how fast can complexity emerge in nature? In Andrew Chi-Chih Yao, editor, ICS 2010, pages 1-19. Tsinghua University Press, January 2010. 
[AIK11] Benny Applebaum, Yuval Ishai, and Eyal Kushilevitz. How to garble arithmetic circuits. In Rafail Ostrovsky, editor, 52nd FOCS, pages 120-129. IEEE Computer Society Press, October 2011.

[AIKW13] Benny Applebaum, Yuval Ishai, Eyal Kushilevitz, and Brent Waters. Encoding functions with constant online rate or how to compress garbled circuits keys. In Ran Canetti and Juan A. Garay, editors, CRYPTO 2013, Part II, volume 8043 of LNCS, pages 166-184. Springer, August 2013.

[Ale03] Michael Alekhnovich. More on average case vs approximation complexity. In 44th FOCS, pages 298-307. IEEE Computer Society Press, October 2003.

[AM09] Divesh Aggarwal and Ueli Maurer. Breaking RSA generically is equivalent to factoring. In Antoine Joux, editor, EUROCRYPT 2009, volume 5479 of LNCS, pages 36-53. Springer, April 2009.

[AW08] Scott Aaronson and Avi Wigderson. Algebrization: a new barrier in complexity theory. In Richard E. Ladner and Cynthia Dwork, editors, 40th ACM STOC, pages 731-740. ACM Press, May 2008.

[BDOZ11] Rikke Bendlin, Ivan Damgård, Claudio Orlandi, and Sarah Zakarias. Semihomomorphic encryption and multiparty computation. In Kenneth G. Paterson, editor, EUROCRYPT 2011, volume 6632 of LNCS, pages 169-188. Springer, May 2011.

[Bea95] Donald Beaver. Precomputing oblivious transfer. In Don Coppersmith, editor, CRYPTO'95, volume 963 of LNCS, pages 97-109. Springer, August 1995.

[BFKL93] Avrim Blum, Merrick L. Furst, Michael J. Kearns, and Richard J. Lipton. Cryptographic primitives based on hard learning problems. In Douglas R. Stinson, editor, CRYPTO'93, volume 773 of LNCS, pages 278-291. Springer, August 1993.

[BGS75] Baker, Gill, and Solovay. Relativizations of the $\mathrm{P}=$ ? NP question. SICOMP: SIAM Journal on Computing, 4, 1975.

[BHR12] Mihir Bellare, Viet Tung Hoang, and Phillip Rogaway. Foundations of garbled circuits. In Ting Yu, George Danezis, and Virgil D. Gligor, editors, ACM CCS 12, pages 784796. ACM Press, October 2012.

[BL13] Andrej Bogdanov and Chin Ho Lee. Limits of provable security for homomorphic encryption. In Ran Canetti and Juan A. Garay, editors, CRYPTO 2013, Part I, volume 8042 of LNCS, pages 111-128. Springer, August 2013.

[BM82] Manuel Blum and Silvio Micali. How to generate cryptographically strong sequences of pseudo random bits. In 23rd FOCS, pages 112-117. IEEE Computer Society Press, November 1982.

[BOGW88] Michael Ben-Or, Shafi Goldwasser, and Avi Wigderson. Completeness theorems for non-cryptographic fault-tolerant distributed computation (extended abstract). In 20th ACM STOC, pages 1-10. ACM Press, May 1988. 
[BS83] Walter Baur and Volker Strassen. The complexity of partial derivatives. Theoretical computer science, 22(3):317-330, 1983.

[BV11] Zvika Brakerski and Vinod Vaikuntanathan. Efficient fully homomorphic encryption from (standard) LWE. In Rafail Ostrovsky, editor, 52nd FOCS, pages 97-106. IEEE Computer Society Press, October 2011.

[CCD88] David Chaum, Claude Crépeau, and Ivan Damgård. Multiparty unconditionally secure protocols (extended abstract). In 20th ACM STOC, pages 11-19. ACM Press, May 1988.

[CDN01] Ronald Cramer, Ivan Damgård, and Jesper Buus Nielsen. Multiparty computation from threshold homomorphic encryption. In Birgit Pfitzmann, editor, EUROCRYPT 2001, volume 2045 of LNCS, pages 280-299. Springer, May 2001.

[CF02] Ronald Cramer and Serge Fehr. Optimal black-box secret sharing over arbitrary Abelian groups. In Moti Yung, editor, CRYPTO 2002, volume 2442 of LNCS, pages 272-287. Springer, August 2002.

[CFIK03] Ronald Cramer, Serge Fehr, Yuval Ishai, and Eyal Kushilevitz. Efficient multi-party computation over rings. In Eli Biham, editor, EUROCRYPT 2003, volume 2656 of LNCS, pages 596-613. Springer, May 2003.

[Den02] Alexander W. Dent. Adapting the weaknesses of the random oracle model to the generic group model. In Yuliang Zheng, editor, ASIACRYPT 2002, volume 2501 of LNCS, pages 100-109. Springer, December 2002.

[DF91] Yvo Desmedt and Yair Frankel. Shared generation of authenticators and signatures (extended abstract). In Joan Feigenbaum, editor, CRYPTO'91, volume 576 of LNCS, pages 457-469. Springer, August 1991.

[DGRV11] Zeev Dvir, Dan Gutfreund, Guy N. Rothblum, and Salil P. Vadhan. On approximating the entropy of polynomial mappings. In Innovations in Computer Science - ICS 2010, pages $460-475,2011$.

[DGW07] Zeev Dvir, Ariel Gabizon, and Avi Wigderson. Extractors and rank extractors for polynomial sources. In 48th FOCS, pages 52-62. IEEE Computer Society Press, October 2007 .

[DGW09] Zeev Dvir, Ariel Gabizon, and Avi Wigderson. Extractors and rank extractors for polynomial sources. Computational Complexity, 18(1):1-58, 2009.

[DORS08] Yevgeniy Dodis, Rafail Ostrovsky, Leonid Reyzin, and Adam Smith. Fuzzy extractors: How to generate strong keys from biometrics and other noisy data. SIAM J. Comput., 38(1):97-139, 2008.

[DPSZ12] Ivan Damgård, Valerio Pastro, Nigel P. Smart, and Sarah Zakarias. Multiparty computation from somewhat homomorphic encryption. In Reihaneh Safavi-Naini and Ran Canetti, editors, CRYPTO 2012, volume 7417 of LNCS, pages 643-662. Springer, August 2012. 
[ElG84] Taher ElGamal. A public key cryptosystem and a signature scheme based on discrete logarithms. In G. R. Blakley and David Chaum, editors, CRYPTO'84, volume 196 of LNCS, pages 10-18. Springer, August 1984.

[ER93] Richard Ehrenborg and Gian-Carlo Rota. Apolarity and canonical forms for homogeneous polynomials. European Journal of Combinatorics, 14(3):157-181, 1993.

[Fel07] Vitaly Feldman. Attribute-efficient and non-adaptive learning of parities and dnf expressions. Journal of Machine Learning Research, 8:1431-1460, 2007.

[FH93] Matthew K. Franklin and Stuart Haber. Joint encryption and message-efficient secure computation. In Douglas R. Stinson, editor, CRYPTO'93, volume 773 of LNCS, pages 266-277. Springer, August 1993.

[FKN94] Uriel Feige, Joe Kilian, and Moni Naor. A minimal model for secure computation (extended abstract). In 26th ACM STOC, pages 554-563. ACM Press, May 1994.

[Gen09] Craig Gentry. Fully homomorphic encryption using ideal lattices. In Michael Mitzenmacher, editor, 41st ACM STOC, pages 169-178. ACM Press, May / June 2009.

[GGH13] Sanjam Garg, Craig Gentry, and Shai Halevi. Candidate multilinear maps from ideal lattices. In Thomas Johansson and Phong Q. Nguyen, editors, EUROCRYPT 2013, volume 7881 of $L N C S$, pages 1-17. Springer, May 2013.

[GGM86] Oded Goldreich, Shafi Goldwasser, and Silvio Micali. How to construct random functions. Journal of the ACM, 33:792-807, 1986.

[GKL88] Oded Goldreich, Hugo Krawczyk, and Michael Luby. On the existence of pseudorandom generators. In Shafi Goldwasser, editor, CRYPTO'88, volume 403 of LNCS, pages 146162. Springer, August 1988.

[GL89] Oded Goldreich and Leonid A. Levin. A hard-core predicate for all one-way functions. In 21st ACM STOC, pages 25-32. ACM Press, May 1989.

[GM84] Shafi Goldwasser and Silvio Micali. Probabilistic encryption. Journal of Computer and System Sciences, 28(2):270-299, 1984.

[GMW87] Oded Goldreich, Silvio Micali, and Avi Wigderson. How to play any mental game or A completeness theorem for protocols with honest majority. In Alfred Aho, editor, 19th ACM STOC, pages 218-229. ACM Press, May 1987.

[Gol01] Oded Goldreich. Foundations of Cryptography: Basic Tools, volume 1. Cambridge University Press, Cambridge, UK, 2001.

[Gol04] Oded Goldreich. Foundations of Cryptography: Basic Applications, volume 2. Cambridge University Press, Cambridge, UK, 2004.

[GRS08] Henri Gilbert, Matthew J. B. Robshaw, and Yannick Seurin. How to encrypt with the LPN problem. In Luca Aceto, Ivan Damgård, Leslie Ann Goldberg, Magnús M. Halldórsson, Anna Ingólfsdóttir, and Igor Walukiewicz, editors, ICALP 2008, Part II, volume 5126 of LNCS, pages 679-690. Springer, July 2008. 
[GV99] Oded Goldreich and Salil P. Vadhan. Comparing entropies in statistical zero knowledge with applications to the structure of SZK. In Conference on Computational Complexity (CCC 1999), page 54, 1999.

[HILL99] Johan Håstad, Russell Impagliazzo, Leonid A. Levin, and Michael Luby. A pseudorandom generator from any one-way function. SIAM Journal on Computing, 28(4):13641396, 1999.

[IK00] Yuval Ishai and Eyal Kushilevitz. Randomizing polynomials: A new representation with applications to round-efficient secure computation. In 41st FOCS, pages 294-304. IEEE Computer Society Press, November 2000.

$\left[\mathrm{IKM}^{+} 13\right]$ Yuval Ishai, Eyal Kushilevitz, Sigurd Meldgaard, Claudio Orlandi, and Anat PaskinCherniavsky. On the power of correlated randomness in secure computation. In Amit Sahai, editor, TCC 2013, volume 7785 of LNCS, pages 600-620. Springer, March 2013.

[IKO05] Yuval Ishai, Eyal Kushilevitz, and Rafail Ostrovsky. Sufficient conditions for collisionresistant hashing. In Joe Kilian, editor, TCC 2005, volume 3378 of LNCS, pages 445-456. Springer, February 2005.

[IPS08] Yuval Ishai, Manoj Prabhakaran, and Amit Sahai. Founding cryptography on oblivious transfer - efficiently. In David Wagner, editor, CRYPTO 2008, volume 5157 of LNCS, pages 572-591. Springer, August 2008.

[IPS09] Yuval Ishai, Manoj Prabhakaran, and Amit Sahai. Secure arithmetic computation with no honest majority. In Omer Reingold, editor, TCC 2009, volume 5444 of LNCS, pages 294-314. Springer, March 2009.

[Ish12] Yuval Ishai. Randomization techniques for secure computation. In Manoj Prabhakaran and Amit Sahai, editors, Secure Multi-Party Computation, volume 10 of Cryptology and Information Security Series, pages 222-248. IOS press, Amsterdam, 2012.

[JKPT12] Abhishek Jain, Stephan Krenn, Krzysztof Pietrzak, and Aris Tentes. Commitments and efficient zero-knowledge proofs from learning parity with noise. In Xiaoyun Wang and Kazue Sako, editors, ASIACRYPT 2012, volume 7658 of LNCS, pages 663-680. Springer, December 2012.

[Kay09] Neeraj Kayal. The complexity of the annihilating polynomial. In Conference on Computational Complexity (CCC). IEEE, 2009.

[KL08] Jonathan Katz and Yehuda Lindell. Introduction to Modern Cryptography. Chapman and Hall/CRC cryptography and network security. Chapman and Hall/CRC, 2008.

$\left[\mathrm{KPC}^{+} 11\right]$ Eike Kiltz, Krzysztof Pietrzak, David Cash, Abhishek Jain, and Daniele Venturi. Efficient authentication from hard learning problems. In Kenneth G. Paterson, editor, EUROCRYPT 2011, volume 6632 of LNCS, pages 7-26. Springer, May 2011.

[Mau05] Ueli M. Maurer. Abstract models of computation in cryptography (invited paper). In Nigel P. Smart, editor, 10th IMA International Conference on Cryptography and Coding, volume 3796 of LNCS, pages 1-12. Springer, December 2005. 
[MW98] Ueli M. Maurer and Stefan Wolf. Lower bounds on generic algorithms in groups. In Kaisa Nyberg, editor, EUROCRYPT'98, volume 1403 of LNCS, pages 72-84. Springer, May / June 1998.

[Nao91] Moni Naor. Bit commitment using pseudorandomness. Journal of cryptology, 4(2):151$158,1991$.

[NP99] Moni Naor and Benny Pinkas. Oblivious transfer and polynomial evaluation. In 31st ACM STOC, pages 245-254. ACM Press, May 1999.

[Pie12] Krzysztof Pietrzak. Cryptography from learning parity with noise. In Proc. of 38th SOFSEM, pages 99-114, 2012.

[Reg05] Oded Regev. On lattices, learning with errors, random linear codes, and cryptography. In Harold N. Gabow and Ronald Fagin, editors, 37th ACM STOC, pages 84-93. ACM Press, May 2005.

[RR94] Alexander A. Razborov and Steven Rudich. Natural proofs. In 26th ACM STOC, pages 204-213. ACM Press, May 1994.

[Sch80] Jacob T Schwartz. Fast probabilistic algorithms for verification of polynomial identities. Journal of the ACM (JACM), 27(4):701-717, 1980.

[Sha79] Adi Shamir. How to share a secret. Communications of the Association for Computing Machinery, 22(11):612-613, November 1979.

[Sho97] Victor Shoup. Lower bounds for discrete logarithms and related problems. In Walter Fumy, editor, EUROCRYPT'97, volume 1233 of LNCS, pages 256-266. Springer, May 1997.

[SY10] Amir Shpilka and Amir Yehudayoff. Arithmetic circuits: A survey of recent results and open questions. Foundations and Trends in Theoretical Computer Science, 5(34):207-388, 2010.

[VGS14] Atri Rudra Venkatesan Guruswami and Madhu Sudan. Essential Coding Theory. 2014. http://www.cse.buffalo.edu/ atri/courses/coding-theory/book/,

[Yao82] Andrew Chi-Chih Yao. Theory and applications of trapdoor functions (extended abstract). In 23rd FOCS, pages 80-91. IEEE Computer Society Press, November 1982.

[Yao86] Andrew Chi-Chih Yao. How to generate and exchange secrets (extended abstract). In 27th FOCS, pages 162-167. IEEE Computer Society Press, October 1986.

[Zip79] Richard Zippel. Probabilistic algorithms for sparse polynomials. Springer, 1979. 